\title{
Statistics of SPDEs: From Linear to Nonlinear
}

\author{
Jaya P. N. Bishwal \\ Department of Mathematics and Statistics, University of North Carolina at Charlotte, \\ 376 Fretwell Bldg, 9201 University City Blvd. Charlotte, NC 28223-0001, USA \\ Correspondence: J.Bishwal@uncc.edu
}

\begin{abstract}
We study statistical inference for stochastic partial differential equations (SPDEs). Though inference linear SPDEs have been studied well (with lot of problems still remain to be investigated) in the last two decades, inference for nonlinear SPDEs is in its infancy. The inference methods use both inference for finite-dimensional diffusions and inference for classical i.i.d. sequences. Solving 2D Navier-Stokes equation is one of the challenging problem of the last century. However, with additive white noise, the equation has a strong solution. We estimate the viscosity coefficient of the 2D stochastic Navier-Stokes (SNS) equation by minimum contrast method. We show $n$ consistency in contrast to $\sqrt{n}$ consistency in the classical i.i.d. case where $n$ is the number of observations. We consider both continuous and discrete observations in time. We also obtain the Berry-Esseen bounds. Then we estimate and control the Type I and Type II error of a simple hypothesis testing problem of the viscosity coefficient of the SNS equation. We study a class of rejection regions and provide thresholds that guarantee that the statistical errors are smaller than the given upper bound. The tests are of likelihood ratio type. The proofs are based on the large deviation bounds. Finally we give Monte Carlo test procedure for simulated data.
\end{abstract}

\section{Introduction and Preliminaries}

Recently infinite dimensional stochastic differential equations (SDEs), such as the stochastic partial differential equations (SPDEs) are being paid a lot of attention in view of their modeling applications in cell biology, neurophysiology, turbulence and oceonography and finance: see Itô (1984), Walsh (1986), Kallianpur and Xiong (1995), Holden et al. (1996), Adler et al. (1996), Carmona and Rozovskii (1999) and Bishwal (2017).

Parameter estimation is an inverse problem. Loges (1984) initiated the study of parameter estimation in infinite dimensional stochastic differential equations. When the length of the observation time becomes large, he obtained consistency and asymptotic normality of the maximum likelihood

Received: 19 Sep 2021.

Key words and phrases. nonlinear SPDE; Navier-Stokes equation; additive white noise; viscosity; coupling; Galerkin method; minimum contrast estimator; Berry-Esseen inequality; hypothesis testing; Monte Carlo simulation. 
estimator (MLE) of a real valued drift parameter in a Hilbert space valued SDE. Koski and Loges (1986) extended the work of Loges (1984) to minimum contrast estimators. Koski and Loges (1985) applied the work to a stochastic heat flow problem. Mohapl (1992) studied the asymptotics of MLE in a in a nuclear space valued SDE. Kim (1996) also studied the properties of MLE in a similar set up. See the monograph Bishwal (2008) for recent results on likelihood asymptotics and Bayesian asymptotics for drift estimation of finite and infinite dimensional stochastic differential equations.

Huebner and Rozovskii (1995). introduced spectral method to study consistency, asymptotic normality and asymptotic efficiency of MLE of a parameter in the drift coefficient of an SPDE. This approach allows one to obtain asymptotics of estimators under conditions which guarantee the singularity of the measures generated by the corresponding diffusion field for different parameters. Unlike in the finite dimensional cases, where the total observation time was assumed to be long $(T \rightarrow \infty)$ or intensity of the noise was assumed to be small $(\epsilon \rightarrow 0)$, here both are kept fixed. Here the asymptotics are obtained when the number of Fourier coefficients $(n)$ of the solution of SPDE becomes large.

Huebner, Khasminskii and Rozovskii (1992) started statistical investigation in SPDEs. They gave two contrast examples of parabolic SPDEs in one of which they obtained consistency, asymptotic normality and asymptotic efficiency of the MLE as noise intensity decreases to zero under the condition of absolute continuity of measures generated by the process for different parameters (the situation is similar to the classical finite dimensional case) and in the other they obtained these properties as the finite dimensional projection becomes large under the condition of singularity of the measures generated by the process for different parameters. The second example was extended by Huebner and Rozovskii (1995) and the first example was extended by Huebner (1999) to MLE for general parabolic SPDEs where the partial differential operators commute and satisfy different order conditions in the two cases.

For partially observed SPDE systems of both parabolic and hyperbolic type, parameter estimation is studied by Aihara (1992, 1994, 1995), Aihara and Bagchi (1988, 1989, 1991), Bagchi and Borkar (1984). The asymptotics when the finite dimensional approximations to solutions of SPDEs becomes large was studied by Huebner and Rozovskii (1995).

Under some conditions they showed that the MLE of the drift parameter in parabolic SPDE is consistent, asymptotically normal and asymptotically efficient as $n \rightarrow \infty$. The spectral asymptotics for MLE was exteded by Huebner and Rozovskii (1995) (here after HR) to more general SPDEs where the partial differential operators commute and satisfy some order conditions. Piterberg and Rozovskii (1995) studied the properties MLE of a parameter in SPDE which are used to model the upper oceon variability in physical oceonogoaphy. Piterbarg and Rozovskii (1996) studied the properties of MLE based on discrete observations of the corresponding diffusion field. 
Huebner (1997) extended the problem to the ML estimation of multidimensional parameter. Lototsky and Rozovskii (1999) studied the same problem without the commutativity condition. Small noise asymptotics of the nonparmetric estimation of the drift coefficient was studies by lbragimov and Khasminskii (1998).

While moving from linear to nonlinear equation, stochastic Navier-Stokes equation in both 2 and 3 dimensions have been extensively studied. See for instance, Lions and Magenes (1972), Bensoussan and Temam (1973), Breckner (2000), Mikelevicius and Rozovskii (2004). However parameter estimation for nonlinear equation is fairly new.

Consider the 2D stochastic Navier-Stokes equation

$$
\begin{aligned}
& d U_{t}=(\theta \Delta U-(U \cdot \nabla) U-\nabla P) d t+d W_{t} \\
& \nabla \cdot U=0 \\
& U_{0}=\xi
\end{aligned}
$$

which describe the flow of a viscous incompressible fluid. Here $U=\left(U_{1}, U_{2}\right)$ is the velocity and $P$ is the pressure.

The coefficient $\theta>0$ corresponds to the kinematic viscosity of the fluid which is the unknown parameter to be estimated. Let $u_{k}=\left(U, \phi_{k}\right)$ be the $k$-th generalized Fourier mode of the solution $U$. Each Fourier mode $u_{k}$ represents a one-dimensional stable Ornstein-Uhlenbeck process.

We estimate the parameter and test hypothesis based on a sample path $U(\omega)$ observed over a finite time interval $[0, T]$. We assume that the governing equation evolve over a domain $D$. We will consider two possible boundary conditions. In the first case we suppose that the flow occurs over all of $\mathbb{R}^{2}$ with $D=[-L / 2, L / 2]^{2}$ for some $L>0$ and prescribe the periodic boundary condition:

$$
U\left(x+L e_{j}, t\right)=U(x, t), x \in \mathbb{R}^{2}, t \geq 0 ; \int_{D} U(x) d x=0 .
$$

We will also consider the case when $D$ is a bounded subset of $\mathbb{R}^{2}$ with a smooth boundary $\partial D$ and assume the Dirichlet (no slip) boundary condition:

$$
U(x, t)=0, x \in \partial D, t \geq 0 .
$$

The stochastic forcing we consider is an additive space-time noise colored in space. Formally we may write

$$
\sigma d W=\sum_{k} \lambda_{k}^{-\gamma} \phi_{k} d W_{k}
$$

where $\phi_{k}$ are eigenfunctions of the Stokes operator, $\lambda_{k}$ represents the associated eigenvalues and $W_{k}, k \geq 1$ are one-dimensional independent Brownian motions. We assume $\gamma$ is a real parameter greater than 1 which guarantees some spatial smoothness in the forcing. We may also derive the space-time correlation structure of the noise term

$$
E(\sigma d W(x, t) \sigma d W(y, s))=K(x, y) \delta_{t-s}
$$


where

$$
K(x, y)=\sum_{k \geq 1} \lambda_{k}^{-2 \gamma} \phi_{k}(x) \phi_{k}(y) .
$$

The stochastic Stokes equation associated with the SNE is

$$
d \bar{U}=-\theta A \bar{U} d t+\sum_{k} \lambda_{k}^{-\gamma} \phi_{k} d W_{k}, \bar{U}(0)=\bar{U}_{0}
$$

This is a $2 \mathrm{D}$ stochastic heat equation driven by additive cylindrical Brownian motion.

Let us denote $\bar{u}_{k}, k \geq 1$, the Fourier coefficient of the solution $\bar{U}$ with respect to the system $\{\phi\}_{k}$ in $H$, i.e., $\bar{u}_{k}=\left(\bar{U}, \phi_{k}\right), k \geq 1$. By (1.8), each Fourier mode represents a one dimensional stable OU process with dynamics

$$
d \bar{u}_{k}+\theta \lambda_{k} \bar{u}_{k} d t=\lambda_{k}^{-\gamma} d W_{k}, \bar{u}_{k}(0)=\bar{u}_{0 k}, k \geq 1
$$

The solution of this equation is

$$
\bar{u}_{k}(t)=\bar{u}_{k}(0) e^{-\theta \lambda_{k} t}+\lambda_{k}^{-\gamma} \int_{0}^{t} e^{-\theta \lambda_{k}(t-s)} d W_{k}(s), \quad k \geq 1, \quad t \geq 0 .
$$

Here $\bar{u}_{k}=\left(\bar{U}, \phi_{k}\right)$ represents the $k$-th generalized Fourier mode of the solution $U$.

This approximation may be rigourously justified by Galerkin approximation. We project $U$ down to a finite dimensional space and for each $n, U^{n}=P_{n} U, \quad n \geq 1$ where $P_{n}$ is the projection operator on the finite dimensional space generated by the first $n$ Fourier eigenvalues of the Stokes operator. The $U^{n}$ satisfies

$$
d U^{n}=-\left(\theta A U^{n}+P_{n}(B(U)) d t+\sigma P_{n} d W, U^{n}(0)=U_{0}^{n}\right.
$$

Though the main results in this work are similar to the previous works in the linear case, the difficulties arise here due to the complex nature of the nonlinear term which couples all the modes of $u_{k}=\left(U, \phi_{k}\right)$. In contrast to the linear case, we lose the explicit spectral information about the elements $u_{k}$ because of coupling, $u_{k}$ are not independent. To overcome this difficulty, we decompose, $U=\bar{U}+R$. Here $\bar{U}$ is a linear system where the Fourier modes are independent.

Recast (1.1) as a stochastic evolution equation

$$
\begin{aligned}
& d U=-(\theta A U+B(U)) d t+\sigma d W \\
& U(0)=U_{0} .
\end{aligned}
$$

We now introduce the basic function spaces designed to capture both the boundary conditions and the divergence free nature of the flow.

We first consider the space associated with the Dirichlet (no slip) boundary condition (1.3). Let $H:=\left\{U \in L^{2}(D)^{2}: \nabla \cdot U=0, U \cdot n=0\right\}$ where $n$ is the outer pointing unit normal to $\partial D$. The space $H$ is endowed as a Hilbert space with the $L^{2}$ inner product $\left(U^{b}, U^{c}\right)=\int_{D} U^{b} U^{c} d x$ and the associated norm $|U|=(U \cdot U)^{1 / 2}$. The Leray-Hopf projector $P_{H}$ is defined as the orthogonal projection of $L^{2}(D)^{d}$ onto $H$. Let $V:=\left\{U \in H_{0}^{1}(D)^{2}: \nabla \cdot U=0\right\}$ and endow this with the inner 
product $\left(U^{b}, U^{c}\right)=\int_{M} \nabla U^{b} \cdot \nabla U^{c} d x$. Due to the Dirichlet boundary conditions (1.3), the Poincare inequality $|U| \leq c\|U\|$ holds for $U \in V$ justifying the definition.

Suppose the periodic boundary condition (1.2) holds. We take $D=[-L / 2, L / 2]^{2}$ and define the spaces $L_{p e r}^{2}(D)^{2}, H_{p e r}^{1}(D)^{2}$ to be the family of vector fields $U=U(x)$ which are $L$ periodic in each direction and which belongs to $L^{2}(G)^{2}$ and $H^{1}(G)^{2}$ respectively for every open bounded set $G \subset \mathbb{R}^{2}$. We define

$$
H=\left\{U \in L_{p e r}^{2}(D)^{2}: \nabla \cdot U=0, \int_{D} U(x) d x=0\right\}
$$

and

$$
V=\left\{U \in H_{p e r}^{1}(D)^{2}: \nabla \cdot U=0, \int_{D} U(x) d x=0\right\}
$$

The spaces $H$ and $V$ are endowed with the norms $|\cdot|$ and $\|\cdot\|$ respectively as above. We impose the mean zero condition for defining $H$ and $V$ so that the Poincare inequality holds (cf. Temam (1995)).

The linear portion of the SNS equation (1.1) is captured by the Stokes operator $A=-P_{H} \Delta$ which is an unbounded operator from $H$ to $H$ with domain $D(A)=H^{2}(M) \cap V$. Since $A$ is self adjoint with compact inverse $A^{-1}: H \rightarrow D(A)$, we may apply the standard theory of compact, symmetric operators to guarantee the existence of an orthonormal basis $\left\{\phi_{k}, k \geq 1\right\}$ for $H$ of eigen functions of $A$ with the associated eigenvalues $\left\{\lambda_{k}, k \geq 1\right\}$ forming an unbounded increasing sequence. Moreover,

$$
\frac{\lambda_{k}}{k \lambda_{1}} \rightarrow 1 \text { as } k \rightarrow \infty
$$

For more details on the asymptotic behavior of the sequence $\left\{\lambda_{k}, k \geq 1\right\}$, see Babenko (1982) and Metivier (1978) for the no slip case (1.3) and Constantin and Foias (1988) for the periodic case (1.2).

Define $H_{n}=\operatorname{Span}\left\{\phi_{1}, \ldots, \phi_{n}\right\}$ and let $P_{n}$ be the projection from $H$ on to this space. We let $Q_{n}:=1-P_{n}$.

Given $\alpha>0$, let $D\left(A^{\alpha}\right)=\left\{U \in H: \sum_{k} \lambda_{k}^{2 \alpha}\left|u_{k}\right|^{2}<\infty\right\}$, where $u_{k}=\left(U, \phi_{k}\right)$. For $U \in D\left(A^{\alpha}\right)$, define

$$
A^{\alpha}(U)=\sum_{k} \lambda_{k}^{\alpha} u_{k} \phi_{k}
$$

for $U=\sum_{k} u_{k} \phi_{k}$. Note that

$$
\left|A^{\alpha_{2}} P_{n} U\right| \leq \lambda_{n}^{\alpha_{2}-\alpha_{1}}\left|A^{\alpha_{1}} P_{n} U\right|
$$

and

$$
\left|A^{\alpha_{1}} Q_{n} U\right| \leq \lambda_{n}^{\alpha_{1}-\alpha_{2}}\left|A^{\alpha_{2}} Q_{n} U\right|
$$

for any $\alpha_{1}<\alpha_{2}$.

Now we consider the stochastic terms in (1.1). Let $\mathcal{S}=\left(\Omega, \mathcal{F}, P,\{\mathcal{F}\}_{t \geq 0},\left\{W_{k}\right\}_{k \geq 1}\right)$ be a stochastic basis which is a complete filtered probability space with $\left\{W_{k}, k \geq 1\right\}$, a sequence of 
independent standard Brownian motions relative to the filtration $\left\{\mathcal{F}_{t}\right\}_{t \geq 0}$. We assume $\mathcal{F}_{t}$ to be complete and continuous. Writing formally $W=\sum_{k \geq 0} \phi_{k} W_{k}, W$ may be viewed as a cylindrical Brownian motion on $H$.

We consider the family of Hilbert-Schmidt operators mapping $H$ into $D\left(A^{\beta}\right), \beta \geq 0$. We denote the family by $L_{2}\left(H, D\left(A^{\beta}\right)\right)$. We assume that $\sigma$, understood as an operator, has the form

$$
\sigma \phi_{k}=\lambda_{k}^{-\gamma} \phi_{k}
$$

and we write

$$
\sigma d W(t)=\sum_{k=1}^{\infty} \lambda_{k}^{-\gamma} \phi_{k} d W_{k}(t), \quad t \geq 0 .
$$

Given the assumption that $\gamma>1$, we have $\sigma \in L_{2}\left(H, D\left(A^{1 / 2}\right)\right)$.

We may also formally derive the space-time corelation function structure of the noise term

$$
E(\sigma d W(x, t) \sigma d W(y, s))=K(x, y) \delta_{t-s}
$$

where $K(x, y)=\sum_{k \geq 1} \lambda_{k}^{-2 \gamma} \phi_{k}(x) \phi_{k}(y)$.

\section{Stochastic Stokes Equation}

We do not have a precise spectral information about the Fourier coefficients $u_{k}=\left(U, \phi_{k}\right), k \geq 1$ in contrast to the linear case. To overcome this, we proceed to decompose the solution into a linear and nonlinear part $U=\bar{U}+R$. The linear system associated with SNS equation (1.1) is the stochastic Stokes equation. The linear term $\bar{U}$ satisfies

$$
d \bar{U}+\theta A \bar{U} d t=\sum_{k} \lambda_{k}^{-\gamma} \phi_{k} d W_{k}, \quad \bar{U}(0)=\bar{U}_{0} .
$$

This system can be analyzed as a 2D stochastic heat equation driven by an additive cylindrical Brownian motion.

\section{Nonlinear Terms}

The residual $R$ satisfies

$$
\partial_{t} R+\theta A R=-B(U), \quad R(0)=R_{0} .
$$

The regularity properties of the residual $R$ shows that $R$ is slightly smoother than $\bar{U}$. This extra regularity properties of $R$ is used to show that the nonlinear part in the MLE, namely

$$
\frac{\int_{0}^{T}\left(A U^{n}\right)^{\prime} G^{2} P_{n}(B(U)) d t}{\int_{0}^{T}\left(A U^{n}\right)^{\prime} G^{2}\left(A U^{n}\right) d t}
$$

converges to zero as $n \rightarrow \infty$. 
Existence and Uniqueness of a strong pathwise solution of SNS Equation

We recall now some well established existence, uniqueness and regularity results for these equations. The solutions we consider corresponds to 'strong solutions' in the deterministic setting, see Temam (2001). In our case since the stochastic basis $\mathcal{S}$ is fixed in advance, the considered solution is 'strong' (or pathwise) in the probabilistic sense.

Lemma 1.1 (Cialenko and Glatt-Holtz (2011))

i) Suppose we impose the periodic boundary conditions (1.2) or the Dirichlet boundary conditions (1.3) and assume that $U_{0} \in V, \sigma \in L_{2}(H, V)$. Then there exists a unique, $H$-valued, $\mathcal{F}_{t}$-adapted process $U$ with

$$
U \in L_{\text {loc }}^{2}([0, \infty) ; D(A)) \cap C([0, \infty) ; V) \text { a.s. }
$$

and so that for each $t \geq 0$,

$$
U_{t}+\int_{0}^{t}(\theta A U+B(U)) d u=U_{0}+\sum_{k} \sigma \phi_{k} W_{k}(t)
$$

with the equality understood in $\mathrm{H}$.

ii) In the case of periodic boundary conditions (1.2), if $\beta>1 / 2$ so that $\sigma \in L_{2}\left(H, D\left(A^{\beta}\right)\right), U_{0} \in$ $D\left(A^{\beta}\right)$, then

$$
U \in L_{\text {loc }}^{2}\left([0, \infty) ; D\left(A^{\beta+1 / 2}\right)\right) \cap C\left([0, \infty) ; D\left(A^{\beta}\right)\right)
$$

We need the following preliminary results on LLN and CLT to prove our main theorems.

Lemma 1.2 (Law of Large Numbers)

Let $\xi_{n}, n \geq 1$ be a sequence of random variables and $b_{n}, n \geq 1$ be an increasing sequence of positive numbers such that $\lim _{n \rightarrow \infty} b_{n}=\infty$ and

$$
\sum_{n=1}^{\infty} \frac{\operatorname{Var}\left(\xi_{n}\right)}{b_{n}^{2}}<\infty
$$

i) If the random variables $\xi_{n}$ are independent then,

$$
\lim _{n \rightarrow \infty} \frac{\sum_{k=1}^{n}\left(\xi_{k}-E \xi_{k}\right)}{b_{n}}=0 \text { a.s. }
$$

ii) If the random variables $\xi_{n}$ are uncorrelated then,

$$
\lim _{n \rightarrow \infty} \frac{\sum_{k=1}^{n}\left(\xi_{k}-E \xi_{k}\right)}{b_{n}}=0 \text { in probability. }
$$

Part (i) is from Shiryayev (1996). Part (ii) can be proved by using Markov inequality. 
Lemma 1.3 (CLT for Stochastic Integrals)

Let $\mathcal{S}=\left(\Omega, \mathcal{F}, P,\{\mathcal{F}\}_{t \geq 0},\left\{W_{k}\right\}_{k \geq 1}\right)$ be a stochastic basis. Suppose that $\sigma_{k} \in L^{2}\left(\Omega ; L^{2}([0, T])\right)$ be a sequence of real valued predictable processes such that

Then

$$
\frac{\sum_{k=1}^{n} \int_{0}^{T} \sigma_{k} d W_{k}(t)}{\left(\sum_{k=1}^{n} E \int_{0}^{T} \sigma_{k}^{2} d t\right)^{1 / 2}} \rightarrow 1 \text { in probability as } n \rightarrow \infty
$$

\section{Parameter Estimation from Continuous Observation}

\subsection{First Order Asymptotic Theory}

We study the consistency and asymptotic normality of the minimum contrast estimator in this subsection. The $n$ Fourier modes $u_{k}(t), k=1,2, \ldots, n, 0 \leq t \leq T$ are the observations. Let $U^{n}$ be the projection of the solution of the solution $U$ onto $H_{n}=P_{n} H \approx \mathbb{R}^{n}$. The finite dimensional system is given by

$$
d U^{n}=-\left(\theta A U^{n}+P_{n}(B(U)) d t+\sigma P_{n} d W, U^{n}(0)=U_{0}^{n}\right.
$$

Let $P_{\theta}^{n, T}$ be the probability measure on $C\left([0, T] ; \mathbb{R}^{n}\right)$ generated by $U^{n}$. Let $\theta_{0}$ be the true value of the parameter $\theta$. Then the Radon-Nikodym derivative or likelihood ratio $\frac{d \mathbb{P}_{\theta}^{n, T}}{d \mathbb{P}_{\theta_{0}}^{n, T}}\left(U^{n}\right)$ is given by

$$
\begin{aligned}
\frac{d \mathbb{P}_{\theta}^{n, T}}{d \mathbb{P}_{\theta_{0}}^{n, T}}\left(U^{n}\right)= & \exp \left(-\left(\theta-\theta_{0}\right) \int_{0}^{T}\left(A U^{n}\right)^{\prime} G^{2} d U^{n}-\frac{1}{2}\left(\theta^{2}-\theta_{0}^{2}\right) \int_{0}^{T}\left(A U^{n}\right)^{\prime} G^{2} A U^{n} d t\right. \\
& \left.-\left(\theta-\theta_{0}\right) \int_{0}^{T}\left(A U^{n}\right)^{\prime} G^{2}\left(A U^{n}\right) \psi_{n} d t\right)
\end{aligned}
$$

where $G:=\left(P_{n} \sigma\right)^{-1}, \psi_{n}:=P_{n}(B(U))$. By maximizing the likelihood ratio with respect to the parameter of interest $\theta$, one obtains the maximum likelihood estimator (MLE) of $\theta$ which is given by

$$
\breve{\theta}_{n, T}=-\frac{\int_{0}^{T}\left(A U^{n}\right)^{\prime} G^{2} d U^{n}+\int_{0}^{T}\left(A U^{n}\right)^{\prime} G^{2} P_{n}(B(U)) d t}{\int_{0}^{T}\left(A U^{n}\right)^{\prime} G^{2}\left(A U^{n}\right) d t} .
$$

Action of $G^{2}$ on $H_{n}$ is equivalent to $A^{2 \gamma}$.

A modified MLE is given by

$$
\bar{\theta}_{n, T}=-\frac{\int_{0}^{T}\left\langle A^{1+2 \alpha} U^{n}, d U^{n}\right\rangle+\int_{0}^{T}\left\langle A^{1+2 \alpha} U^{n}, P_{n}(B(U))\right\rangle d t}{\int_{0}^{T}\left|A^{1+\alpha} U^{n}\right|^{2} d t}
$$


where $\alpha$ is a free parameter with a range specified later on. The action $G^{2}$ on $H_{n}$ s equivalent to $A^{2 \gamma}$. Also observe that $\bar{\theta}_{n, T}$ is a special case of $\check{\theta}_{n, T}$ with $\alpha=\gamma$. Although $\bar{\theta}_{n, T}$ has the desirable theoretical properties, it also assumes that $P_{n}(B(U)$ is computable which could be a difficult task. Another estimator is obtained by approximation: By replacing $P_{n}\left(B(U)\right.$ with $P_{n}\left(B\left(U^{n}\right)\right)$

$$
\begin{gathered}
\overline{\bar{\theta}}_{n, T}=-\frac{\int_{0}^{T}\left\langle A^{1+2 \alpha} U^{n}, d U^{n}\right\rangle+\int_{0}^{T}\left\langle A^{1+2 \alpha} U^{n}, P_{n}\left(B\left(U^{n}\right)\right)\right\rangle d t}{\int_{0}^{T}\left|A^{1+\alpha} U^{n}\right|^{2} d t} \\
=-\frac{\int_{0}^{T}\left\langle A^{1+2 \alpha} U^{n}, d U^{n}\right\rangle}{\int_{0}^{T}\left|A^{1+\alpha} U^{n}\right|^{2} d t}-\frac{\int_{0}^{T}\left\langle A^{1+2 \alpha} U^{n}, P_{n}\left(B\left(U^{n}\right)\right)\right\rangle d t}{\int_{0}^{T}\left|A^{1+\alpha} U^{n}\right|^{2} d t}=: \tilde{\theta}_{n, T}+\kappa_{n, T}
\end{gathered}
$$

which depends on the first $n$ Fourier modes.

The term

$$
\kappa_{n, T}:=-\frac{\int_{0}^{T}\left\langle A^{1+2 \alpha} U^{n}, P_{n}\left(B\left(U^{n}\right)\right)\right\rangle d t}{\int_{0}^{T}\left|A^{1+\alpha} U^{n}\right|^{2} d t}
$$

is lower order and tends to zero as $n \rightarrow \infty$, see Corollary 2.2.

The linearized MLE is given by

$$
\begin{aligned}
\hat{\theta}_{n, T} & =-\frac{\int_{0}^{T}\left\langle A^{1+2 \alpha} U^{n}, d U^{n}\right\rangle}{\int_{0}^{T}\left|A^{1+\alpha} U^{n}\right|^{2} d t}=\frac{\sum_{k=1}^{n} \lambda_{k}^{1+2 \alpha} \int_{0}^{T} u_{k} d u_{k}}{\sum_{k=1}^{n} \lambda_{k}^{2+2 \alpha} \int_{0}^{T} u_{k}^{2} d t} \\
& =\frac{\sum_{k=1}^{n} \lambda_{k}^{1+2 \alpha}\left(u_{k}^{2}(T)-u_{k}^{2}(0)-T \lambda_{k}^{-2 \gamma}\right)}{2 \sum_{k=1}^{n} \lambda_{k}^{2+2 \alpha} \int_{0}^{T} u_{k}^{2} d t} .
\end{aligned}
$$

Following Bishwal (2006), we propose the minimum contrast estimator (MCE) of $\theta$ which is given by

$$
\tilde{\theta}_{n, T}=\frac{-T \sum_{k=1}^{n} \lambda_{k}^{1+2 \alpha-2 \gamma}}{2 \sum_{k=1}^{n} \lambda_{k}^{2+2 \alpha} \int_{0}^{T} u_{k}^{2} d t} .
$$

Remark 2.1 It is known that MCE is asymptotically efficient but OLSE is not efficient for the finite dimensional OU process, see Tanaka (2013).

The following two propositions are from Cialenko and Glatt-Holtz (2011).

\section{Proposition 2.1}

a) For every $\delta_{1}<\min \{2+2 \alpha-2 \gamma, 1\}$ we have

$$
n^{\delta_{1}} \frac{\int_{0}^{T}\left\langle A^{1+2 \alpha-\gamma} \bar{U}^{n}, \sum_{k} \phi_{k} d W^{k}\right\rangle}{\int_{0}^{T}\left|A^{1+\alpha} U^{n}\right|^{2} d t} \rightarrow^{\text {a.s. }} 0 \text { as } n \rightarrow \infty .
$$

b) Whenever $\delta_{2}<\min \{2+2 \alpha-2 \gamma, 3 / 2\}$ in the case of periodic boundary conditions (1.2) or whenever $\delta_{2}<\min \{2+2 \alpha-2 \gamma, 5 / 4+1-\gamma\}$ in the case of Dirichlet boundary conditions (1.3), we have

$$
n^{\delta_{2}} \frac{\int_{0}^{T}\left\langle A^{1+2 \alpha-\gamma} R^{n}, \sum_{k} \phi_{k} d W^{k}\right\rangle}{\int_{0}^{T}\left|A^{1+\alpha} U^{n}\right|^{2} d t} \rightarrow^{P} 0 \text { as } n \rightarrow \infty
$$


Corollary 2.1 Putting $\delta_{1}=\delta_{2}=0$ and noting that $U^{n}=\bar{U}^{n}+R^{n}$, we have

$$
\frac{\int_{0}^{T}\left\langle A^{1+2 \alpha-\gamma} U^{n}, \sum_{k} \phi_{k} d W^{k}\right\rangle}{\int_{0}^{T}\left|A^{1+\alpha} U^{n}\right|^{2} d t} \rightarrow^{P} 0 \text { as } n \rightarrow \infty .
$$

The following proposition is about the nonlinear term.

\section{Proposition 2.2}

For every $\delta \in[0, \min \{1 / 2, \alpha-\gamma+1\}$ in the case (1.2) or $\delta \in[0, \min \{5 / 4-\gamma, \alpha-\gamma+1\}$ in the case of Dirichlet boundary condition (1.3), we have

$$
n^{\delta} \frac{\int_{0}^{T}\left\langle A^{1+2 \alpha} U^{n}, P_{n} B(U)\right\rangle d t}{\int_{0}^{T}\left|A^{1+\alpha} U^{n}\right|^{2} d t} \rightarrow 0 \text { a.s. as } n \rightarrow \infty .
$$

Corollary 2.2 Putting $\delta=0$,

$$
\frac{\int_{0}^{T}\left\langle A^{1+2 \alpha} U^{n}, P_{n} B(U)\right\rangle d t}{\int_{0}^{T}\left|A^{1+\alpha} U^{n}\right|^{2} d t} \rightarrow 0 \text { a.s. as } n \rightarrow \infty .
$$

Thus $\kappa_{n, T} \rightarrow 0$ a.s. as $n \rightarrow \infty$..

The following is the main result of this sub-section.

\section{Theorem 2.1}

a) If $\alpha>\gamma-1$, then $\lim _{n \rightarrow \infty} \hat{\theta}_{n, T}=\theta$ in probability as $n \rightarrow \infty$.

b) If $\alpha>\gamma-1 / 2$, then $n\left(\hat{\theta}_{n, T}-\theta\right) \rightarrow^{D} \eta$ as $n \rightarrow \infty$

where $\eta$ is a normal random variable with mean zero and variance $\frac{2 \theta(\alpha-\gamma+1)^{2}}{\lambda_{1} T(\alpha-\gamma+1 / 2)}$.

\section{Classical Proof:}

a) Consistency

We have for the linearized MLE

$$
\begin{aligned}
& \hat{\theta}_{n, T}=\theta-\frac{\int_{0}^{T}\left\langle A^{1+2 \alpha-\gamma} U^{n}, P_{n} \sigma d W\right\rangle}{\left.\int_{0}^{T}\left|A^{1+\alpha} U^{n}\right|^{2} d t\right)} \\
& =\theta-\frac{\int_{0}^{T}\left\langle A^{1+2 \alpha-\gamma} U^{n}, \sum_{k} \phi_{k} d W^{k}\right\rangle}{\int_{0}^{T}\left|A^{1+\alpha} U^{n}\right|^{2} d t} .
\end{aligned}
$$

Similarly for the MLE

$$
\breve{\theta}_{n, T}=\theta-\frac{\int_{0}^{T}\left\langle A^{1+2 \alpha-\gamma} U^{n}, \sum_{k} \phi_{k} d W^{k}\right\rangle}{\int_{0}^{T}\left|A^{1+\alpha} U^{n}\right|^{2} d t}
$$


Since $\tilde{\theta}_{n, T}=\hat{\theta}_{n, T}-\kappa_{n, T}$, where

$$
+\frac{\int_{0}^{T}\left\langle A^{1+2 \alpha-\gamma} U^{n}, P_{n} B(U)-P_{n} B\left(U^{n}\right)\right\rangle d t}{\int_{0}^{T}\left|A^{1+\alpha} U^{n}\right|^{2} d t} .
$$

$$
\kappa_{n}=-\frac{\int_{0}^{T}\left\langle A^{1+2 \alpha-\gamma} U^{n}, P_{n} B(U)\right\rangle d t}{\int_{0}^{T}\left|A^{1+\alpha} U^{n}\right|^{2} d t},
$$

we have

$$
\hat{\theta}_{n}=\theta-\kappa_{n}-\frac{\int_{0}^{T}\left\langle A^{1+2 \alpha-\gamma} U^{n}, \sum_{k} \phi_{k} d W^{k}\right\rangle}{\int_{0}^{T}\left|A^{1+\alpha} U^{n}\right|^{2} d t} .
$$

We have for the MCE

$$
\begin{aligned}
& \tilde{\theta}_{n, T}=\theta-\frac{\int_{0}^{T}\left\langle A^{1+2 \alpha-\gamma} U^{n}, P_{n} \sigma d W\right\rangle}{\left.\int_{0}^{T}\left|A^{1+\alpha} U^{n}\right|^{2} d t\right)} \\
& =\theta-\frac{\int_{0}^{T}\left\langle A^{1+2 \alpha-\gamma} U^{n}, \sum_{k} \phi_{k} d W^{k}\right\rangle}{\int_{0}^{T}\left|A^{1+\alpha} U^{n}\right|^{2} d t}
\end{aligned}
$$

Using Corollary 2.1, we obtain $\lim _{n \rightarrow \infty} \hat{\theta}_{n, T}=\theta$ in probability as $n \rightarrow \infty$.

b) Asymptotic Normality

Let

Then we have

$$
\sigma_{k}:=\lambda_{k}^{1+2 \alpha-\gamma} u_{k}, \quad \xi_{k}:=\int_{0}^{T} \sigma_{k}^{2} d t, \quad k \geq 1
$$

$$
\begin{gathered}
E\left(\xi_{k}\right) \sim \lambda_{k}^{2+4 \alpha-2 \gamma} \lambda_{k}^{-(1+2 \gamma)}=\lambda_{k}^{1+4 \alpha-4 \gamma}, \\
\operatorname{Var}\left(\xi_{k}\right) \sim \lambda_{k}^{4+8 \alpha-4 \gamma} \lambda_{k}^{-(3+4 \gamma)}=\lambda_{k}^{1+8 \alpha-8 \gamma} .
\end{gathered}
$$

Define $b_{n}:=\sum_{k=1}^{n} E\left(\xi_{k}\right)$. Under the given assumptions, $1+4 \alpha-4 \gamma<-1$, on $\alpha$, we have that $b_{n} \sim \lambda_{n}^{2+4 \alpha-4 \gamma}$, we infer that $b_{n}$ is increasing and unbounded. Moreover,

$$
\sum_{k=1}^{\infty} \frac{\operatorname{Var}\left(\xi_{k}\right)}{b_{k}^{2}} \leq c \sum_{k=1}^{\infty} k^{-3}<\infty .
$$

and therefore by LLN we conclude that

\section{Consequently}

$$
\lim _{n \rightarrow \infty} \frac{\sum_{k=1}^{n} \xi_{k}}{\sum_{k=1}^{n} E\left(\xi_{k}\right)}=1 \text { a.s. }
$$

$$
\frac{\int_{0}^{T}\left\langle A^{1+2 \alpha-\gamma} \bar{U}^{n}, \sum_{k} \phi_{k} d W^{k}\right\rangle}{\left(\int_{0}^{T}\left|A^{1+\alpha} \bar{U}^{n}\right|^{2} d t\right)^{1 / 2}} \rightarrow \mathcal{N}(0,1) .
$$

Noting that $1+\alpha>\gamma$ and $1+2 \alpha-\gamma>\gamma$,

$$
\begin{gathered}
\frac{\left(E \int_{0}^{T}\left|A^{1+2 \alpha-\gamma} \bar{U}^{n}\right|^{2} d t\right)^{1 / 2}}{\left(E \int_{0}^{T}\left|A^{1+\alpha} \bar{U}^{n}\right|^{2} d t\right)} \sim \sqrt{\frac{2 \theta}{\lambda_{1} T}} \frac{\alpha-\gamma+1}{\sqrt{\alpha-\gamma+1 / 2}} \frac{1}{n} . \\
n \frac{\int_{0}^{T}\left\langle A^{1+2 \alpha-\gamma} \bar{U}^{n}, \Sigma_{k} \phi_{k} d W^{k}\right\rangle}{\left(\int_{0}^{T}\left|A^{1+\alpha} \bar{U}^{n}\right|^{2} d t\right)} \rightarrow \mathcal{N}\left(0, \frac{2 \theta(\alpha-\gamma+1)^{2}}{\lambda_{1} T(\alpha-\gamma+1 / 2)}\right) .
\end{gathered}
$$


Thus

$$
n\left(\hat{\theta}_{n, T}-\theta\right) \rightarrow \mathcal{N}\left(0, \frac{2 \theta(\alpha-\gamma+1)^{2}}{\lambda_{1} T(\alpha-\gamma+1 / 2)}\right)
$$

Thus the asymptotic normality of $\hat{\theta}_{n}$ follows.

With $\delta_{2}=1$, in Proposition 2.1, we obtain

$$
n \frac{\int_{0}^{T}\left\langle A^{1+2 \alpha-\gamma} R_{n}, \sum_{k} \phi_{k} d W^{k}\right\rangle}{\int_{0}^{T}\left|A^{1+\alpha} U^{n}\right|^{2} d t} \rightarrow^{P} 0 .
$$

Thus

$$
\begin{gathered}
\frac{\frac{1}{n} \int_{0}^{T}\left\langle A^{1+2 \alpha-\gamma} R_{n}, \sum_{k} \phi_{k} d W^{k}\right\rangle}{\frac{1}{n^{2}} \int_{0}^{T}\left|A^{1+\alpha} U^{n}\right|^{2} d t} \rightarrow^{P} 0 . \\
\frac{\int_{0}^{T}\left|A^{1+\alpha} U^{n}\right|^{2} d t}{E\left(\int_{0}^{T}\left|A^{1+\alpha} U^{n}\right|^{2} d t\right)} \rightarrow^{\text {a.s. }} 1
\end{gathered}
$$

Remark 2.2 The smallest asymptotic variance corresponds to $\alpha=\gamma$.

Remark 2.3 When $\gamma \rightarrow \infty$, the system becomes smoother and when $\gamma=\infty$, the system becomes deterministic.

Remark 2.4

$$
E \int_{0}^{T}\left|A^{1+\alpha} \bar{U}^{n}\right|^{2} d t \sim n^{2 \alpha-2 \gamma+2}
$$

When $\alpha=\gamma$, this order is $O\left(n^{2}\right)$. Thus

$$
\frac{1}{n^{2}} E \int_{0}^{T}\left|A^{1+\alpha} \bar{U}^{n}\right|^{2} d t \sim C
$$

Hence

$$
\frac{1}{n} \int_{0}^{T}\left\langle A^{1+2 \alpha-\gamma} \bar{U}^{n}, \sum_{k} \phi_{k} d W^{k}\right\rangle \rightarrow^{D} \mathcal{N}(0, C)
$$

Note the unusual rate in comparison to the classical iid case where the rate is $\sqrt{n}$.

Remark 2.5 On the domain $\alpha>\gamma-1 / 2$, as a function of $\alpha$, the asymptotic variance $\frac{2 \theta(\alpha-\gamma+1)^{2}}{\lambda_{1} T(\alpha-\gamma+1 / 2)}$ reaches its minimum when $\alpha=\gamma$. In this case the asymptotic variance is given by $\frac{2 \theta}{T} \frac{2}{\lambda_{1}}$. Hence in this case

$$
n \sqrt{\frac{T}{2 \theta}} \sqrt{\frac{\lambda_{1}}{2}}\left(\tilde{\theta}_{n, T}-\theta\right) \rightarrow^{\mathcal{D}} \mathcal{N}(0,1) .
$$

\section{Alternative Proof by the Malliavin-Stein's Approach}


We give alternative proof from the tools of Malliavin calculus. We have

$$
\begin{gathered}
F_{n, T}:=\tilde{\theta}_{n, T}-\theta=\frac{\int_{0}^{T}\left\langle A^{1+2 \alpha-\gamma} \bar{U}^{n}, \sum_{k} \phi_{k} d W^{k}\right\rangle}{\left(\int_{0}^{T}\left|A^{1+\alpha} \bar{U}^{n}\right|^{2} d t\right)}=:-\frac{F_{1}(n, T)}{F_{2}(n, T)} \\
\hat{F}_{n, T}=\frac{\int_{0}^{T}\left\langle A^{1+2 \alpha-\gamma} \bar{U}^{n}, \sum_{k} \phi_{k} d W^{k}\right\rangle}{E\left(\int_{0}^{T}\left|A^{1+\alpha} \bar{U}^{n}\right|^{2} d t\right)}=:-\frac{F_{1}(n, T)}{C_{n, T}^{2}}
\end{gathered}
$$

where $C_{n, T}^{2}=E\left(F_{2}(n, T)\right)$. We note that $\hat{F}_{n, T}$ can be written as a double stochastic integral and it belongs to the second-order Wiener chaos.

Lemma 2.4 We have

$$
\sqrt{\operatorname{Var}\left(\frac{1}{2}\left\|C_{n, T} D \hat{F}_{n, T}\right\|_{H}^{2}\right)} \rightarrow 0 \text { as } n, T \rightarrow \infty
$$

where $D$ is the Malliavin derivative.

We show that $\left.C_{n, T} F_{n, T} \rightarrow^{\mathcal{D}} N\left(0, \sigma^{2}\right)\right)$ as $n, T \rightarrow \infty$ where $\sigma^{2}$ comes from the fact that $E\left(C_{n, T}^{2} \hat{F}_{n, T}^{2}\right)=\sigma^{2}$. We split $C_{n, T} F_{n, T}$ into

$$
\left.C_{n, T} F_{n, T}=C_{n, T}\left(F_{n, T}-\hat{F}_{n, T}\right)+C_{n, T} \hat{F}_{n, T}\right) .
$$

Note that

$$
\begin{gathered}
C_{n, T}\left(F_{n, T}-\hat{F}_{n, T}\right)=\frac{C_{n, T}^{2}}{F_{2}(n, T)} \frac{F_{1}(n, T)}{C_{n, T}}\left(1-\frac{F_{2}(n, T)}{C_{n, T}^{2}}\right) . \\
\frac{C_{n, T}^{2}}{F_{2}(n, T)} \rightarrow 1, \quad 1-\frac{F_{2}(n, T)}{C_{n, T}^{2}} \rightarrow^{\text {a.s. }} 0 \text { as } n, T \rightarrow \infty \\
\frac{F_{1}(n, T)}{C_{n, T}}=C_{n, T} \hat{F}_{n, T} \rightarrow^{\mathcal{D}} \mathcal{N}\left(0, \sigma^{2}\right) \text { as } n, T \rightarrow \infty .
\end{gathered}
$$

Hence by Slutzky's theorem,

$$
C_{n, T}\left(F_{n, T}-\hat{F}_{n, T}\right) \rightarrow^{\mathcal{D}} 0 \text { as } n, T \rightarrow \infty .
$$

which implies

$$
\begin{gathered}
C_{n, T}\left(F_{n, T}-\hat{F}_{n, T}\right) \rightarrow^{P} 0 \text { as } n, T \rightarrow \infty . \\
d_{T V}\left(C_{n, T} \hat{F}_{n, T}, \mathcal{N}\left(0, \sigma^{2}\right)\right) \rightarrow 0 \text { as } n, T \rightarrow \infty . \\
C_{n, T} \hat{F}_{n, T} \rightarrow{ }^{w} \mathcal{N}\left(0, \sigma^{2}\right) \text { as } n, T \rightarrow \infty . \\
\left.C_{n, T} F_{n, T} \rightarrow{ }^{w} \mathcal{N}\left(0, \sigma^{2}\right)\right) \text { as } n, T \rightarrow \infty .
\end{gathered}
$$




$$
\begin{gathered}
\frac{C_{n, T}}{\sigma} \approx \frac{\sqrt{2 \varpi} \sqrt{T} n}{8 \theta_{0}} \text { as } n, T \rightarrow \infty . \\
\sqrt{T} n F_{n, T} \rightarrow^{w} \mathcal{N}\left(0, \frac{\left.\sigma^{2}\left(8 \theta_{0}\right)^{2}\right)}{2 \varpi}\right) \text { as } n, T \rightarrow \infty .
\end{gathered}
$$

Hence

$$
\sqrt{T} n\left(\tilde{\theta}_{n, T}-\theta\right) \rightarrow^{\mathcal{D}} \mathcal{N}\left(0, \frac{\left.\sigma^{2}\left(8 \theta_{0}\right)^{2}\right)}{2 \varpi}\right) \text { as } n, T \rightarrow \infty
$$

\subsection{Berry-Esseen Bounds}

We obtain the rate of convergence to normality, i.e., the bound on the Kolmogorov distance between the distribution of the estimator and the normal distribution.

We consider the case $\alpha=\gamma$. In this case, the asymptotic variance is given by $\frac{2 \theta}{T} \frac{2}{\lambda_{1}}$.

Observe that

$$
n \sqrt{\frac{\lambda_{1}}{2}}\left(\frac{T}{2 \theta}\right)^{1 / 2}\left(\tilde{\theta}_{n, T}-\theta\right)=\sqrt{\frac{\lambda_{1}}{2} \frac{\frac{1}{n}\left(\frac{2 \theta}{T}\right)^{1 / 2} J_{n, T}}{\frac{1}{n^{2}}\left(\frac{2 \theta}{T}\right) I_{n, T}}}
$$

where

$$
J_{n, T}:=\theta I_{n, T}-\frac{T}{2} \quad \text { and } \quad I_{n, T}:=\sum_{k=1}^{n} \lambda_{k}^{2+2 \alpha} \int_{0}^{T} u_{k}(t)^{2} d t .
$$

Let $\Phi(\cdot)$ denote the standard normal distribution function. Throughout the paper, $C$ denotes a generic constant (which does not depend on $T$ and $x$ ). We have also tried to estimate the constant in the bound on normal approximation.

We start with some preliminary lemmas.

Lemma 2.2.1 For every $\delta>0$,

$$
P\left\{\left|\left(\frac{1}{n^{2}} \frac{2 \theta}{T} \frac{2}{\lambda_{1}}\right) I_{n, T}-1\right| \geq \delta\right\} \leq C n^{-2} \delta^{-2}
$$

Lemma 2.2.2

$$
\sup _{x \in \mathbb{R}}\left|P\left\{\sqrt{\frac{\lambda_{1}}{2}} \frac{1}{n}\left(\frac{2 \theta}{T}\right)^{1 / 2} J_{n, T} \leq x\right\}-\Phi(x)\right| \leq C T^{-1 / 2} n^{-1} .
$$

The constant $C$ in Lemma 2.2 is the constant in the classical Berry-Esseen theorem which is equal to 0.6751 . The following lemma gives the error rate on the difference of the characteristic function of the denominator of the MCE and the normal characteristic function. 
Lemma 2.2.3 (a) Let $\phi_{n, T}\left(z_{1}\right):=E \exp \left(z_{1} I_{n, T}\right), z_{1} \in \mathbb{C}$. Then $\phi_{n, T}\left(z_{1}\right)$ exists for $\left|z_{1}\right| \leq \delta$, for some $\delta>0$ and is given by

$$
\phi_{T}\left(z_{1}\right)=\exp \left(\frac{\theta T}{2}\right)\left[\frac{2 \gamma}{(\gamma-\theta) e^{-\gamma T}+(\gamma+\theta) e^{\gamma T}}\right]^{1 / 2}
$$

where $\gamma=\left(\theta^{2}-2 z_{1}\right)^{1 / 2}$ and we choose the principal branch of the square root.

(b) Let

$$
H_{n, T, x}:=\left(\frac{1}{n^{2}} \frac{2 \theta}{T} \frac{2}{\lambda_{1}}\right)^{1 / 2} J_{n, T}-\left(\frac{1}{n^{2}} \frac{2 \theta}{T} \frac{2}{\lambda_{1}} I_{n, T}-1\right) x
$$

Then for $|x| \leq 2(\log T)^{1 / 2}$ and for $|u| \leq \epsilon T^{1 / 2}$, where $\epsilon$ is sufficiently small

$$
\left|E \exp \left(i u H_{n, T, x}\right)-\exp \left(\frac{-u^{2}}{2}\right)\right| \leq C \exp \left(\frac{-u^{2}}{4}\right)\left(|u|+|u|^{3}\right) T^{-1 / 2} n^{-1} .
$$

Proof: To prove (b), observe that

$$
\begin{aligned}
& E \exp \left(i u H_{n, T, x}\right) \\
= & E \exp \left[-i u\left(\frac{2 \theta}{n^{2} T} \frac{2}{\lambda_{1}}\right)^{1 / 2} J_{n, T}-i u\left(\frac{2 \theta}{n^{2} T} \frac{2}{\lambda_{1}} I_{n, T}-1\right) x\right] \\
= & E \exp \left[-i u\left(\frac{2 \theta}{n^{2} T} \frac{2}{\lambda_{1}}\right)^{1 / 2}\left\{\theta I_{n, T}-\frac{T}{2}\right\}-i u\left(\frac{2 \theta}{n^{2} T} \frac{2}{\lambda_{1}} I_{n, T}-1\right) x\right] \\
= & E \exp \left(z_{1} I_{n, T}+z_{3}\right) \\
= & \exp \left(z_{3}\right) \phi_{T}\left(z_{1}\right)
\end{aligned}
$$

where $z_{1}:=-i u \theta \delta_{T, x}$ and $z_{3}:=\frac{i u T}{2} \delta_{T, x}$ with $\delta_{T, x}:=\left(\frac{2 \theta}{n^{2} T} \frac{2}{\lambda_{1}}\right)^{1 / 2}+\frac{2 x}{T}$.

Note that $\phi_{T}\left(z_{1}\right)$ satisfies the conditions of (a) by choosing $\epsilon$ sufficiently small. Let $\alpha_{1, T}(u)$, $\alpha_{2, T}(u), \alpha_{3, T}(u)$ and $\alpha_{4, T}(u)$ be functions which are $O\left(|u| T^{-1 / 2}\right), O\left(|u|^{2} T^{-1 / 2}\right), O\left(|u|^{3} T^{-3 / 2}\right)$ and $O\left(|u|^{3} T^{-1 / 2}\right)$ respectively. Note that for the given range of values of $x$ and $u$, the conditions on $z_{1}$ for part (a) of Lemma are satisfied. Further, with $\beta_{T}(u):=1+i u \frac{\delta_{T, x}}{\theta}+\frac{u^{2} \delta_{T, x}^{2}}{2 \theta^{2}}$,

$$
\begin{aligned}
\gamma & =\left(\theta^{2}-2 z_{1}\right)^{1 / 2} \\
& =\theta\left[1-\frac{z_{1}}{\theta^{2}}-\frac{z_{1}^{2}}{2 \theta^{4}}+\frac{z_{1}^{3}}{2 \theta^{8}}+\cdots\right] \\
& =\theta\left[1+i u \frac{\delta_{T, x}}{\theta}+\frac{u^{2} \delta_{T, x}^{2}}{2 \theta^{2}}+\frac{i u^{3} \delta_{T, x}^{3}}{2 \theta^{3}}+\cdots\right] \\
& =\theta\left[1+\alpha_{1, T}(u)+\alpha_{2, T}(u)+\alpha_{3, T}(u)\right] \\
& =\theta \beta_{T}(u)+\alpha_{3, T}(u) \\
& =\theta\left[1+\alpha_{1, T}(u)\right] .
\end{aligned}
$$


Thus

$$
\gamma-\theta=\alpha_{1, T}, \gamma+\theta=2 \theta+\alpha_{1, T}
$$

Hence the above expectation equals

$$
\begin{aligned}
& \exp \left(z_{3}+\frac{\theta T}{2}\right)\left[\frac{2 \theta \beta_{T}(u)+\alpha_{3, T}(u)}{\alpha_{1, T} \exp \left\{-\theta T \beta_{T}(u)+\alpha_{4, T}(u)\right\}+\left(2 \theta+\alpha_{1, T}(u)\right) \exp \left\{\theta T \beta_{T}(u)+\alpha_{4, T}(u)\right\}}\right]^{1 / 2} \\
= & {\left[\frac{1+\alpha_{1, T}(u)}{\alpha_{1, T} \exp \left(\chi_{T}(u)\right)+\left(1+\alpha_{1, T}(u)\right) \exp \left(\psi_{T}(t)\right)}\right]^{1 / 2} }
\end{aligned}
$$

where

$$
\begin{aligned}
\chi_{T}(u) & =-\theta T \beta_{T}(u)+\alpha_{4, T}(u)-2 z_{3}-\theta T \\
& =-2 \theta T+\alpha_{1, T}(u)+t^{2} \alpha_{1, T}(u)
\end{aligned}
$$

and

$$
\begin{aligned}
\psi_{T}(u) & =\theta T \beta_{T}(u)+\alpha_{4, T}(u)-2 z_{3}-\theta T \\
& =\theta T\left[1+i u \frac{\delta_{T, x}}{\theta}+\frac{u^{2} \delta_{T, x}^{2}}{2 \theta^{2}}\right]+\alpha_{4, T}(u)-i u T \delta_{T, x}-\theta T \\
& =\frac{u^{2} T}{2 \theta}\left[\left(\frac{2 \theta}{T}\right)^{1 / 2}+\frac{2 x}{T}\right]^{2} \\
& =u^{2}+u^{2} \alpha_{1, T}(u) .
\end{aligned}
$$

Hence, for the given range of values of $u, \chi_{T}(t)-\psi_{T}(u) \leq-\theta T$.

Hence the above expectation equals

$$
\begin{aligned}
& \exp \left(-\frac{u^{2}}{2}\right)\left(1+\alpha_{1, T}\right)^{1 / 2}\left[\alpha_{1, T} \exp \left\{-2 \theta T+\alpha_{1, T}+t^{2} \alpha_{1, T}\right\}+\left(1+\alpha_{1, T}(t)\right) \exp \left\{t^{2} \alpha_{1, T}(t)\right\}\right]^{-1 / 2} \\
= & \exp \left(-\frac{u^{2}}{2}\right)\left[1+\alpha_{1, T}\right)\left(1+\alpha_{1, T}\left(1+\alpha_{1, T}\right) \exp \left\{-\theta T+\alpha_{1, T}+t^{2} \alpha_{1, T}\right\}\right] \exp \left(u^{2} \alpha_{1, T}(u)\right) .
\end{aligned}
$$

This completes the proof of the lemma.

To obtain the rate of normal approximation for the MCE, we need the following estimate on the tail behavior of the MCE.

Lemma 2.2.4

$$
P\left\{\left(\frac{n^{2} T}{2 \theta} \frac{\lambda_{1}}{2}\right)^{1 / 2}\left|\tilde{\theta}_{n, T}-\theta\right| \geq 2(\log T)^{1 / 2}\right\} \leq C T^{-1 / 2} n^{-1}
$$


Proof : Observe that

$$
\begin{aligned}
& P\left\{\left(\frac{n^{2} T}{2 \theta} \frac{\lambda_{1}}{2}\right)^{1 / 2}\left|\tilde{\theta}_{n, T}-\theta\right| \geq 2\left(\log n^{2} T\right)^{1 / 2}\right\} \\
= & P\left\{\left|\frac{\left(\frac{2 \theta}{n^{2} T} \frac{2}{\lambda_{1}}\right)^{1 / 2} J_{n, T}}{\left(\frac{2 \theta}{n^{2} T} \frac{2}{\lambda_{1}}\right) I_{n, T}}\right| \geq 2\left(\log n^{2} T\right)^{1 / 2}\right\} \\
\leq & P\left\{\left|\left(\frac{2 \theta}{n^{2} T} \frac{2}{\lambda_{1}}\right)^{1 / 2} J_{n, T}\right| \geq(\log T)^{1 / 2}\right\}+P\left\{\left|\frac{2 \theta}{n^{2} T} \frac{2}{\lambda_{1}} I_{n, T}\right| \leq \frac{1}{2}\right\} \\
\leq & \left|P\left\{\left(\frac{2 \theta}{n^{2} T} \frac{2}{\lambda_{1}}\right)^{1 / 2}\left|J_{n, T}\right| \geq\left(\log n^{2} T\right)^{1 / 2}\right\}-2 \Phi\left(-\left(\log n^{2} T\right)^{1 / 2}\right)\right| \\
& +2 \Phi\left(-(\log T)^{1 / 2}\right)+P\left\{\left|\frac{2 \theta}{n^{2} T} \frac{2}{\lambda_{1}} I_{n, T}-1\right| \geq \frac{1}{2}\right\} \\
\leq & \sup _{x \in \mathbb{R}}\left|P\left\{\left(\frac{2 \theta}{n^{2} T} \frac{2}{\lambda_{1}}\right)^{1 / 2}\left|J_{n, T}\right| \geq x\right\}-2 \Phi(-x)\right| \\
\leq & \sup _{x \in \mathbb{R}}\left|P\left\{\left(\frac{2 \theta}{n^{2} T} \frac{2}{\lambda_{1}}\right)^{1 / 2}\left|J_{n, T}\right| \geq x\right\}-2 \Phi(-x)\right| \\
& +2 \Phi\left(-\left(\log n^{2} T\right)^{1 / 2}\right)+P\left\{\left|\left(\frac{2 \theta}{n^{2} T}\right) \frac{2}{\lambda_{1}} I_{n, T}-1\right| \geq \frac{1}{2}\right\} \\
\leq & C T^{-1 / 2} n^{-1}+C\left(n^{2} T \log n^{2} T\right)^{-1 / 2}+C T^{-1} n^{-2} \\
\leq & C T^{-1 / 2} n^{-1} .
\end{aligned}
$$

The bounds for the first and the third terms come from Lemma 2.2 and Lemma 2.1 respectively and that for the middle term comes from Feller (1957, p. 166).

Now we are ready to obtain the uniform rate of normal approximation of the distribution of the MCE.

\section{Theorem 2.2.5}

$$
\sup _{x \in \mathbb{R}}\left|P\left\{\left(\frac{n^{2} T}{2 \theta} \frac{\lambda_{1}}{2}\right)^{1 / 2}\left(\tilde{\theta}_{n, T}-\theta\right) \leq x\right\}-\Phi(x)\right| \leq C_{\theta} T^{-1 / 2} n^{-1} .
$$

Proof: We shall consider two possibilities (i) and (ii).

(i) $|x|>2\left(\log n^{2} T\right)^{1 / 2}$.

We shall give a proof for the case $x>2\left(\log n^{2} T\right)^{1 / 2}$. The proof for the case $x<-2\left(\log n^{2} T\right)^{1 / 2}$ runs similarly. Note that

$$
\left|P\left\{\left(\frac{n^{2} T}{2 \theta} \frac{\lambda_{1}}{2}\right)^{1 / 2}\left(\tilde{\theta}_{n, T}-\theta\right) \leq x\right\}-\Phi(x)\right| \leq P\left\{\left(\frac{n^{2} T}{2 \theta} \frac{\lambda_{1}}{2}\right)^{1 / 2}\left(\tilde{\theta}_{n, T}-\theta\right) \geq x\right\}+\Phi(-x) .
$$

But $\Phi(-x) \leq \Phi\left(-2\left(\log n^{2} T\right)^{1 / 2}\right) \leq C T^{-2} n^{-4}$. See Feller (1957, p. 166).

Moreover by Lemma 2.4, we have

$$
P\left\{\left(\frac{n^{2} T}{2 \theta} \frac{\lambda_{1}}{2}\right)^{1 / 2}\left(\tilde{\theta}_{n, T}-\theta\right) \geq 2\left(\log n^{2} T\right)^{1 / 2}\right\} \leq C T^{-1 / 2} n^{-1} .
$$


Hence

$$
\left|P\left\{\left(\frac{n^{2} T}{2 \theta} \frac{\lambda_{1}}{2}\right)^{1 / 2}\left(\tilde{\theta}_{n, T}-\theta\right) \leq x\right\}-\Phi(x)\right| \leq C T^{-1 / 2} n^{-1}
$$

(ii) $|x| \leq 2\left(\log n^{2} T\right)^{1 / 2}$

$$
\text { Let } \quad A_{n, T}:=\left\{\left(\frac{n^{2} T}{2 \theta} \frac{\lambda_{1}}{2}\right)^{1 / 2}\left|\tilde{\theta}_{n, T}-\theta\right| \leq 2\left(\log n^{2} T\right)^{1 / 2}\right\} \text { and } B_{n, T}:=\left\{\frac{I_{n, T}}{n^{2} T}>c_{0}\right\}
$$

where $0<c_{0}<\frac{1}{2 \theta}$. By Lemma 2.4, we have

$$
P\left(A_{n, T}^{c}\right) \leq C T^{-1 / 2} n^{-1}
$$

By Lemma 2.1, we have

$$
\begin{gathered}
P\left(B_{n, T}^{c}\right)=P\left\{\frac{2 \theta}{n^{2} T} \frac{2}{\lambda_{1}} I_{n, T}-1<2 \theta c_{0}-1\right\} \\
<P\left\{\left|\frac{2 \theta}{n^{2} T} \frac{2}{\lambda_{1}} I_{n, T}-1\right|>1-2 \theta c_{0}\right\} \leq C T^{-1} n^{-2} .
\end{gathered}
$$

Let $b_{0}$ be some positive number. On the set $A_{n, T} \cap B_{n, T}$ for all $T>T_{0}$ and $n>n_{0}$ with $4 b_{0}\left(\log n_{0}^{2} T_{0}\right)^{1 / 2}\left(\frac{2 \theta}{n_{0}^{2} T_{0}} \frac{2}{\lambda_{1}}\right)^{1 / 2} \leq c_{0}$, we have

$$
\begin{aligned}
& \left(\frac{n^{2} T}{2 \theta} \frac{\lambda_{1}}{2}\right)^{1 / 2}\left(\tilde{\theta}_{n, T}-\theta\right) \leq x \\
\Rightarrow & I_{n, T}+b_{0} n^{2} T\left(\tilde{\theta}_{n, T}-\theta\right)<I_{n, T}+\left(\frac{n^{2} T}{2 \theta} \frac{\lambda_{1}}{2}\right)^{1 / 2} 2 b_{0} \theta x \\
\Rightarrow & \left(\frac{n^{2} T}{2 \theta} \frac{\lambda_{1}}{2}\right)^{1 / 2}\left(\tilde{\theta}_{n, T}-\theta\right)\left[I_{n, T}+b_{0} n^{2} T\left(\theta_{T}-\theta\right)\right]<x\left[I_{n, T}+\left(\frac{n^{2} T}{2 \theta} \frac{\lambda_{1}}{2}\right)^{1 / 2} 2 b_{0} \theta x\right] \\
\Rightarrow & \left(\tilde{\theta}_{n, T}-\theta\right) I_{n, T}+b_{0} n^{2} T\left(\theta_{n, T}-\theta\right)^{2}<\left(\frac{2 \theta}{n^{2} T} \frac{2}{\lambda_{1}}\right)^{1 / 2} I_{n, T} x+2 b_{0} \theta x^{2} \\
\Rightarrow & -J_{n, T}+\left(\tilde{\theta}_{n, T}-\theta\right) I_{n, T}+b_{0} n^{2} T\left(\tilde{\theta}_{n, T}-\theta\right)^{2}<-J_{n, T}+\left(\frac{2 \theta}{n^{2} T} \frac{2}{\lambda_{1}}\right)^{1 / 2} I_{n, T} x+2 b_{0} \theta x^{2} \\
\Rightarrow & 0<-J_{n, T}+\left(\frac{2 \theta}{n^{2} T} \frac{2}{\lambda_{1}}\right)^{1 / 2} I_{n, T} x+2 b_{0} \theta x^{2}
\end{aligned}
$$

since

$$
\begin{aligned}
& I_{n, T}+b_{0} n^{2} T\left(\tilde{\theta}_{n, T}-\theta\right) \\
> & n^{2} n^{2} T c_{0}+b_{0} n^{2} T\left(\tilde{\theta}_{n, T}-\theta\right) \\
> & 4 b_{0}\left(\log n^{2} T\right)^{1 / 2}\left(\frac{2 \theta}{n^{2} T} \frac{2}{\lambda_{1}}\right)^{1 / 2}-2 b_{0}\left(\log n^{2} T\right)^{1 / 2}\left(\frac{2 \theta}{n^{2} T}\right)^{1 / 2} \\
= & 2 b_{0}\left(\log n^{2} T\right)^{1 / 2}\left(\frac{2 \theta}{n^{2} T} \frac{2}{\lambda_{1}}\right)^{1 / 2}>0 .
\end{aligned}
$$


On the other hand, on the set $A_{n, T} \cap B_{n, T}$ for all $T>T_{0}$ and $n>n_{0}$ with $4 b_{0}\left(\log n_{0}^{2} T_{0}\right)^{1 / 2}\left(\frac{2 \theta}{n_{0}^{2} T_{0}}\right)^{1 / 2} \leq c_{0}$, we have

$$
\begin{aligned}
& \left(\frac{n^{2} T}{2 \theta} \frac{\lambda_{1}}{2}\right)^{1 / 2}\left(\tilde{\theta}_{n, T}-\theta\right)>x \\
\Rightarrow & I_{n, T}-b_{0} T\left(\tilde{\theta}_{n, T}-\theta\right)<I_{n, T}-\left(\frac{n^{2} T}{2 \theta} \frac{\lambda_{1}}{2}\right)^{1 / 2} 2 b_{0} \theta x \\
\Rightarrow & \left(\frac{n^{2} T}{2 \theta} \frac{\lambda_{1}}{2}\right)^{1 / 2}\left(\tilde{\theta}_{n, T}-\theta\right)\left[I_{n, T}-b_{0} T\left(\theta_{n, T}-\theta\right)\right]>x\left[I_{n, T}-\left(\frac{n^{2} T}{2 \theta} \frac{\lambda_{1}}{2}\right)^{1 / 2} 2 b_{0} \theta x\right] \\
\Rightarrow & \left(\hat{\theta}_{n, T}-\theta\right) I_{n, T}-b_{0} T\left(\tilde{\theta}_{T}-\theta\right)^{2}>\left(\frac{2 \theta}{n^{2} T}\right)^{1 / 2} I_{n, T} x-2 b_{0} \theta x^{2} \\
\Rightarrow & -J_{n, T}+\left(\tilde{\theta}_{n, T}-\theta\right) I_{T}-b_{0} T\left(\tilde{\theta}_{n, T}-\theta\right)^{2}>-J_{n, T}+\left(\frac{2 \theta}{n^{2} T}\right)^{1 / 2} I_{n, T} x-2 b_{0} \theta x^{2} \\
\Rightarrow & 0>-J_{n, T}+\left(\frac{2 \theta}{n^{2} T}\right)^{1 / 2} I_{n, T} x-2 b_{0} \theta x^{2}
\end{aligned}
$$

since

$$
\begin{aligned}
& I_{n, T}-b_{0} n^{2} T\left(\tilde{\theta}_{n, T}-\theta\right) \\
> & n^{2} T c_{0}-b_{0} n^{2} T\left(\tilde{\theta}_{n, T}-\theta\right) \\
> & 4 b_{0}\left(\log n^{2} T\right)^{1 / 2}\left(\frac{2 \theta}{n^{2} T}\right)^{1 / 2}-2 b_{0}\left(\log n^{2} T\right)^{1 / 2}\left(\frac{2 \theta}{n^{2} T}\right)^{1 / 2} \\
= & 2 b_{0}\left(\log n^{2} T\right)^{1 / 2}\left(\frac{2 \theta}{n^{2} T}\right)^{1 / 2}>0 .
\end{aligned}
$$

Hence

$$
0<-J_{n, T}+\left(\frac{2 \theta}{n^{2} T}\right)^{1 / 2} I_{n, T} x-2 b_{0} \theta x^{2} \Rightarrow\left(\frac{n^{2} T}{2 \theta} \frac{\lambda_{1}}{2}\right)^{1 / 2}\left(\tilde{\theta}_{n, T}-\theta\right) \leq x
$$

Letting

$$
M_{n, T, x}^{ \pm}:=\left\{-J_{n, T}+\left(\frac{2 \theta}{n^{2} T} \frac{2}{\lambda_{1}}\right)^{1 / 2} I_{n, T} x \pm 2 b_{0} \theta x^{2}>0\right\}
$$

we obtain

$$
M_{n, T, x}^{-} \cap A_{n, T} \cap B_{n, T} \subseteq A_{n, T} \cap B_{n, T} \cap\left\{\left(\frac{n^{2} T}{2 \theta} \frac{\lambda_{1}}{2}\right)^{1 / 2}\left(\tilde{\theta}_{n, T}-\theta\right) \leq x\right\} \subseteq M_{n, T, x}^{+} \cap A_{n, T} \cap B_{n, T}
$$

If it is shown that

$$
\left|P\left\{M_{n, T, x}^{ \pm}\right\}-\Phi(x)\right| \leq C T^{-1 / 2} n^{-1}
$$

for all $T>T_{0}$ and $n>n_{0}$ and $|x| \leq 2\left(\log n^{2} T\right)^{1 / 2}$, then the theorem would follow from (2.1) (2.3). 
We shall prove (2.4) for $M_{n, T, x}^{+}$. The proof for $M_{n, T, x}^{-}$is analogous.

Observe that

$$
\begin{aligned}
= & \left|P\left\{M_{n, T, x}^{+}\right\}-\Phi(x)\right| \\
= & P\left\{\left(\frac{2 \theta}{n^{2} T} \frac{2}{\lambda_{1}}\right)^{1 / 2} J_{n, T}-\left(\frac{2 \theta}{n^{2} T} \frac{2}{\lambda_{1}} I_{n, T}-1\right) x<x+2\left(\frac{2 \theta}{n^{2} T} \frac{2}{\lambda_{1}}\right)^{1 / 2} b_{0} \theta x^{2}\right\}-\Phi(x) \mid \\
\leq & \sup _{y \in \mathbb{R}}\left|P\left\{\left(\frac{2 \theta}{n^{2} T} \frac{2}{\lambda_{1}}\right)^{1 / 2} J_{n, T}-\left(\frac{2 \theta}{n^{2} T} \frac{2}{\lambda_{1}} I_{n, T}-1\right) x \leq y\right\}-\Phi(y)\right| \\
& +\left|\Phi\left(x+\left(\frac{2 \theta}{n^{2} T} \frac{2}{\lambda_{1}}\right)^{1 / 2} b_{0} \theta x^{2}\right)-\Phi(x)\right| \\
=: & D_{1}+D_{2} .
\end{aligned}
$$

Lemma 2.3 (b) and Esseen's lemma immediately yield

$$
D_{1} \leq C T^{-1 / 2} n^{-1}
$$

On the other hand, for all $T>T_{0}$ and $n>n_{0}$,

$$
D_{2} \leq 2\left(\frac{2 \theta}{n^{2} T} \frac{2}{\lambda_{1}}\right)^{1 / 2} b_{0} \theta x^{2}(2 \pi)^{-1 / 2} \exp \left(-\bar{x}^{2} / 2\right)
$$

where

$$
|\bar{x}-x| \leq 2\left(\frac{2 \theta}{n^{2} T} \frac{2}{\lambda_{1}}\right)^{1 / 2} b_{0} \theta x^{2} .
$$

Since $|x| \leq 2\left(\log n^{2} T\right)^{1 / 2}$, it follows that $|\bar{x}|>|x| / 2$ for all $T>T_{0}$ and $n>n_{0}$, and consequently

$$
\begin{aligned}
D_{2} & \leq 2\left(\frac{2 \theta}{n^{2} T}\right)^{1 / 2} b_{0} \theta x^{2}(2 \pi)^{-1 / 2} x^{2} \exp \left(-x^{2} / 8\right) \\
& \leq C T^{-1 / 2} n^{-1}
\end{aligned}
$$

From (2.5) - (2.7), we obtain

$$
\left|P\left\{M_{n, T, x}^{+}\right\}-\Phi(x)\right| \leq C T^{-1 / 2} n^{-1}
$$

This completes the proof of the theorem.

The previous theorem does not give the precise estimate of the constant in the inequality. The following theorem gives a precise estimate of the constant.

Theorem 2.2.6 We have for all $T \geq T_{1}$, there exists $\epsilon(\theta)>0$ such that

$$
\begin{aligned}
& \sup _{x \in \mathbb{R}}\left|P\left\{\left(\frac{n^{2} T}{2 \theta} \frac{\lambda_{1}}{2}\right)^{1 / 2}\left(\tilde{\theta}_{n, T}-\theta\right) \leq x\right\}-\Phi(x)\right| \\
& \left.\leq\left[0.6751+0.4\left(\log n^{2} T\right)^{-1 / 2}\right)+a(\theta) T^{-1 / 2} n^{-1}\right] T^{-1 / 2} n^{-1}
\end{aligned}
$$


where $T_{1} \in \mathbb{R}^{+}$such that for all $T \geq T_{1}, \quad T^{-1} \log T \leq(b(\theta))^{2}$ and

$$
\begin{aligned}
b(\theta) & :=\frac{a_{2} a_{3}^{-1}\left(a_{3}-1\right)}{2 \theta} d(\theta), \\
d(\theta) & :=\min \left(\epsilon(\theta), \frac{a_{2}}{2 \theta a_{1} a_{3}}\right), \\
a(\theta) & :=\left(1-a_{2}\right)^{-2} a_{3}^{2}\left(1-a_{3}\right)^{-2} \epsilon(\theta) 2 \theta-\left(1-a_{2}\right)^{-2},
\end{aligned}
$$

$a_{1}, a_{2}, a_{3}$, are constants (not depending on $\theta$ ) satisfying: $a_{1}>1,0<a_{2}<1, a_{3}>1$.

Proof: Denote

$$
\begin{aligned}
F_{n, T, \theta} & :=\left\{\left(\frac{2 \theta}{n^{2} T} \frac{2}{\lambda_{1}}\right) I_{n, T}>a_{2}\right\}, \\
G_{n, T, \theta, \epsilon} & :=\left\{\frac{J_{n, T}}{n^{2} T} \leq \epsilon\left(-\epsilon a_{1}+\frac{a_{2}}{2 \theta}\right)\right\}, \\
Q_{n, T, \theta} & :=\left\{\left(\frac{2 \theta}{n^{2} T} \frac{2}{\lambda_{1}}\right)^{1 / 2} J_{n, T}>\left(\log n^{2} T\right)^{1 / 2}\right\}, \\
R_{n, T, \theta} & :=F_{n, T, \theta} \cap G_{n, T, \theta, d(\theta)}, \\
r & :=a_{2}^{-1} a_{3}\left(a_{3}-1\right)^{-1}
\end{aligned}
$$

where $a_{1}>1, \quad 0<a_{2}<1$ and $a_{3}>1$.

We obtain for all $\epsilon \in(0, \epsilon(\theta)]$,

$$
F_{n, T, \theta} \cap G_{n, T, \theta, \epsilon} \subset\left\{\left|\tilde{\theta}_{n, T}-\theta\right|<\epsilon\right\} .
$$

We shall consider two possibilities (i) and (ii).

(i) $|x|>r(\log T)^{1 / 2}$.

We shall give a proof for the case $x<-r\left(\log n^{2} T\right)^{1 / 2}$. The proof for the case $x>r\left(\log n^{2} T\right)^{1 / 2}$ runs similarly.

Observe that

$$
\begin{aligned}
& \left\{\left(\frac{n^{2} T}{2 \theta} \frac{\lambda_{1}}{2}\right)^{1 / 2}\left(\tilde{\theta}_{n, T}-\theta\right) \leq x\right\} \\
\subset & \left\{\left(\frac{n^{2} T}{2 \theta} \frac{\lambda_{1}}{2}\right)^{1 / 2}\left(\tilde{\theta}_{n, T}-\theta\right) \leq-r\left(\log n^{2} T\right)^{1 / 2}\right\} \cap F_{n, T, \theta} \cap G_{n, T, \theta, d}(\theta) \\
\cup & F_{n, T, \theta}^{c} \cup G_{n, T, \theta, d(\theta)}^{c} \\
\subset & Q_{n, T, \theta} \cup F_{n, T, \theta}^{c} \cup G_{n, T, \theta, d}^{c}(\theta) .
\end{aligned}
$$

Hence

$$
P\left\{\left(\frac{n^{2} T}{2 \theta} \frac{\lambda_{1}}{2}\right)^{1 / 2}\left(\tilde{\theta}_{n, T}-\theta\right) \leq x\right\} \leq P\left(Q_{n, T, \theta}\right)+P\left(F_{n, T, \theta}^{c}\right)+P\left(G_{n, T, \theta, d(\theta)}^{c}\right) .
$$


By Lemma 2.2 above and Lemma 2 in Feller (1957, p.166), we have

$$
\begin{aligned}
P\left(Q_{n, T, \theta}\right) & =\left|P\left(Q_{n, T, \theta}\right)-\Phi\left(-\left(\log n^{2} T\right)^{1 / 2}\right)\right|+\Phi\left(-\left(\log n^{2} T\right)^{1 / 2}\right) \\
& \leq\left[0.6751+0.4\left(\log n^{2} T\right)^{-1 / 2}\right] T^{-1 / 2} n^{-1} .
\end{aligned}
$$

Further, for $r>1$,

$$
\begin{aligned}
\Phi(x) & \leq \Phi\left(-r\left(\log n^{2} T\right)^{1 / 2}\right) \\
& \leq \Phi\left(-\left(\log n^{2} T\right)^{1 / 2}\right) \\
& \leq(2 \pi)^{-1 / 2}\left(\log n^{2} T\right)^{-1 / 2} T^{-1 / 2} n^{-1} .
\end{aligned}
$$

Thus

$$
\begin{aligned}
& \left|P\left\{\left(\frac{n^{2} T}{2 \theta} \frac{\lambda_{1}}{2}\right)^{1 / 2}\left(\tilde{\theta}_{n, T}-\theta\right) \leq x\right\}-\Phi(x)\right| \\
\leq & {\left[0.6751+0.4\left(\log n^{2} T\right)^{-1 / 2}\right] T^{-1 / 2}+P\left(F_{n, T, \theta}^{c}\right)+P\left(G_{n, T, \theta, d(\theta)}^{c}\right) . }
\end{aligned}
$$

(ii) $|x| \leq r\left(\log n^{2} T\right)^{1 / 2}$.

For all $T \in \mathbb{R}^{+}$with $n^{-1} T^{-1 / 2}\left(\log n^{2} T\right)^{1 / 2}<r^{-1}(2 \theta)^{-1 / 2} d(\theta)$, we have,

$$
\begin{aligned}
& \left\{\left(\frac{n^{2} T}{2 \theta} \frac{\lambda_{1}}{2}\right)^{1 / 2}\left(\tilde{\theta}_{n, T}-\theta\right) \leq x\right\} \cap F_{n, T, \theta} \cap G_{n, T, \theta, d(\theta)} \\
= & \left\{J_{n, T}+\left(\frac{2 \theta}{T n^{2}} \frac{2}{\lambda_{1}}\right)^{1 / 2} I_{n, T} x>0\right\} \cap F_{n, T, \theta} \cap G_{T, \theta, d(\theta)}
\end{aligned}
$$

by arguments similar to that in the proof of Theorem 2.5. From Lemma 2.2, we have

$$
\left|P\left\{J_{n, T}>0\right\}-\Phi(0)\right| \leq 0.6751 T^{-1 / 2} n^{-1} .
$$

Hence

$$
\begin{aligned}
& \left|P\left\{\left(\frac{n^{2} T}{2 \theta} \frac{\lambda_{1}}{2}\right)^{1 / 2}\left(\tilde{\theta}_{n, T}-\theta\right) \leq x\right\}-\Phi(x)\right| \\
\leq & 0.6751 T^{-1 / 2} n^{-1}+P\left(F_{n, T, \theta}^{c} \cup G_{n T, \theta, d(\theta)}^{c}\right)+|\Phi(0)-\Phi(x)| .
\end{aligned}
$$

This completes the proof of the theorem.

\section{Parameter Estimation from Discrete Observations}

The $n$ Fourier modes $u_{1}, u_{2}, \ldots, u_{n}$ are observed at $m$ time points $t_{i}, i=1,2, \ldots, m$. We denote $u_{k}\left(t_{i}\right)$ by $u_{i, k}$. Thus $u_{i, k}, i=1,2, \ldots, m ; k=1,2, \ldots, n$ are the observations.

Recall that the linearized MLE based on continuous time observations in $[0, T]$ is given by

$$
\hat{\theta}_{n, T}=-\frac{\int_{0}^{T}\left\langle A^{1+2 \alpha} U^{n}, d U^{n}\right\rangle}{\int_{0}^{T}\left|A^{1+\alpha} U^{n}\right|^{2} d t}=\frac{\sum_{k=1}^{n} \lambda_{k}^{1+2 \alpha} \int_{0}^{T} u_{k} d u_{k}}{\sum_{k=1}^{n} \lambda_{k}^{2+2 \alpha} \int_{0}^{T} u_{k}^{2} d t}
$$




$$
=\frac{\sum_{k=1}^{n} \lambda_{k}^{1+2 \alpha}\left(u_{k}^{2}(T)-u_{k}^{2}(0)-T \lambda_{k}^{-2 \gamma}\right)}{2 \sum_{k=1}^{n} \lambda_{k}^{2+2 \alpha} \int_{0}^{T} u_{k}^{2} d t} .
$$

By using Itô (Euler) type approximation of the stochastic integral and rectangular approximation of the ordinary integral the approximate maximum likelihood estimator (AMLE) is given by

$$
\hat{\theta}_{m, n, T}=\frac{\sum_{k=1}^{n} \lambda_{k}^{1+2 \alpha} \sum_{i=1}^{m} u_{i-1, k}\left(u_{i, k}-u_{i-1, k}\right)}{2 \sum_{i=1}^{m} \sum_{k=1}^{n} \lambda_{k}^{2+2 \alpha} u_{i-1, k}^{2}\left(t_{i}-t_{i-1}\right)} .
$$

$\mathrm{By}$ applying Itô formula to the stochastic integral and rectangular approximation of the ordinary integral the approximate maximum likelihood estimator (AMLE1) is given by

$$
\hat{\theta}_{m, n, T, 1}=\frac{\sum_{k=1}^{n} \lambda_{k}^{1+2 \alpha}\left(u_{k}^{2}(T)-u_{k}^{2}(0)-T \sum_{k=1}^{n} \lambda_{k}^{1+2 \alpha-2 \gamma}\right.}{2 \sum_{i=1}^{m} \sum_{k=1}^{n} \lambda_{k}^{2+2 \alpha} u_{i-1, k}^{2}\left(t_{i}-t_{i-1}\right)} .
$$

By applying rectangular approximation of the ordinary integral the approximate minimum contrast estimator (AMCE) is given by

$$
\tilde{\theta}_{m, n, T}=\frac{-T \sum_{k=1}^{n} \lambda_{k}^{1+2 \alpha-2 \gamma}}{2 \sum_{i=1}^{m} \sum_{k=1}^{n} \lambda_{k}^{2+2 \alpha} u_{i-1, k}^{2}\left(t_{i}-t_{i-1}\right)} .
$$

By applying trapezoidal approximation of the ordinary integral the approximate minimum contrast estimator (AMCE1) is given by

$$
\tilde{\theta}_{m, n, T, 1}=\frac{-T \sum_{k=1}^{n} \lambda_{k}^{1+2 \alpha-2 \gamma}}{\sum_{i=1}^{m} \sum_{k=1}^{n} \lambda_{k}^{2+2 \alpha}\left[u_{i-1, k}^{2}+u_{i, k}^{2}\right]\left(t_{i}-t_{i-1}\right)} .
$$

We study Berry-Esseen bounds for these estimators in this section. Bishwal and Bose (2001) studied rates of convergence of approximate maximum likelihood estimators and Bishwal (2009) studied rates of convergence of approximate minimum contrast estimator in the one dimensional Ornstein-Uhlenbeck process.

\subsection{Berry-Esseen Bounds for AMLEs}

Let us introduce the notations

$$
Y_{m, n, T}=\sum_{i=1}^{m} \sum_{k=1}^{n} \lambda_{k}^{2+2 \alpha} u_{k}\left(t_{i-1}\right)\left[W_{k}\left(t_{i}\right)-W_{k}\left(t_{i-1}\right)\right], \quad Y_{n, T}=\sum_{k=1}^{n} \lambda_{k}^{2+2 \alpha} \int_{0}^{T} u_{k}(t) d W_{k}(t),
$$




$$
\begin{gathered}
Z_{m, n, T}=\sum_{i=1}^{m} \sum_{k=1}^{n} \lambda_{k}^{2+2 \alpha} u_{k}\left(t_{i-1}\right)\left[u_{k}\left(t_{i}\right)-u_{k}\left(t_{i-1}\right)\right], \quad Z_{n, T}=\sum_{k=1}^{n} \lambda_{k}^{2+2 \alpha} \int_{0}^{T} u_{k}(t) d u_{k}(t), \\
I_{m, n, T}=\sum_{i=1}^{m} \sum_{k=1}^{n} \lambda_{k}^{2+2 \alpha} u_{k}^{2}\left(t_{i-1}\right)\left(t_{i}-t_{i-1}\right), \quad I_{n, T}=\sum_{k=1}^{n} \lambda_{k}^{2+2 \alpha} \int_{0}^{T} u_{k}^{2}(t) d t, \\
V_{m, n, T}=\sum_{i=1}^{m} \sum_{k=1}^{n} \lambda_{k}^{2+2 \alpha} \int_{t_{i-1}}^{t_{i}} u_{k}\left(t_{i-1}\right)\left[u_{k}(t)-u_{k}\left(t_{i-1}\right)\right] d t, \quad \Upsilon=\left(\frac{\lambda_{1}^{\beta}}{(4 \beta / d+2) \theta_{0}}\right)^{1 / 2} .
\end{gathered}
$$

\section{Lemma 3.1.1}

(a) $E\left|Y_{m, n, T}-Y_{n, T}\right|^{2}=O\left(T^{2} n^{3} / m\right)$,

(b) $E\left|Z_{m, n, T}-Z_{n, T}\right|^{2}=O\left(T^{2} n^{3} / m\right)$,

(c) $E\left|I_{m, n, T}-I_{n, T}\right|^{2}=O\left(T^{4} n^{5} / m^{2}\right)$,

(d) $E\left|V_{m, n, T}\right|^{2}=O\left(T^{4} n^{5} / m^{2}\right)$.

Proof. Let $g_{k, i}(t):=u_{k}\left(t_{i-1}\right)-u_{k}(t)$ for $t_{i-1} \leq t<t_{i}, \quad i=1,2, \ldots, m, \quad k=1,2, \ldots, n$. Since

$$
\begin{gathered}
E\left|u_{k}\left(t_{i-1}\right)-u_{k}(t)\right|^{2 k} \leq C\left(\lambda_{k}^{-2 \gamma}\right)^{k}\left(t_{i-1}-t\right)^{k}, k=1,2, \ldots \\
E\left|u_{k}\left(t_{i-1}\right)+u_{k}(t)\right|^{2 k} \leq C\left(\lambda_{k}^{-2 \gamma-2 \beta} T\right)^{k}, k=1,2, \ldots
\end{gathered}
$$

(by (3.11) of Chapter 4), hence

$$
\begin{aligned}
& E\left|Y_{m, n, T}-Y_{n, T}\right|^{2} \\
= & E\left|\sum_{k=1}^{n} \lambda_{k}^{2+\alpha} \sum_{i=1}^{m} u_{k}\left(t_{i-1}\right)\left[W_{k}\left(t_{i}\right)-W_{k}\left(t_{i-1}\right)\right]-\int_{0}^{T} u_{k}(t) d W_{k}(t)\right|^{2} \\
= & \sum_{k=1}^{n} \lambda_{k}^{4+2 \alpha} E\left|\int_{0}^{T} g_{k, i}(t) d W_{k}(t)\right|^{2} \\
= & \sum_{k=1}^{n} \lambda_{k}^{4+2 \alpha} \int_{0}^{T} E\left(g_{k, i}^{2}(t)\right) d t \\
\leq & C \sum_{k=1}^{n} \lambda_{k}^{4+2 \alpha} \sum_{i=1}^{m} \int_{t_{i-1}}^{t_{i}}\left|t_{i-1}-t\right| d t \\
= & C \sum_{k=1}^{n} \lambda_{k}^{2+2 \alpha} n \frac{\left(t_{i}-t_{i-1}\right)^{2}}{2}=C \frac{T^{2} n^{3}}{m} .
\end{aligned}
$$

This completes the proof of (a).

Using (2.1) and the fact that

$$
u_{k}\left(t_{i}\right)-u_{k}\left(t_{i-1}\right)=\int_{t_{i-1}}^{t_{i}} \theta u_{k}(t) d t+W_{k}\left(t_{i}\right)-W_{k}\left(t_{i-1}\right)
$$


we obtain

$$
\begin{aligned}
& E\left|Z_{m, n, T}-Z_{T}\right|^{2} \\
= & E\left|\sum_{k=1}^{n} \lambda_{k}^{2+2 \alpha} \sum_{i=1}^{m} u_{k}\left(t_{i-1}\right)\left[X_{t_{i}}-u_{k}\left(t_{i-1}\right)\right]-\sum_{k=1}^{n} \lambda_{k}^{2+2 \alpha} \int_{0}^{T} u_{k}(t) d W_{k}(t)\right|^{2} \\
= & \sum_{k=1}^{n} \lambda_{k}^{2+2 \alpha} E \mid \sum_{i=1}^{m} \int_{t_{i-1}}^{t_{i}} \theta u_{k}(t) u_{k}\left(t_{i-1}\right) d t+\sum_{i=1}^{m} u_{k}\left(t_{i-1}\right)\left[W_{k}\left(t_{i}\right)-W_{k}\left(t_{i-1}\right)\right] \\
& -\int_{0}^{T} \theta X_{t}^{2} d t-\left.\int_{0}^{T} u_{k}(t) d W_{k}(t)\right|^{2} \\
\leq & \sum_{k=1}^{n} \lambda_{k}^{2+2 \alpha} 2 E\left|\sum_{i=1}^{m} u_{k}\left(t_{i-1}\right)\left[W_{k}\left(t_{i}\right)-W_{k}\left(t_{i-1}\right)\right]-\int_{0}^{T} u_{k}(t) d W_{k}(t)\right|^{2} \\
& +2 \theta^{2} \sum_{k=1}^{n} \lambda_{k}^{2+2 \alpha} E\left|\sum_{i=1}^{m} \int_{t_{i-1}}^{t_{i}} u_{k}(t)\left[u_{k}\left(t_{i-1}\right)-u_{k}(t)\right] d t\right|^{2} . \\
=: & N_{1}+N_{2} .
\end{aligned}
$$

$N_{1}$ is $O\left(\frac{T^{2} n^{2}}{m}\right)$ by Lemma 3.3.2(a). To estimate $N_{2}$ let

$$
\psi_{k, i}(t):=u_{k}(t)\left[u_{k}\left(t_{i-1}\right)-u_{k}(t)\right]
$$

for $t_{i-1} \leq t<t_{i}, \quad i=1,2, \ldots, n, \quad k=1,2, \ldots, m$. Then

$$
\begin{aligned}
& \sum_{k=1}^{n} \lambda_{k}^{2+2 \alpha} E\left|\sum_{i=1}^{m} \int_{t_{i-1}}^{t_{i}} \psi_{k, i}(t) d t\right|^{2} \\
= & \sum_{k=1}^{n} \lambda_{k}^{2+2 \alpha} \sum_{i=1}^{n} E\left|\int_{t_{i-1}}^{t_{i}} \psi_{k, i}(t) d t\right|^{2}+2 \sum_{k=1}^{n} \lambda_{k}^{2+2 \alpha} \sum_{i, j=1, i<j}^{m} E\left[\int_{t_{i-1}}^{t_{i}} \psi_{k, i}(t) d t \int_{t_{i-1}}^{t_{i}} \psi_{j}(s) d s\right] \\
= & M_{1}+M_{2} .
\end{aligned}
$$

By the boundedness of $E\left(u_{k}^{4}(t)\right)$ and (2.1) we have

$$
\begin{aligned}
& E\left(\psi_{k, i}^{2}(t)\right) \\
= & E\left\{u_{k}(t)^{2}\left[u_{k}\left(t_{i-1}\right)-u_{k}(t)\right]^{2}\right\} \\
\leq & \left\{E\left(u_{k}^{4}(t)\right)\right\}^{1 / 2}\left\{E\left[u_{k}\left(t_{i-1}\right)-u_{k}(t)\right]^{4}\right\}^{1 / 2} \\
\leq & C\left(t_{i-1}-t\right) .
\end{aligned}
$$

Note that

$$
\begin{aligned}
M_{1} & =\sum_{k=1}^{n} \lambda_{k}^{2+2 \alpha} \sum_{i=1}^{m} E\left|\int_{t_{i-1}}^{t_{i}} \psi_{i}(t) d t\right|^{2} \\
& \leq \sum_{k=1}^{n} \lambda_{k}^{2+2 \alpha} \sum_{i=1}^{m}\left(t_{i}-t_{i-1}\right) \int_{t_{i-1}}^{t_{i}} E\left(\psi^{2}(t)\right) d t \\
& \leq C \frac{T}{n} \sum_{k=1}^{n} \lambda_{k}^{2+2 \alpha} \sum_{i=1}^{m} \int_{t_{i-1}}^{t_{i}}\left(t-t_{i-1}\right) d t \\
& \leq C \frac{T}{n} \sum_{k=1}^{n} \lambda_{k}^{2+2 \alpha} \sum_{i=1}^{n}\left(t_{i}-t_{i-1}\right)^{2}=C \frac{T^{3} n^{4}}{m^{2}}
\end{aligned}
$$


and

$$
\begin{aligned}
M_{2} & =\sum_{k=1}^{n} \lambda_{k}^{2+2 \alpha_{2}} \sum_{i, j=1, i<j}^{m} E \int_{t_{i-1}}^{t_{i}} \int_{t_{j-1}}^{t_{i}}\left[\psi_{k, i}(t) \psi_{k, j}(s)\right] d t d s \\
& =\sum_{k=1}^{n} \lambda_{k}^{2+2 \alpha_{2}} \sum_{i, j=1, i<j}^{m} \int_{t_{i-1}}^{t_{i}} \int_{t_{j-1}}^{t_{j}} E\left[\psi_{i}(t) \psi_{k, j}(s)\right] d t d s .
\end{aligned}
$$

By Lemma 3.3.1, we have

$$
\begin{aligned}
& E\left[\psi_{k, i}(t) \psi_{k, j}(s)\right] \\
= & E\left[u_{k}(t)\left(u_{k}\left(t_{i-1}\right)-u_{k}(t)\right) u_{k}(s)\left(u_{k}\left(t_{j-1}\right)-u_{k}(s)\right)\right] \\
= & E\left[u_{k}(t)\left(u_{k}\left(t_{i-1}\right)-u_{k}(t)\right)\right] E\left[u_{k}(s)\left(u_{k}\left(t_{j-1}\right)-u_{k}(s)\right)\right] \\
+ & E\left[u_{k}(t) u_{k}(s)\right] E\left[\left(u_{k}\left(t_{i-1}\right)-u_{k}(t)\right)\left(u_{k}\left(t_{j-1}\right)-u_{k}(s)\right)\right] \\
+ & E\left[u_{k}(t)\left(u_{k}\left(t_{j-1}\right)-u_{k}(s)\right)\right] E\left[u_{k}(s)\left(u_{k}\left(t_{i-1}\right)-u_{k}(t)\right)\right] \\
=: & A_{1}+A_{2}+A_{3} .
\end{aligned}
$$

Note that

$$
u_{k}(t)=\lambda_{k}^{2+2 \alpha} \int_{0}^{t} e^{\theta \lambda_{1}(t-u)} d W_{u}, t \geq 0
$$

Let $a:=e^{\theta \lambda_{1}}$. For $s \geq t$, we have

$$
\begin{aligned}
& E\left(u_{k}(t) u_{k}(s)\right) \\
= & E\left(\int_{0}^{t} e^{\theta \lambda_{1}(t-u)} d W_{k}(u)\right)\left(\int_{0}^{s} e^{\theta \lambda_{1}(s-u)} d W_{k}(u)\right) \\
= & \int_{0}^{t} e^{\theta \lambda_{1}(t+s-2 u)} d u \\
= & \frac{1}{2 \theta \lambda_{1}}\left[a^{s+t}-a^{s-t}\right]
\end{aligned}
$$

Observe that

$$
\begin{aligned}
& E\left(u_{k}(t)-u_{k}\left(t_{i-1}\right)\right)\left(u_{k}(s)-u_{k}\left(t_{j-1}\right)\right) \\
= & E\left(u_{k}(t) u_{k}(s)\right)-E\left(u_{k}(t) u_{k}\left(t_{j-1}\right)\right)-E\left(u_{k}\left(t_{i-1}\right) u_{k}(s)\right)+E\left(u_{k}\left(t_{i-1}\right) u_{k}\left(t_{j-1}\right)\right) \\
= & \frac{1}{2 \theta}\left(a^{s}-a^{t_{j-1}}\right)\left[\left(a^{t}-a^{t_{i-1}}\right)+\left(a^{-t_{i-1}}-a^{-t}\right)\right] \\
= & \frac{1}{2 \theta}\left(s-t_{j-1}\right) a^{t^{*}}\left[\left(t-t_{i-1} a^{t^{* *}}+\left(t-t_{i-1}\right) a^{-t^{* * *}}\right]\right. \\
& \left(\text { where } t_{j-1}<t^{*}<s, t_{i-1}<t^{* *}, t^{* * *}<t\right) \\
\leq & \left.\frac{1}{2 \theta}\left(s-t_{j-1}\right) a^{t}\left(t-t_{i-1}\right) a^{t_{i-1}}+\left(s-t_{j-1}\right) a^{t}\left(t-t_{i-1}\right) a^{-t}\right] \\
\leq & C\left(s-t_{j-1}\right)\left(t-t_{i-1}\right) .
\end{aligned}
$$

Thus $A_{2} \leq C\left(s-t_{j-1}\right)\left(t-t_{i-1}\right)$ since $\left|E\left(u_{k}(t) u_{k}(s)\right)\right|$ is bounded. 
Next

$$
\begin{aligned}
& \left|E\left[u_{k}(t)\left(u_{k}\left(t_{i-1}\right)-u_{k}(t)\right)\right]\right| \\
= & \frac{1}{2|\theta|}\left[a^{t+t_{i-1}}-a^{t-t_{i-1}}-a^{2 t}+1\right] \\
= & \frac{1}{2|\theta|} a^{t}\left[a^{t_{i-1}}-a^{-t_{i-1}}-a^{t}+a^{-t}\right] \\
\leq & \frac{1}{2|\theta|} a^{t}\left(t-t_{i-1}\right)\left[a^{t_{i-1}}+a^{-t}\right] \\
\leq & C\left(t-t_{i-1}\right)
\end{aligned}
$$

and

$$
\begin{aligned}
& \mid E\left[u_{k}(s)\left(u_{k}(s)-u_{k}\left(t_{j-1}\right)\right] \mid\right. \\
= & \frac{1}{2|\theta|}\left[a^{2 s}-1-a^{s+t_{j-1}}+a^{s+t_{j-1}}\right] \\
= & \frac{1}{2|\theta|} a^{s}\left[a^{s}-a^{-s}-a^{t_{j-1}}+a^{-t_{j-1}}\right] \\
\leq & \frac{1}{2|\theta|} a^{s}\left(s-t_{j-1}\right)\left[a^{t_{j-1}}+a^{-s}\right] \\
\leq & C\left(s-t_{j-1}\right) .
\end{aligned}
$$

Thus $A_{1} \leq C\left(s-t_{j-1}\right)\left(t-t_{i-1}\right)$.

Next

$$
\begin{aligned}
& \mid E\left[u_{k}(t)\left(u_{k}(s)-u_{k}\left(t_{j-1}\right)\right] \mid\right. \\
= & \frac{1}{2|\theta|}\left[a^{s+t}-a^{s-t}-a^{t+t_{j-1}}+a^{t_{j-1}-t}\right] \\
= & \frac{1}{2|\theta|} a^{t}\left(a^{s}-a^{t_{j-1}}\right) \\
\leq & \frac{1}{2|\theta|} a^{t}\left(1-a^{-2 t}\right)\left(s-t_{j-1}\right) a^{t} \\
\leq & \left(a^{2 t}-1\right)\left(s-t_{j-1}\right) \\
\leq & C\left(s-t_{j-1}\right)
\end{aligned}
$$

and

$$
\begin{aligned}
& \mid E\left[u_{k}(s)\left(u_{k}(t)-u_{k}\left(t_{i-1}\right)\right] \mid\right. \\
= & \frac{1}{2|\theta|}\left[a^{t+s}-a^{s-t}-a^{s+t_{i-1}}+a^{s-t_{i-1}}\right] \\
= & \frac{1}{2|\theta|} a^{s}\left[a^{t}-a^{-t}-a^{t_{i-1}}+a^{-t_{i-1}}\right] \\
\leq & \frac{1}{2|\theta|} a^{s}\left(t-t_{i-1}\right)\left[a^{t_{i-1}}+a^{-t}\right] \\
\leq & C\left(t-t_{i-1}\right) .
\end{aligned}
$$


Thus $A_{3} \leq C\left(s-t_{j-1}\right)\left(t-t_{i-1}\right)$.

Hence $E\left[f_{i}(t) f_{j}(s)\right] \leq C\left(s-t_{j-1}\right)\left(t-t_{i-1}\right)$.

Thus

$$
\begin{aligned}
M_{2} & =2 \sum_{k=1}^{n} \lambda_{k}^{2+2 \alpha} \sum_{i, j=1, i<j}^{m} \int_{t_{i-1}}^{t_{i}} \int_{t_{j-1}}^{t_{j}} E\left[f_{i}(t) f_{j}(s)\right] d t d s \\
& \leq C \sum_{i, j=1, i<j}^{m} \int_{t_{i-1}}^{t_{i}} \int_{t_{j-1}}^{t_{j}}\left(t-t_{i-1}\right)\left(s-t_{j-1}\right) d t d s \\
& =C \sum_{k=1}^{n} \lambda_{k}^{2+2 \alpha} \sum_{i, j=1, i<j}^{m}\left(t_{i-1}-t_{i}\right)^{2}\left(t_{j-1}-t_{i}\right)^{2} \\
& =C n^{2} \sum_{k=1}^{n} \lambda_{k}^{2+2 \alpha}\left(\frac{T}{n}\right)^{4}=C \sum_{k=1}^{n} \lambda_{k}^{6} \frac{T^{4}}{m^{2}} .
\end{aligned}
$$

Hence, $N_{2}$ is $O\left(\frac{T^{3}}{n^{2}}\right)$. Combining $N_{1}$ and $N_{2}$ completes the proof of (b). We next prove (c). Let $\chi_{k, i}(t):=u_{k}^{2}\left(t_{i-1}\right)-u_{k}(t)^{2}, t_{i-1} \leq t<t_{i}, i=1,2, \ldots, m, k=1,2, \ldots, m$. Then

$$
\begin{aligned}
& E\left|I_{m, n, T}-I_{n, T}\right|^{2} \\
= & E\left|\sum_{k=1}^{n} \lambda_{k}^{4+2 \alpha} \sum_{i=1}^{m} u_{k}^{2}\left(t_{i-1}\right)\left(t_{i}-t_{i-1}\right)-\int_{0}^{T} u_{k}^{2}(t) d t\right|^{2} \\
= & E\left|\sum_{k=1}^{n} \lambda_{k}^{4+2 \alpha} \sum_{i=1}^{m} \int_{t_{i-1}}^{t_{i}}\left[u_{k}^{2}\left(t_{i-1}\right)-u_{k}^{2}(t)\right] d t\right|^{2} \\
= & \sum_{k=1}^{n} \lambda_{k}^{8+4 \alpha} E\left|\sum_{i=1}^{m} \int_{t_{i-1}}^{t_{i}} \chi_{k, i}(t) d t\right|^{2} \\
= & \sum_{k=1}^{n} \lambda_{k}^{8+4 \alpha} \sum_{i=1}^{m} E\left|\int_{t_{i-1}}^{t_{i}} \chi_{k, i}(t) d t\right|^{2}+2 \sum_{k=1}^{n} \lambda_{k}^{8+4 \alpha} \sum_{i, j=1, i<j}^{m} E \int_{t_{i-1}}^{t_{i}} \int_{t_{j-1}}^{t_{j}} \chi_{k, i}(t) \chi_{j}(s) d t d s \\
= & B_{1}+B_{2} .
\end{aligned}
$$

$$
\begin{aligned}
E \chi_{k, i}^{2}(t) & =E\left[u_{k}^{2}\left(t_{i-1}\right)-u_{k}(t)^{2}\right]^{2} \\
& =E\left[u_{k}\left(t_{i-1}\right)-u_{k}(t)\right]^{2}\left[u_{k}\left(t_{i-1}\right)+u_{k}(t)\right]^{2} \\
& \leq\left\{E\left[u_{k}\left(t_{i-1}\right)-u_{k}(t)\right]^{4}\right\}^{1 / 2}\left\{\left\{E\left[u_{k}\left(t_{i-1}\right)+u_{k}(t)\right]^{4}\right\}^{1 / 2}\right. \\
& \leq C\left(t-t_{i-1}\right)
\end{aligned}
$$


(by (3.1) and the boundedness of the second term)

$$
\begin{aligned}
B_{1} & =\sum_{k=1}^{n} \lambda_{k}^{2+2 \alpha} \sum_{i=1}^{m} E\left|\int_{t_{i-1}}^{t_{i}} \chi_{k, i}(t) d t\right|^{2} \\
& \leq \sum_{k=1}^{n} \lambda_{k}^{2+2 \alpha} \sum_{i=1}^{m}\left(t_{i}-t_{i-1}\right) \int_{t_{i-1}}^{t_{i}} E\left(\chi_{k, i}^{2}(t)\right) d t \\
& \leq C \frac{T}{n} \sum_{k=1}^{n} \lambda_{k}^{2+2 \alpha} \sum_{i=1}^{m} \int_{t_{i-1}}^{t_{i}}\left(t-t_{i-1}\right) d t \\
& \leq C \sum_{k=1}^{n} \lambda_{k}^{8} \frac{T^{3} n^{4}}{m^{2}} .
\end{aligned}
$$

Note that

$$
\begin{aligned}
& E\left[\chi_{i}(t) \chi_{j}(s)\right] \\
= & E\left(u_{k}\left(t_{i-1}\right)^{2}-u_{k}(t)^{2}\right)\left(u_{k}\left(t_{j-1}\right)^{2}-u_{k}(s)^{2}\right) \\
= & E\left(u_{k}\left(t_{i-1}\right)-u_{k}(t)\right)\left(u_{k}\left(t_{i-1}\right)+u_{k}(t)\right)\left(u_{k}\left(t_{j-1}\right)-u_{k}(s)\right)\left(u_{k}\left(t_{j-1}\right)+u_{k}(s)\right)
\end{aligned}
$$

Now using Lemma 3.3.1 and proceeding similar to the estimation of $M_{2}$ it is easy to see that

$$
B_{2} \leq C \sum_{k=1}^{n} \lambda_{k}^{8} \frac{T^{4}}{n^{2}} \leq \frac{T^{4} n^{5}}{m^{2}} .
$$

Combining $B_{1}$ and $B_{2}$, (c) follows.

We need the following elementary lemmas to prove our main results.

Lemma 3.1.2 Let $X, Y$ and $Z$ be any three random variables on a probability space $(\Omega, \mathcal{F}, P)$ with $P(Z>0)=1$. Then, for any $\epsilon>0$, we have

$$
\begin{aligned}
& \text { (a) } \sup _{x \in \mathbb{R}}|P\{X+Y \leq x\}-\Phi(x)| \leq \sup _{x \in \mathbb{R}}|P\{X \leq x\}-\Phi(x)|+P(|Y|>\epsilon)+\epsilon, \\
& \text { (b) } \sup _{x \in \mathbb{R}}\left|P\left\{\frac{X}{Z} \leq x\right\}-\Phi(x)\right| \leq \sup _{x \in \mathbb{R}}|P\{X \leq x\}-\Phi(x)|+P\{|Z-1|>\epsilon\}+\epsilon .
\end{aligned}
$$

Lemma 1.1 (a) is from Michel and Pfanzagl (1971) and proof of (b) is elementary. Proof of the following lemma is also elementary.

Lemma 3.1.3 Let $Q_{n}, R_{n}, Q$ and $R$ be random variables on the same probability space $(\Omega, \mathcal{F}, P)$ with $P\left(R_{n}>0\right)=1$ and $P(R>0)=1$. Suppose $\left|Q_{n}-Q\right|=O_{P}\left(\delta_{1 n}\right)$ and $\left|R_{n}-R\right|=O_{P}\left(\delta_{2 n}\right)$ where $\delta_{1 n}, \delta_{2 n} \rightarrow 0$ as $n \rightarrow \infty$. Then,

$$
\left|\frac{Q_{n}}{R_{n}}-\frac{Q}{R}\right|=O_{P}\left(\max \left(\delta_{1 n}, \delta_{2 n}\right)\right) .
$$


Lemma 3.1.4 (Wick's lemma) Let $\left(\xi_{1}, \xi_{2}, \xi_{3}, \xi_{4}\right)$ be a Gaussian random vector with zero mean. Then,

$$
E\left(\xi_{1} \xi_{2} \xi_{3} \xi_{4}\right)=E\left(\xi_{1} \xi_{2}\right) E\left(\xi_{3} \xi_{4}\right)+E\left(\xi_{1} \xi_{3}\right) E\left(\xi_{2} \xi_{4}\right)+E\left(\xi_{1} \xi_{4}\right) E\left(\xi_{2} \xi_{3}\right)
$$

Let $\Phi(\cdot)$ denote the standard normal distribution function. Throughout the paper $C$ denotes a generic constant (perhaps depending on $\theta$, but not on anything else).

We need the following lemmas in the sequel whose proofs are similar to those in Bishwal (2000a). Lemma 3.1.5 For every $\delta>0$,

$$
P\left\{\left|\frac{2 \theta}{T} \frac{2}{\lambda_{1}} I_{T}-1\right| \geq \delta\right\} \leq C T^{-1} \delta^{-2}
$$

Lemma 3.1.6

$$
\sup _{x \in \mathbb{R}}\left|P\left\{\left(-\frac{2 \theta}{T} \frac{2}{\lambda_{1}}\right)^{1 / 2}\left(\theta I_{T}-\frac{T}{2}\right) \leq x\right\}-\Phi(x)\right| \leq C T^{-1 / 2}
$$

Lemma 3.1.7

$$
\begin{gathered}
\text { (a) } E\left|I_{n, T}-I_{T}\right|^{2}=O\left(\frac{T^{4}}{n^{2}}\right) . \\
\text { (b) } E\left|\frac{I_{n, T}+J_{n, T}}{2}-I_{T}\right|^{2}=O\left(\frac{T^{4}}{n^{2}}\right) .
\end{gathered}
$$

Part (a) is similar to that in Bishwal and Bose (2001). The proof of part (b) is analogous to part (a). We omit the details.

Theorem 3.1.1 Let $\alpha_{n, T}:=\max \left(T^{-1 / 2}(\log T)^{1 / 2}, \frac{T^{2}}{n}(\log T)^{-1}, \frac{T^{4}}{n^{2}}(\log T)^{-1}\right)$. We have,

$$
\begin{aligned}
& \text { (a) } \sup _{x \in \mathbb{R}}\left|P\left\{\left(\frac{T}{-2 \theta} \frac{\lambda_{1}}{2}\right)^{1 / 2}\left(\hat{\theta}_{m, n, T}-\theta\right) \leq x\right\}-\Phi(x)\right|=O\left(\alpha_{n, T}\right) . \\
& \text { (b) } \sup _{x \in \mathbb{R}}\left|P\left\{I_{n, T}^{1 / 2}\left(\hat{\theta}_{m, n, T}-\theta\right) \leq x\right\}-\Phi(x)\right|=O\left(\alpha_{n, T}\right) . \\
& \text { (c) } \sup _{x \in \mathbb{R}}\left|P\left\{\left(\frac{T}{\left|2 \hat{\theta}_{m, n, T}\right|} \frac{\lambda_{1}}{2}\right)^{1 / 2}\left(\hat{\theta}_{m, n, T}-\theta\right) \leq x\right\}-\Phi(x)\right|=O\left(\alpha_{n, T}\right) .
\end{aligned}
$$

Proof: (a) It is easy to see that

$$
\hat{\theta}_{m, n, T}-\theta=\frac{Y_{m, n, T}}{I_{m, n, T}}+\theta \frac{V_{m, n, T}}{I_{m, n, T}}
$$


Hence

$$
\begin{aligned}
& \sup _{x \in \mathbb{R}}\left|P\left\{\left(\frac{T}{-2 \theta} \frac{\lambda_{1}}{2}\right)^{1 / 2}\left(\hat{\theta}_{m, n, T}-\theta\right) \leq x\right\}-\Phi(x)\right| \\
= & \sup _{x \in \mathbb{R}}\left|P\left\{\left(\frac{T}{-2 \theta} \frac{\lambda_{1}}{2}\right)^{1 / 2} \frac{Y_{m, n, T}}{I_{m, n, T}}+\left(\frac{T}{-2 \theta} \frac{\lambda_{1}}{2}\right)^{1 / 2} \theta \frac{V_{m, n, T}}{I_{m, n, T}} \leq x\right\}-\Phi(x)\right| \\
\leq & \sup _{x \in \mathbb{R}}\left|P\left\{\left(\frac{T}{-2 \theta} \frac{\lambda_{1}}{2}\right)^{1 / 2} \frac{Y_{m, n, T}}{I_{m, n, T}} \leq x\right\}-\Phi(x)\right| \\
& +P\left\{\left|\left(\frac{T}{-2 \theta} \frac{\lambda_{1}}{2}\right)^{1 / 2} \frac{V_{m, n, T}}{I_{m, n, T}}\right|>\epsilon\right\}+\epsilon . \\
=: & K_{1}+K_{2}+\epsilon .
\end{aligned}
$$

Note that by Lemma 1.2.1 (b)

$$
\begin{aligned}
& K_{1}=\sup _{x \in \mathbb{R}}\left|P\left\{\left(\frac{T}{-2 \theta} \frac{\lambda_{1}}{2}\right)^{1 / 2} \frac{Y_{m, n, T}}{I_{m, n, T}} \leq x\right\}-\Phi(x)\right| \\
& \begin{array}{l}
=\sup _{x \in \mathbb{R}}\left|P\left\{\frac{\left(-\frac{2 \theta}{T} \frac{2}{\lambda_{1}}\right)^{1 / 2} Y_{m, n, T}}{\left(\frac{-2 \theta}{T} \frac{2}{\lambda_{1}}\right) I_{m, n, T}} \leq x\right\}-\Phi(x)\right| \\
\leq \sup _{x \in \mathbb{R}} P\left\{\left(\frac{-2 \theta}{T} \frac{2}{\lambda_{1}}\right)^{1 / 2} Y_{m, n, T} \leq x\right\}-\Phi(x) \mid
\end{array} \\
& +P\left\{\left(\frac{-2 \theta}{T} \frac{2}{\lambda_{1}}\right) I_{m, n, T}-1>\epsilon\right\}+\epsilon \\
& =: J_{1}+J_{2}+\epsilon \text {. } \\
& J_{1}=\sup _{x \in \mathbb{R}}\left|P\left\{\left(\frac{-2 \theta}{T} \frac{2}{\lambda_{1}}\right)^{1 / 2}\left(Y_{m, n, T}-Y_{n, T}+Y_{n, T}\right) \leq x\right\}-\Phi(x)\right| \\
& \leq \sup _{x \in \mathbb{R}} P\left\{\left(\frac{-2 \theta}{T} \frac{2}{\lambda_{1}}\right)^{1 / 2} Y_{n, T} \leq x\right\}-\Phi(x) \mid \\
& +P\left\{\left(\frac{-2 \theta}{T} \frac{2}{\lambda_{1}}\right)^{1 / 2}\left|Y_{m, n, T}-Y_{n, T}\right|>\epsilon\right\}+\epsilon \\
& \leq C T^{-1 / 2}+\left(\frac{-2 \theta}{T} \frac{2}{\lambda_{1}}\right) \frac{E\left|Y_{m, n, T}-Y_{n, T}\right|^{2}}{\epsilon^{2}}+\epsilon \\
& \leq C T^{-1 / 2}+C \frac{T / n}{\epsilon^{2}}+\epsilon \text {. (by Corollary 1.2.3(a) and Lemma 3.3.2(a).) } \\
& J_{2}=P\left\{\left|\left(\frac{-2 \theta}{T} \frac{2}{\lambda_{1}}\right)\left(I_{m, n, T}-I_{n, T}+I_{n, T}\right)-1\right|>\epsilon\right\} \\
& \leq P\left\{\left|\left(\frac{-2 \theta}{T} \frac{2}{\lambda_{1}}\right) I_{n, T}-1\right|>\frac{\epsilon}{2}\right\}+P\left\{\left(-\frac{2 \theta}{T} \frac{2}{\lambda_{1}}\right)\left|I_{m, n, T}-I_{n, T}\right|>\frac{\epsilon}{2}\right\} \\
& \leq C \exp \left(\frac{T \theta}{16} \epsilon^{2}\right)+\frac{16 \theta^{2}}{T^{2}} \frac{E\left|I_{m, n, T}-I_{n, T}\right|^{2}}{\epsilon^{2}} \\
& \leq C \exp \left(\frac{T \theta}{16} \epsilon^{2}\right)+C \frac{T^{2} / n^{2}}{\epsilon^{2}} \text {. }
\end{aligned}
$$


Here the bound for the first term in (3.8) comes from Lemma 1.2.4(a) and that for the second term from Lemma 3.3.2(c). From the proof of Lemma 3.3.2(b) we have

$$
E\left|V_{m, n, T}\right|^{2} \leq C \frac{T^{4}}{n^{2}}
$$

Next

$$
\begin{aligned}
& K_{2}=P\left\{\left|\left(\frac{T}{-2 \theta} \frac{\lambda_{1}}{2}\right)^{1 / 2} \theta \frac{V_{m, n, T}}{I_{m, n, T}}\right|>\epsilon\right\} \\
& =P\left\{\left|\frac{\left(\frac{-2 \theta}{T} \frac{2}{\lambda_{1}}\right)^{1 / 2} \theta V_{m, n, T}}{\left(-\frac{2 \theta}{T} \frac{2}{\lambda_{1}}\right) I_{m, n, T}}\right|>\epsilon\right\} \\
& =P\left\{\left|\left(\frac{-2 \theta}{T} \frac{2}{\lambda_{1}}\right)^{1 / 2} \theta V_{m, n, T}\right|>\delta\right\}+P\left\{\left(-\frac{2 \theta}{T} \frac{2}{\lambda_{1}}\right) I_{m, n, T}<\frac{\delta}{\epsilon}\right\} \\
& \text { (where we choose } \delta=\epsilon-C \epsilon^{2} \text { ) } \\
& \leq P\left\{\left|\left(\frac{-2 \theta}{T} \frac{2}{\lambda_{1}}\right)^{1 / 2} \theta V_{m, n, T}\right|>\delta\right\}+P\left\{\left|\left(-\frac{2 \theta}{T} \frac{2}{\lambda_{1}}\right) I_{m, n, T}-1\right|>\delta_{1}\right\} \\
& \text { (where } \delta_{1}=\frac{\epsilon-\delta}{\delta}=C \epsilon \text { ) } \\
& \leq-\frac{2 \theta}{T} \theta^{2} \frac{E\left|V_{m, n, T}\right|^{2}}{\delta^{2}}+C \exp \left(\frac{T \theta}{16} \delta_{1}^{2}\right)+C \frac{T^{2} / n^{2}}{\delta_{1}^{2}} \\
& \leq C \frac{T^{3} / n^{2}}{\delta^{2}}+C \exp \left(\frac{T \theta}{16} \delta_{1}^{2}\right)+C \frac{T^{2} / n^{2}}{\delta_{1}^{2}} \text { (by (3.9) and (3.8)). }
\end{aligned}
$$

Now combining bounds from $J_{1}, J_{2}, K_{1}$ and $K_{2}$, we have since $T / n \rightarrow 0$

$$
\begin{aligned}
& \sup _{x \in \mathbb{R}}\left|P\left\{\left(\frac{T}{-2 \theta} \frac{\lambda_{1}}{2}\right)^{1 / 2}\left(\hat{\theta}_{m, n, T}-\theta\right) \leq x\right\}-\Phi(x)\right| \\
\leq & C T^{-1 / 2}+C \exp \left(\frac{T \theta}{16} \epsilon^{2}\right)+C \frac{T / n}{\epsilon^{2}}+C \frac{T^{2} / n^{2}}{\epsilon^{2}}+C \frac{T^{3} / n^{2}}{\delta^{2}} \\
& +C \exp \left(\frac{T \theta}{16} \delta_{1}^{2}\right)+C\left(\frac{T^{2} / n^{2}}{\delta_{1}^{2}}\right)+\epsilon .
\end{aligned}
$$

Choosing $\epsilon=C T^{-1 / 2}(\log T)^{1 / 2}, C$ large, the terms of (3.11) are of the order $O\left(\max \left(T^{-1 / 2}(\log T)^{1 / 2}, \frac{T^{2}}{n}(\log T)^{-1}, \frac{T^{4}}{n^{2}}(\log T)^{-1}\right)\right)$. This proves (a).

(b) Using the expression (3.3), we have

$$
\begin{aligned}
& \sup _{x \in \mathbb{R}}\left|P\left\{I_{m, n, T}^{1 / 2}\left(\hat{\theta}_{m, n, T}-\theta\right) \leq x\right\}-\Phi(x)\right| \\
= & \sup _{x \in \mathbb{R}}\left|P\left\{\frac{Y_{m, n, T}}{I_{m, n, T}^{1 / 2}}+\theta \frac{V_{m, n, T}}{I_{m, n, T}^{1 / 2}} \leq x\right\}-\Phi(x)\right| \\
\leq & \sup _{x \in \mathbb{R}}\left|P\left\{\frac{Y_{m, n, T}}{I_{m, n, T}^{1 / 2}} \leq x\right\}-\Phi(x)\right|+P\left\{\left|\theta \frac{V_{m, n, T}}{I_{m, n, T}^{1 / 2}}\right|>\epsilon\right\}+\epsilon . \\
= & : \quad H_{1}+H_{2}+\epsilon .
\end{aligned}
$$


Note that

$$
\begin{aligned}
H_{1} & =\sup _{x \in \mathbb{R}}\left|P\left\{\frac{Y_{m, n, T}-Y_{n, T}+Y_{n, T}}{I_{m, n}^{1 / 2}} \leq x\right\}-\Phi(x)\right| \\
& =\sup _{x \in \mathbb{R}}\left|P\left\{\frac{Y_{n, T}}{I_{m, n, T}^{1 / 2}} \leq x\right\}-\Phi(x)\right|+P\left\{\frac{\left|Y_{m, n, T}-Y_{n, T}\right|}{I_{m, n, T}^{1 / 2}}>\epsilon\right\}+\epsilon . \\
=: & F_{1}+F_{2}+\epsilon .
\end{aligned}
$$

Now

$$
\begin{aligned}
F_{1}= & \sup _{x \in \mathbb{R}}\left|P\left\{\frac{Y_{n, T}}{I_{m, n, T}^{1 / 2}} \leq x\right\}-\Phi(x)\right| \\
\leq & \sup _{x \in \mathbb{R}}\left|P\left\{\left(\frac{-2 \theta}{T} \frac{2}{\lambda_{1}}\right)^{1 / 2} Y_{n, T} \leq x\right\}-\Phi(x)\right| \\
& +P\left\{\left|\left(\frac{-2 \theta}{T} \frac{2}{\lambda_{1}}\right)^{1 / 2} I_{m, n, T}^{1 / 2}-1\right|>\epsilon\right\}+\epsilon \text { (by Lemma 1.2.1(b)) } \\
\leq & C_{T}^{-1 / 2}+P\left\{\left|\left(\frac{-2 \theta}{T}\right) I_{m, n, T}-1\right|>\epsilon\right\}+\epsilon \text { (byCorollary 1.2.3(a)) } \\
\leq & C T^{-1 / 2}+C \exp \left(-\frac{T \theta}{16} \epsilon^{2}\right)+C \frac{T^{2} / n^{2}}{\epsilon^{2}}+\epsilon . \text { (by (3.8)) }
\end{aligned}
$$

On the other hand,

$$
\begin{aligned}
F_{2} \leq & P\left\{\frac{\left|Y_{m, n, T}-Y_{n, T}\right|}{\left.I_{m, n, T}^{1 / 2}>\epsilon\right\}}\right. \\
\leq & P\left\{\left(\frac{-2 \theta}{T} \frac{2}{\lambda_{1}}\right)^{1 / 2}\left|Y_{m, n, T}-Y_{n, T}\right|>\delta\right\}+P\left\{\left|\left(\frac{-2 \theta}{T} \frac{2}{\lambda_{1}}\right)^{1 / 2} I_{m, n, T}^{1 / 2}-1\right|>\delta_{1}\right\} \\
& \left(\text { where } \delta=\epsilon-C \epsilon^{2} \text { and } \delta_{1}=(\epsilon-\delta) / \epsilon>0\right) \\
\leq & \frac{\left(\frac{-2 \theta}{T} \frac{2}{\lambda_{1}}\right) E\left|Y_{m, n, T}-Y_{n, T}\right|^{2}}{\delta^{2}}+P\left\{\left|\left(\frac{-2 \theta}{T}\right) I_{m, n, T}-1\right|>\delta_{1}\right\} \\
\leq & C \frac{T / n}{\delta^{2}}+C \exp \left(\frac{T \theta}{16} \delta_{1}^{2}\right)+C \frac{T^{2} / n^{2}}{\delta_{1}^{2}} \text { (from Lemma 3.3.2(a) and (3.8).) }
\end{aligned}
$$

Using (3.15) and (3.14) in (3.13), we obtain

$$
\begin{aligned}
H_{1}= & \sup _{x \in \mathbb{R}}\left|P\left\{\frac{Y_{n, T}}{I_{m, n, T}^{1 / 2}} \leq x\right\}-\Phi(x)\right| \\
\leq & C T^{-1 / 2}+C \exp \left(\frac{T \theta}{16} \epsilon^{2}\right)+C \frac{T / n}{\delta^{2}}+C \frac{T^{2} / n^{2}}{\delta_{1}^{2}} \\
& +C \exp \left(\frac{T \theta}{16} \delta_{1}^{2}\right)+C \frac{T^{2} / n^{2}}{\epsilon^{2}}+\epsilon .
\end{aligned}
$$




$$
\begin{aligned}
H_{2}= & P\left\{\left|\theta \frac{V_{m, n, T}}{I_{m, n, T}^{1 / 2}}\right|>\epsilon\right\} \\
= & P\left\{\frac{\left|\left(\frac{-2 \theta}{T} \frac{2}{\lambda_{1}}\right)^{1 / 2} \theta V_{m, n, T}\right|}{\left|\left(\frac{-2 \theta}{T} \frac{2}{\lambda_{1}}\right)^{1 / 2} I_{m, n, T}^{1 / 2}\right|}>\epsilon\right\} \\
\leq & P\left\{\left|\left(\frac{-2 \theta}{T} \frac{2}{\lambda_{1}}\right)^{1 / 2} \theta V_{m, n, T}\right|>\delta\right\}+P\left\{\left|\left(\frac{-2 \theta}{T} \frac{2}{\lambda_{1}}\right)^{1 / 2} I_{m, n, T}^{1 / 2}\right|<\delta / \epsilon\right\} \\
\leq & \left(-\frac{2 \theta}{T} \frac{2}{\lambda_{1}}\right) \theta^{2} \frac{E\left|V_{m, n, T}\right|^{2}}{\delta^{2}}+P\left\{\left|\left(-\frac{2 \theta}{T} \frac{2}{\lambda_{1}}\right) I_{m, n, T}-1\right|>\delta_{1}\right\} \\
& \left(\text { where } 0<\delta<\epsilon \text { and } \delta_{1}=(\epsilon-\delta) / \epsilon=C \epsilon>0\right) \\
\leq & C \frac{T^{3} / n^{2}}{\delta^{2}}+C \exp \left(\frac{T \theta}{16} \delta_{1}^{2}\right)+C \frac{T^{2} / n^{2}}{\delta_{1}^{2}} \text {. (from (3.9) and (3.8)) }
\end{aligned}
$$

Using (3.17) and (3.16) in (3.12) and choosing $\epsilon=C T^{-1 / 2}(\log T)^{1 / 2}, C$ large, the terms of (3.12) are of the order $O\left(\max \left(T^{-1 / 2}(\log T)^{1 / 2}, \frac{T^{2}}{n}(\log T)^{-1}, \frac{T^{4}}{n^{2}}(\log T)^{-1}\right)\right)$. This proves (b).

(c) Let $D_{m, n, T}=\left\{\left|\hat{\theta}_{m, n, T}-\theta\right| \leq d_{T}\right\}$ and $d_{T}=C T^{-1 / 2}(\log T)^{1 / 2}$.

On the set $D_{m, n, T}$, expanding $\left(2\left|\theta_{m, n, T}\right|\right)^{-1 / 2}$, we obtain

$$
\begin{aligned}
\left(-2 \hat{\theta}_{m, n, T}\right)^{-1 / 2} & =(-2 \theta)^{-1 / 2}\left[1-\frac{\theta-\hat{\theta}_{m, n, T}}{\theta}\right]^{-1 / 2} \\
& =(-2 \theta)^{-1 / 2}\left[1+\frac{1}{2}\left(\frac{\theta-\hat{\theta}_{m, n, T}}{\theta}\right)+O\left(d_{T}^{2}\right)\right] .
\end{aligned}
$$

Then

$$
\begin{aligned}
\sup _{x \in \mathbb{R}}\left|P\left\{\left(\frac{T}{2\left|\hat{\theta}_{m, n, T}\right|} \frac{\lambda_{1}}{2}\right)^{1 / 2}\left(\hat{\theta}_{m, n, T}-\theta\right) \leq x\right\}-\Phi(x)\right| \\
\leq \sup _{x \in \mathbb{R}}\left|P\left\{\left(\frac{T}{2\left|\hat{\theta}_{m, n, T}\right|} \frac{\lambda_{1}}{2}\right)^{1 / 2}\left(\hat{\theta}_{m, n, T}-\theta\right) \leq x, D_{m, n, T}\right\}-\Phi(x)\right|+P\left(D_{m, n, T}^{c}\right) \\
\\
=P\left(D_{m, n, T}^{c}\right) \\
=\quad P\left\{\left|\hat{\theta}_{m, n, T}-\theta\right|>C T^{-1 / 2}(\log T)^{1 / 2}\right\} \\
=\quad P\left\{\left(\frac{T}{-2 \theta} \frac{\lambda_{1}}{2}\right)^{1 / 2}\left|\hat{\theta}_{m, n, T}-\theta\right|>C(\log T)^{1 / 2}(-2 \theta)^{-1 / 2}\right\} \\
\leq \quad C\left(\max \left(T^{-1 / 2}(\log T)^{1 / 2}, \frac{T^{2}}{n}(\log T)^{-1}, \frac{T^{4}}{n^{2}}(\log T)^{-1}\right)\right. \\
\quad+2\left(1-\Phi\left((\log T)^{1 / 2}(-2 \theta)^{-1 / 2}\right)(\mathrm{by} \operatorname{Theorem} 5.3 .3(\mathrm{a}))\right. \\
\leq \quad C\left(\max \left(T^{-1 / 2}(\log T)^{1 / 2}, \frac{T^{2}}{n}(\log T)^{-1}, \frac{T^{4}}{n^{2}}(\log T)^{-1}\right)\right) .
\end{aligned}
$$


On the set $D_{m, n, T}$,

$$
\left|\left(\frac{\hat{\theta}_{m, n, T}}{\theta}\right)^{1 / 2}-1\right| \leq C T^{-1 / 2}(\log T)^{1 / 2}
$$

Hence upon choosing $\epsilon=C T^{-1 / 2}(\log T)^{1 / 2}, C$ large we obtain

$$
\begin{aligned}
& \quad\left|P\left\{\left(\frac{T}{-2 \hat{\theta}_{m, n, T}} \frac{\lambda_{1}}{2}\right)^{1 / 2}\left(\hat{\theta}_{m, n, T}-\theta\right) \leq x, D_{m, n, T}\right\}-\Phi(x)\right| \\
& \leq\left|P\left\{\left(\frac{T}{-2 \theta} \frac{\lambda_{1}}{2}\right)^{1 / 2}\left(\hat{\theta}_{m, n, T}-\theta\right) \leq x, D_{m, n, T}\right\}-\Phi(x)\right| \\
& +P\left\{\left|\left(\frac{\hat{\theta}_{m, n, T}}{\theta}\right)^{1 / 2}-1\right|>\epsilon, D_{T}\right\}+\epsilon
\end{aligned}
$$

(by Lemma 1.2.1(b))

$$
\leq C\left(\max \left(T^{-1 / 2}(\log T)^{1 / 2}, \frac{T^{2}}{n}(\log T)^{-1}, \frac{T^{4}}{n^{2}}(\log T)^{-1}\right)\right)
$$

(by Theorem 3.3.3(a)).

(c) follows from (3.18) - (3.20).

\section{Theorem 3.1.2}

$$
\sup _{x \in \mathbb{R}}\left|P\left\{I_{m, n, T}\left(-\frac{2 \theta}{T}\right)^{1 / 2}\left(\hat{\theta}_{m, n, T}-\theta\right) \leq x\right\}-\Phi(x)\right|=O\left(T^{-1 / 2} \bigvee\left(\frac{T n}{m}\right)^{1 / 3}\right)
$$

Proof : Let $\quad a_{m, n, T}:=Z_{m, n, T}-Z_{n, T}, \quad b_{m, n, T}:=I_{m, n, T}-I_{n, T}$.

$$
\text { By Lemma 5.3.2 } E\left|a_{m, n, T}\right|^{2}=O\left(\frac{T^{2}}{n}\right) \text { and } E\left|b_{m, n, T}\right|^{2}=O\left(\frac{T^{4}}{n^{2}}\right) \text {. }
$$

From (3.5), we have

$$
\begin{aligned}
I_{m, n, T} \hat{\theta}_{m, n, T} & =\sum_{i=1}^{n} u_{k}\left(t_{i-1}\right)\left[u_{k}\left(t_{i}\right)-u_{k}\left(t_{i-1}\right)\right] \\
& =\int_{0}^{T} u_{k}(t) d u_{k}(t)+a_{m, n, T} \\
& =\int_{0}^{T} u_{k}(t) d W_{k}(t)+\theta \int_{0}^{T} u_{k}^{2}(t) d t+a_{m, n, T} .
\end{aligned}
$$

Hence $I_{m, n, T}\left(\hat{\theta}_{m, n, T}-\theta\right)=-\theta b_{m, n, T}+a_{m, n, T}$. 
Thus

$$
\begin{aligned}
& \sup _{x \in \mathbb{R}}\left|P\left\{I_{m, n, T}\left(-\frac{2 \theta}{T} \frac{2}{\lambda_{1}}\right)^{1 / 2}\left(\hat{\theta}_{m, n, T}-\theta\right) \leq x\right\}-\Phi(x)\right| \\
= & \sup _{x \in \mathbb{R}}\left|P\left\{\left(-\frac{2 \theta}{T} \frac{2}{\lambda_{1}}\right)^{1 / 2}\left[Y_{n, T}-\theta b_{m, n, T}+a_{m, n, T}\right] \leq x\right\}-\Phi(x)\right| \\
\leq & \sup _{x \in \mathbb{R}}\left|P\left\{\left(-\frac{2 \theta}{T} \frac{2}{\lambda_{1}}\right)^{1 / 2} Y_{n, T} \leq x\right\}-\Phi(x)\right| \\
& +P\left\{\left|\left(-\frac{2 \theta}{T} \frac{2}{\lambda_{1}}\right)^{1 / 2}\left[-\theta b_{m, n, T}+a_{m, n, T}\right]\right|>\epsilon\right\}+\epsilon \\
\leq & C T^{-1 / 2}+\left(-\frac{2 \theta}{T} \frac{2}{\lambda_{1}}\right) \frac{E\left|-\theta b_{m, n, T}+a_{n, T}\right|^{2}}{\epsilon^{2}}+\epsilon \\
\leq & C T^{-1 / 2}+C \frac{T / n}{\epsilon^{2}}+\epsilon \text { (by Corollary 1.2.3(a) and (3.21)). }
\end{aligned}
$$

Choosing $\epsilon=\left(\frac{T}{n}\right)^{1 / 3}$, the rate is $O\left(T^{-1 / 2} \bigvee\left(\frac{T}{n}\right)^{1 / 3}\right)$

Theorem 3.1.3

$$
\left|\hat{\theta}_{m, n, T}-\theta_{n, T}\right|=O_{P}\left(\frac{T^{2}}{n}\right)^{1 / 2}
$$

Proof: Note that

$$
\hat{\theta}_{m, n, T}-\theta_{n, T}=\frac{Z_{m, n, T}}{I_{m, n, T}}-\frac{Z_{n, T}}{I_{n, T}} .
$$

From Lemma 3.2 it follows that $\left|Z_{m, n, T}-Z_{n, T}\right|=O_{P}\left(\frac{T^{2}}{n}\right)^{1 / 2}$ and $\left|I_{m, n, T}-I_{n, T}\right|=O_{P}\left(\frac{T^{4}}{n^{2}}\right)^{1 / 2}$. Now the theorem follows easily from the from the Lemma 5.2.1.

\subsection{Berry-Esseen Type Bounds for AMLE1}

Theorem 3.2.1 Let $\beta_{n, T}=O\left(T^{-1 / 2}(\log T)^{1 / 2} \bigvee \frac{T^{4} n^{3}}{m^{2}}(\log T)^{-1}\right)$.

$$
\begin{aligned}
& \text { (a) } \sup _{x \in \mathbb{R}}\left|P\left\{\left(-\frac{T}{2 \theta} \frac{\lambda_{1}}{2}\right)^{1 / 2}\left(\hat{\theta}_{m, n, T, 1}-\theta\right) \leq x\right\}-\Phi(x)\right|=O\left(\beta_{n, T}\right), \\
& \text { (b) } \sup _{x \in \mathbb{R}}\left|P\left\{I_{n, T}^{1 / 2}\left(\hat{\theta}_{m, n, T, 1}-\theta\right) \leq x\right\}-\Phi(x)\right|=O\left(\beta_{n, T}\right), \\
& \text { (c) } \sup _{x \in \mathbb{R}}\left|P\left\{\left(\frac{T}{2\left|\hat{\theta}_{m, n, T, 1}\right|} \frac{\lambda_{1}}{2}\right)^{1 / 2}\left(\hat{\theta}_{m, n, T, 1}-\theta\right) \leq x\right\}-\Phi(x)\right|=O\left(\beta_{n, T}\right) .
\end{aligned}
$$

Proof. (a) From (1.8), we have 


$$
\begin{aligned}
I_{m, n, T} \hat{\theta}_{m, n, T, 1} & =\frac{1}{2}\left(u_{k}^{2}(T)-T\right) \\
& =\int_{0}^{T} u_{k}(t) d u_{k}(t) \\
& =\int_{0}^{T} u_{k}(t) d W_{k}(t)+\theta \int_{0}^{T} u_{k}(t)^{2} d t \\
& =Y_{n, T}+\theta I_{n, T} .
\end{aligned}
$$

Thus

$$
\begin{aligned}
& \left(-\frac{T}{2 \theta} \frac{\lambda_{1}}{2}\right)^{1 / 2}\left(\hat{\theta}_{m, n, T, 1}-\theta\right) \\
= & \frac{\left(-\frac{T}{2 \theta}\right)^{1 / 2} Y_{T}+\theta\left(-\frac{T}{2 \theta} \frac{\lambda_{1}}{2}\right)^{1 / 2}\left(I_{n, T}-I_{m, n, T}\right)}{I_{n, T}} \\
= & \frac{\left(-\frac{2 \theta}{T} \frac{2}{\lambda_{1}}\right)^{1 / 2} Y_{n, T}+\left(-\frac{2 \theta}{T} \frac{2}{\lambda_{1}}\right)^{1 / 2}\left(I_{n, T}-I_{m, n, T}\right)}{\left(-\frac{2 \theta}{T}\right) I_{n, T}} .
\end{aligned}
$$

Now

$$
\begin{aligned}
& \sup _{x \in \mathbb{R}}\left|P\left\{\left(-\frac{T}{2 \theta} \frac{\lambda_{1}}{2}\right)^{1 / 2}\left(\hat{\theta}_{m, n, T, 1}-\theta\right) \leq x\right\}-\Phi(x)\right| \\
& =\sup _{x \in \mathbb{R}} P\left\{\frac{\left(-\frac{2 \theta}{T} \frac{2}{\lambda_{1}}\right)^{1 / 2} Y_{n, T}+\left(-\frac{2 \theta}{T}\right)^{1 / 2}\left(I_{n, T}-I_{m, n, T}\right)}{\left(-\frac{2 \theta}{T} \frac{2}{\lambda_{1}}\right) I_{m, n, T}} \leq x\right\}-\Phi(x) \mid \\
& \leq \sup _{x \in \mathbb{R}}\left|P\left\{\left(-\frac{2 \theta}{T} \frac{2}{\lambda_{1}}\right)^{1 / 2} Y_{n, T} \leq x\right\}-\Phi(x)\right| \\
& +P\left\{\left|\theta\left(-\frac{2 \theta}{T} \frac{2}{\lambda_{1}}\right)^{1 / 2}\left(I_{m, n, T}-I_{n, T}\right)\right|>\epsilon\right\}+P\left\{\left|\left(-\frac{2 \theta}{T} \frac{2}{\lambda_{1}}\right) I_{m, n, T}-1\right|>\epsilon\right\}+2 \epsilon \\
& \leq C T^{-1 / 2}+\theta^{2} \frac{\left(-\frac{2 \theta}{T} \frac{2}{\lambda_{1}}\right) E\left|I_{m, n, T}-I_{n, T}\right|^{2}}{\epsilon^{2}}+C \exp \left(\frac{T \theta}{4} \epsilon^{2}\right)+C \frac{T^{2}}{n^{2} \epsilon^{2}}+2 \epsilon
\end{aligned}
$$

(The bound for the 3rd term in the r.h.s. of (4.2) is from (3.8).)

$$
\leq C T^{-1 / 2}+C \frac{T^{3}}{n^{2} \epsilon^{2}}+C \exp \left(\frac{T \theta}{4} \epsilon^{2}\right)+C \frac{T^{2}}{n^{2} \epsilon^{2}}+\epsilon
$$

(by Lemma 3.3.2 (c))

Choosing $\epsilon=C T^{-1 / 2}(\log T)^{1 / 2}$, the terms in the r.h.s. of (4.3) are of the order $O\left(T^{-1 / 2}(\log T)^{1 / 2} \bigvee \frac{T^{4}}{n^{2}}(\log T)^{-1}\right)$.

(b) From (4.1), we have

$$
I_{m, n, T}^{1 / 2}\left(\hat{\theta}_{m, n, T, 1}-\theta\right)=\frac{Y_{n, T}+\theta\left(I_{n, T}-I_{m, n, T}\right)}{I_{m, n, T}^{1 / 2}} .
$$


Now

$$
\begin{aligned}
& \sup _{x \in \mathbb{R}}\left|P\left\{I_{m, n, T}^{1 / 2}\left(\hat{\theta}_{m, n, T, 1}-\theta\right) \leq x\right\}-\Phi(x)\right| \\
= & \sup _{x \in \mathbb{R}}\left|P\left\{\frac{Y_{n, T}}{I_{m, n, T}^{1 / 2}}+\theta \frac{I_{n, T}-I_{m, n, T}}{I_{m, n, T}^{1 / 2}} \leq x\right\}-\Phi(x)\right| \\
\leq & \sup _{x \in \mathbb{R}}\left|P\left\{\frac{Y_{n, T}}{I_{m, n, T}^{1 / 2}} \leq x\right\}-\Phi(x)\right|+P\left\{\left|\frac{\theta\left(I_{n, T}-I_{m, n, T}\right)}{I_{m, n, T}^{1 / 2}}\right|>\epsilon\right\}+\epsilon \\
=: & U_{1}+U_{2}+\epsilon .
\end{aligned}
$$

We have from (3.8),

$$
U_{1} \leq C T^{-1 / 2}+C \exp \left(\frac{T \theta}{16} \epsilon^{2}\right)+C \frac{T^{2}}{n^{2} \epsilon^{2}}+\epsilon
$$

Now

$$
\begin{aligned}
U_{2}= & P\left\{|\theta| \frac{\left.\left|I_{m, n, T}-I_{n, T}\right|>\epsilon\right\}}{I_{m, n, T}^{1 / 2} \mid}\right\} \\
= & P\left\{|\theta| \frac{\left(-\frac{2 \theta}{T} \frac{2}{\lambda_{1}}\right)^{1 / 2}\left(I_{m, n, T}-I_{n, T}\right) \mid}{\left|\left(-\frac{2 \theta}{T} \frac{2}{\lambda_{1}}\right)^{1 / 2} I_{m, n, T}^{1 / 2}\right|}>\epsilon\right\} \\
\leq & P\left\{\left|\left(-\frac{2 \theta}{T} \frac{2}{\lambda_{1}}\right)^{1 / 2}\right|\left|I_{m, n, T}-I_{n, T}\right|>\delta\right\}+P\left\{\left|\left(-\frac{2 \theta}{T}\right)^{1 / 2} I_{m, n, T}^{1 / 2}-1\right|>\delta_{1}\right\} \\
\leq & \left(-\frac{2 \theta}{T} \frac{2}{\lambda_{1}}\right) \frac{E\left|I_{m, n, T}-I_{n, T}\right|^{2}}{\delta^{2}}+P\left\{\left|\left(-\frac{2 \theta}{T}\right) I_{m, n, T}-1\right|>\delta_{1}\right\} \\
\leq & C \frac{T^{3}}{n^{2} \delta^{2}}+C \exp \left(\frac{T \theta}{16} \delta_{1}^{2}\right)+C \frac{T^{2} / n^{2}}{\delta_{1}^{2}} .
\end{aligned}
$$

Here the bound for the first term in the r.h.s. of (4.6) comes from Lemma 3.3.2(c) and that for the second term is from $J_{2}$ in (3.8).

Now using the bounds (4.5) and (4.7) in (4.4) with $\epsilon=C T^{-1 / 2}(\log T)^{1 / 2}$, we obtain that the terms in (4.4) is of the order $O\left(T^{-1 / 2}(\log T)^{1 / 2} \bigvee \frac{T^{4}}{n^{2}}(\log T)^{-1}\right)$.

(c) Let $G_{m, n, T}:=\left\{\left|\hat{\theta}_{m, n, T, 1}-\theta\right| \leq d_{T}\right\}$ and $d_{T}=C T^{-1 / 2}(\log T)^{1 / 2}$. On the set $G_{T}$, expanding $\left(2\left|\theta_{m, n, T, 1}\right|\right)^{1 / 2}$, we obtain,

$$
\begin{aligned}
\left(-2 \hat{\theta}_{m, n, T, 1}\right)^{-1 / 2} & =(-2 \theta)^{1 / 2}\left[1-\frac{\theta-\hat{\theta}_{m, n, T, 1}}{\theta}\right]^{-1 / 2} \\
& =(-2 \theta)^{1 / 2}\left[1+\frac{1}{2}\left(\frac{\theta-\hat{\theta}_{m, n, T, 1}}{\theta}\right)+O\left(d_{T}^{2}\right)\right] .
\end{aligned}
$$

Then

$$
\begin{aligned}
& \sup _{x \in \mathbb{R}}\left|P\left\{\left(\frac{T}{2\left|\hat{\theta}_{m, n, T, 1}\right|} \frac{\lambda_{1}}{2}\right)^{1 / 2}\left(\hat{\theta}_{m, n, T, 1}-\theta\right) \leq x\right\}-\Phi(x)\right| \\
\leq & \sup _{x \in \mathbb{R}}\left\{P\left(\frac{T}{2\left|\hat{\theta}_{m, n, T, 1}\right|}\right)^{1 / 2}\left(\hat{\theta}_{m, n, T, 1}-\theta\right) \leq x, G_{T}\right\}+P\left(G_{T}^{c}\right) .
\end{aligned}
$$


Now

$$
\begin{aligned}
& P\left(G_{m, n, T}^{c}\right) \\
= & P\left\{\left|\hat{\theta}_{m, n, T, 1}-\theta\right|>C T^{-1 / 2}(\log T)^{1 / 2}\right\} \\
= & P\left\{\left(-\frac{T}{2 \theta} \frac{\lambda_{1}}{2}\right)^{1 / 2}\left|\hat{\theta}_{m, n, T, 1}-\theta\right|>C(\log T)^{1 / 2}(-2 \theta)^{-1 / 2}\right\} \\
\leq & C\left(T^{-1 / 2}(\log T)^{1 / 2} \bigvee \frac{T^{4}}{n^{2}}(\log T)^{-1}\right)+2\left(1-\Phi \log T^{1 / 2}(-2 \theta)^{-1 / 2}\right)
\end{aligned}
$$

(by Theorem 5.4.1(a))

$\leq C\left(T^{-1 / 2}(\log T)^{1 / 2} \bigvee \frac{T^{4}}{n^{2}}(\log T)^{-1}\right)$.

On the set $G_{T}$,

$$
\left|\left(\frac{\hat{\theta}_{m, n, T, 1}}{\theta}\right)^{1 / 2}-1\right| \leq C T^{-1 / 2}(\log T)^{1 / 2}
$$

Hence upon choosing $\epsilon=C T^{-1 / 2}(\log T)^{1 / 2}, C$ large

$$
\begin{aligned}
& \left|P\left\{\left(\frac{T}{-2 \theta_{m, n, T, 1}}\right)^{1 / 2}\left(\hat{\theta}_{m, n, T, 1}-\theta\right) \leq x, G_{m, n, T}\right\}-\Phi(x)\right| \\
\leq & \mid P\left\{\left(\frac{T}{-2 \theta}\right)^{1 / 2}\left(\hat{\theta}_{m, n, T, 1}-\theta\right) \leq x, G_{m, n, T}\right\}+P\left\{\left|\left(\frac{\hat{\theta}_{m, n, T, 1}}{\theta}\right)^{1 / 2}-1\right|>\epsilon, G_{m, n, T}\right\}+\epsilon
\end{aligned}
$$

(by Lemma 1.2.1 (b))

$\leq C\left(T^{-1 / 2}(\log T)^{1 / 2} \bigvee \frac{T^{4}}{n^{2}}(\log T)^{-1}\right) \quad($ by Theorem 3.4.1(a)).

\section{Theorem 3.2.2}

$$
\sup _{x \in \mathbb{R}}\left|P\left\{I_{m, n, T}\left(-\frac{2 \theta}{T} \frac{2}{\lambda_{1}}\right)^{1 / 2}\left(\hat{\theta}_{m, n, T, 1}-\theta\right) \leq x\right\}-\Phi(x)\right|=O\left(T^{-1 / 2} \bigvee\left(\frac{T^{3} n^{2}}{m^{2}}\right)^{1 / 3}\right)
$$

Proof: From (4.1) we have

$$
I_{n, T}\left(-\frac{2 \theta}{T} \frac{2}{\lambda_{1}}\right)^{1 / 2}\left(\hat{\theta}_{m, n, T, 1}-\theta\right)=\left(-\frac{2 \theta}{T} \frac{2}{\lambda_{1}}\right)^{1 / 2} Y_{n, T}+\theta\left(-\frac{2 \theta}{T}\right)^{1 / 2} \frac{2}{\lambda_{1}}\left(I_{n, T}-I_{m, n, T}\right) .
$$

Hence by Corollary 1.2.3(a) and Lemma 3.3.2(c)

$$
\begin{aligned}
& \sup _{x \in \mathbb{R}}\left|P\left\{I_{n, T}\left(-\frac{2 \theta}{T} \frac{2}{\lambda_{1}}\right)^{1 / 2}\left(\hat{\theta}_{m, n, T, 1}-\theta\right) \leq x\right\}-\Phi(x)\right| \\
= & \sup _{x \in \mathbb{R}}\left|P\left\{\left(-\frac{2 \theta}{T} \frac{2}{\lambda_{1}}\right)^{1 / 2} Y_{n, T}+\theta\left(-\frac{2 \theta}{T} \frac{2}{\lambda_{1}}\right)^{1 / 2}\left(I_{n, T}-I_{m, n, T}\right) \leq x\right\}-\Phi(x)\right| \\
\leq & \sup _{x \in \mathbb{R}}\left|P\left\{\left(-\frac{2 \theta}{T}\right)^{1 / 2} Y_{n, T} \leq x\right\}-\Phi(x)\right|+P\left\{\left|\theta\left(-\frac{2 \theta}{T} \frac{2}{\lambda_{1}}\right)^{1 / 2}\left(I_{n, T}-I_{m, n, T}\right)\right|>\epsilon\right\}+\epsilon . \\
\leq & C T^{-1 / 2}+C \frac{E\left|I_{n, T}-I_{m, n, T}\right|^{2}}{T \epsilon^{2}}+\epsilon \\
\leq & C T^{-1 / 2}+C \frac{T^{3} n^{3}}{m^{2} \epsilon^{2}}+\epsilon
\end{aligned}
$$


Choosing $\epsilon=\left(\frac{T^{3} n^{3}}{m^{2}}\right)^{1 / 3}$, the theorem follows.

Theorem 3.2.3 $\left|\hat{\theta}_{m, n, T, 1}-\theta_{T}\right|=O_{P}\left(\frac{T^{2} n}{m}\right)$.

Proof. We have from (2.3) $\theta_{n, T}=Z_{n, T} / I_{n, T}$. By Itô formula it is easy to see that

$$
\theta_{m, n, T, 1}=Z_{m, n, T} / I_{m, n, T}
$$

Hence applying Lemma 3.3.7 with the aid of Lemma 3.3.2(c) the theorem follows.

\subsection{Berry-Essen Bounds for AMCEs}

The following theorem gives the bound on the error of normal approximation of the AMCE. Note that part (a) uses parameter dependent nonrandom norming. While this is useful for testing hypotheses about $\theta$, it may not necessarily give a confidence interval. The normings in parts (b) and (c) are sample dependent which can be used for obtaining a confidence interval. Following theorem shows that asymptotic normality of the AMCEs need $T \rightarrow \infty$ and $\frac{T}{\sqrt{n}} \rightarrow 0$.

Theorem 3.3.1 Denote $b_{m, n, T}:=O\left(\max \left(T^{-1 / 2}(\log T)^{1 / 2},\left(\frac{T^{4}}{n^{2}}\right)(\log T)^{-1}\right)\right)$.
(a) $\sup _{x \in \mathbb{R}}\left|P\left\{\left(-\frac{T}{2 \theta} \frac{\lambda_{1}}{2}\right)^{1 / 2}\left(\tilde{\theta}_{m, n, T}-\theta\right) \leq x\right\}-\Phi(x)\right|=O\left(b_{m, n, T}\right)$,
(b) $\sup _{x \in \mathbb{R}}\left|P\left\{I_{m, n, T}^{1 / 2}\left(\tilde{\theta}_{m, n, T}-\theta\right) \leq x\right\}-\Phi(x)\right|=O\left(b_{m, n, T}\right)$,
(c) $\sup _{x \in \mathbb{R}}\left|P\left\{\left(\frac{T}{2\left|\tilde{\theta}_{m, n, T}\right|} \frac{\lambda_{1}}{2}\right)^{1 / 2}\left(\tilde{\theta}_{m, n, T}-\theta\right) \leq x\right\}-\Phi(x)\right|=O\left(b_{m, n, T}\right)$.

Proof (a) Observe that

$$
\left(-\frac{T}{2 \theta} \frac{\lambda_{1}}{2}\right)^{1 / 2}\left(\tilde{\theta}_{m, n, T}-\theta\right)=\frac{\left(-\frac{2 \theta}{T} \frac{2}{\lambda_{1}}\right)^{1 / 2} Y_{n, T}}{\left(-\frac{2 \theta}{T} \frac{2}{\lambda_{1}}\right) I_{m, n, T}}
$$

where

$$
Y_{n, T}:=-\theta I_{n, T}-\frac{T}{2} \quad \text { and } \quad I_{m, n, T}:=\int_{0}^{T} u_{k}(t)^{2} d t
$$

Thus, we have

$$
I_{m, n, T} \tilde{\theta}_{m, n, T}=-\frac{T}{2}=Y_{n, T}+\theta I_{m, n, T}
$$


Hence,

$$
\begin{aligned}
& \left(-\frac{T}{2 \theta} \frac{\lambda_{1}}{2}\right)^{1 / 2}\left(\tilde{\theta}_{m, n, T}-\theta\right) \\
= & \frac{\left(-\frac{T}{2 \theta} \frac{\lambda_{1}}{2}\right)^{1 / 2} Y_{n, T}+\theta\left(-\frac{T}{2 \theta}\right)^{1 / 2}\left(I_{n, T}-I_{m, n, T}\right)}{I_{m, n, T}} \\
= & \frac{\left(-\frac{2 \theta}{T} \frac{2}{\lambda_{1}}\right)^{1 / 2} Y_{n, T}+\left(-\frac{2 \theta}{T} \frac{2}{\lambda_{1}}\right)^{1 / 2}\left(I_{n, T}-I_{m, n, T}\right)}{\left(-\frac{2 \theta}{T}\right) I_{m, n, T}}
\end{aligned}
$$

Further,

$$
\begin{aligned}
& P\left\{\left|\left(\frac{-2 \theta}{T} \frac{2}{\lambda_{1}}\right)\left(I_{m, n, T}-1\right)\right|>\epsilon\right\} \\
= & \left\{\left|\left(\frac{-2 \theta}{T} \frac{2}{\lambda_{1}}\right)\left(I_{m, n, T}-I_{n, T}+I_{n, T}\right)-1\right|>\epsilon\right\} \\
\leq & P\left\{\left|\left(\frac{-2 \theta}{T} \frac{2}{\lambda_{1}}\right) I_{n, T}-1\right|>\frac{\epsilon}{2}\right\}+P\left\{\left(-\frac{2 \theta}{T} \frac{2}{\lambda_{1}}\right)\left|I_{m, n, T}-I_{n, T}\right|>\frac{\epsilon}{2}\right\} \\
\leq & C \exp \left(\frac{T \theta}{16} \epsilon^{2}\right)+\frac{16 \theta^{2}}{T^{2}} \frac{E\left|I_{m, n, T}-I_{n, T}\right|^{2}}{\epsilon^{2}} \\
\leq & C \exp \left(\frac{T \theta}{16} \epsilon^{2}\right)+C \frac{T^{2} / n^{2}}{\epsilon^{2}} .
\end{aligned}
$$

Next, observe that

$$
\begin{aligned}
& \sup _{x \in \mathbb{R}}\left|P\left\{\left(-\frac{T}{2 \theta} \frac{\lambda_{1}}{2}\right)^{1 / 2}\left(\tilde{\theta}_{m, n, T}-\theta\right) \leq x\right\}-\Phi(x)\right| \\
= & \sup _{x \in \mathbb{R}}\left|P\left\{\frac{\left(-\frac{2 \theta}{T} \frac{2}{\lambda_{1}}\right)^{1 / 2} Y_{n, T}+\left(-\frac{2 \theta}{T} \frac{2}{\lambda_{1}}\right)^{1 / 2}\left(I_{n, T}-I_{m, n, T}\right)}{\left(-\frac{2 \theta}{T} \frac{2}{\lambda_{1}}\right) I_{m, n, T}} \leq x\right\}-\Phi(x)\right| \\
\leq & \sup _{x \in \mathbb{R}}\left|P\left\{\left(-\frac{2 \theta}{T} \frac{2}{\lambda_{1}}\right)^{1 / 2} Y_{n, T} \leq x\right\}-\Phi(x)\right| \\
& +P\left\{\left|\theta\left(-\frac{2 \theta}{T} \frac{2}{\lambda_{1}}\right)^{1 / 2}\left(I_{m, n, T}-I_{T}\right)\right|>\epsilon\right\}+P\left\{\left|\left(-\frac{2 \theta}{T} \frac{2}{\lambda_{1}}\right) I_{m, n, T}-1\right|>\epsilon\right\}+2 \epsilon \\
\leq & C T^{-1 / 2}+\theta^{2} \frac{\left(-\frac{2 \theta}{T} \frac{2}{\lambda_{1}}\right) E\left|I_{m, n, T}-I_{n, T}\right|^{2}}{\epsilon^{2}}+C \exp \left(\frac{T \theta}{4} \epsilon^{2}\right)+C \frac{T^{2}}{n^{2} \epsilon^{2}}+2 \epsilon,
\end{aligned}
$$

(the bound for the $3^{\text {rd }}$ term in the right hand side of (2.4) is obtained from (2.3))

$\leq C T^{-1 / 2}+C \frac{T^{2}}{n^{2} \epsilon^{2}}+C \exp \left(\frac{T \theta}{4} \epsilon^{2}\right)+C \frac{T}{n^{2} \epsilon^{2}}+\epsilon$

(by Lemma 2.3(a)). 
Choosing $\epsilon=C T^{-1 / 2}(\log T)^{1 / 2}$, the terms in the right hand side of (2.5) are of the order $O\left(\max \left(T^{-1 / 2}(\log T)^{1 / 2},\left(\frac{T^{4}}{n^{2}}\right)(\log T)^{-1}\right)\right)$.

(b) From (2.1), we have

$$
I_{m, n, T}^{1 / 2}\left(\tilde{\theta}_{m, n, T}-\theta\right)=\frac{Y_{n, T}+\theta\left(I_{n, T}-I_{m, n, T}\right)}{I_{m, n, T}^{1 / 2}} .
$$

Then,

$$
\begin{aligned}
& \sup _{x \in \mathbb{R}}\left|P\left\{I_{m, n, T}^{1 / 2}\left(\tilde{\theta}_{m, n, T}-\theta\right) \leq x\right\}-\Phi(x)\right| \\
= & \sup _{x \in \mathbb{R}}\left|P\left\{\frac{Y_{n, T}}{I_{m, n, T}^{1 / 2}}+\theta \frac{I_{n, T}-I_{m, n, T}}{I_{m, n, T}^{1 / 2}} \leq x\right\}-\Phi(x)\right| \\
\leq & \sup _{x \in \mathbb{R}}\left|P\left\{\frac{Y_{n, T}}{I_{m, n, T}^{1 / 2}} \leq x\right\}-\Phi(x)\right|+P\left\{\left|\frac{\theta\left(I_{n, T}-I_{m, n, T}\right)}{I_{m, n, T}^{1 / 2}}\right|>\epsilon\right\}+\epsilon \\
= & : \quad U_{1}+U_{2}+\epsilon .
\end{aligned}
$$

We have from (2.3),

$$
U_{1} \leq C T^{-1 / 2}+C \exp \left(\frac{T \theta}{16} \epsilon^{2}\right)+C \frac{T^{2}}{n^{2} \epsilon^{2}}+\epsilon .
$$

Now,

$$
\begin{aligned}
U_{2} & =P\left\{|\theta| \frac{I_{m, n, T}-I_{n, T}}{I_{n, T}^{1 / 2}} \mid>\epsilon\right\}=P\left\{|\theta| \frac{\left|\left(-\frac{2 \theta}{T}\right)^{1 / 2}\left(I_{m, n, T}-I_{n, T}\right)\right|}{\left|\left(-\frac{2 \theta}{T} \frac{2}{\lambda_{1}}\right)^{1 / 2} I_{m, n, T}^{1 / 2}\right|}>\epsilon\right\} \\
& \leq P\left\{\left|\left(-\frac{2 \theta}{T} \frac{2}{\lambda_{1}}\right)^{1 / 2}\right|\left|I_{m, n, T}-I_{n, T}\right|>\delta\right\}+P\left\{\left|\left(-\frac{2 \theta}{T} \frac{2}{\lambda_{1}}\right)^{1 / 2} I_{m, n, T}^{1 / 2}-1\right|>\delta_{1}\right\}
\end{aligned}
$$
(where $\delta=\epsilon-C \epsilon^{2}$ and $\delta_{1}=(\epsilon-\delta) / \epsilon>0$ )

$$
\begin{aligned}
& \leq\left(-\frac{2 \theta}{T}\right) \frac{E\left|I_{m, n, T}-I_{n, T}\right|^{2}}{\delta^{2}}+P\left\{\left|\left(-\frac{2 \theta}{T} \frac{2}{\lambda_{1}}\right) I_{m, n, T}-1\right|>\delta_{1}\right\} \\
& \leq C \frac{T^{3}}{n^{2} \delta^{2}}+C \exp \left(\frac{T \theta}{16} \delta_{1}^{2}\right)+C \frac{T^{2}}{n^{2} \delta_{1}^{2}} .
\end{aligned}
$$

Here, the bound for the first term in the right hand side of (2.7) comes from Lemma 2.2(c) and that for the second term is obtained from (2.3).

Now, using the bounds (2.7) and (2.9) in (2.6) with $\epsilon=C T^{-1 / 2}(\log T)^{1 / 2}$, we obtain that the terms in (2.6) are of the order $O\left(\max \left(T^{-1 / 2}(\log T)^{1 / 2},\left(\frac{T^{4}}{n^{2}}\right)(\log T)^{-1}\right)\right)$.

(c) Let

$$
G_{m, n, T}:=\left\{\left|\tilde{\theta}_{m, n, T}-\theta\right| \leq d_{T}\right\}, \quad \text { and } \quad d_{T}:=C T^{-1 / 2}(\log T)^{1 / 2}
$$


On the set $G_{T}$, expanding $\left(2\left|\tilde{\theta}_{m, n, T}\right|\right)^{1 / 2}$, we obtain

$$
\begin{aligned}
& \left(-2 \tilde{\theta}_{m, n, T}\right)^{-1 / 2}=(-2 \theta)^{1 / 2}\left[1-\frac{\theta-\tilde{\theta}_{m, n, T}}{\theta}\right]^{-1 / 2} \\
& =(-2 \theta)^{1 / 2}\left[1+\frac{1}{2}\left(\frac{\theta-\tilde{\theta}_{m, n, T}}{\theta}\right)\right]+O\left(d_{T}^{2}\right) .
\end{aligned}
$$

Then,

$$
\begin{aligned}
& \sup _{x \in \mathbb{R}}\left|P\left\{\left(\frac{T}{2\left|\tilde{\theta}_{m, n, T}\right|}\right)^{1 / 2}\left(\tilde{\theta}_{m, n, T}-\theta\right) \leq x\right\}-\Phi(x)\right| \\
\leq & \sup _{x \in \mathbb{R}}\left\{P\left(\frac{T}{2\left|\tilde{\theta}_{m, n, T}\right|}\right)^{1 / 2}\left(\tilde{\theta}_{m, n, T}-\theta\right) \leq x, G_{m, n, T}\right\}+P\left(G_{m, n, T}^{c}\right) .
\end{aligned}
$$

Now,

$$
\begin{aligned}
P\left(G_{m, n, T}^{c}\right) & =P\left\{\left|\tilde{\theta}_{m, n, T}-\theta\right|>C T^{-1 / 2}(\log T)^{1 / 2}\right\} \\
& =P\left\{\left(-\frac{T}{2 \theta} \frac{\lambda_{1}}{2}\right)^{1 / 2}\left|\tilde{\theta}_{m, n, T}-\theta\right|>C(\log T)^{1 / 2}(-2 \theta)^{-1 / 2}\right\} \\
& \leq C \max \left(T^{-1 / 2}(\log T)^{1 / 2}, \frac{T^{3}}{n^{2}}(\log T)^{-1}\right)+2\left(1-\Phi \log T^{1 / 2}(-2 \theta)^{-1 / 2}\right)
\end{aligned}
$$

(by Theorem 2.1(a))

$$
\leq C \max \left(T^{-1 / 2}(\log T)^{1 / 2}, \frac{T^{3}}{n^{2}}(\log T)^{-1}\right) \text {. }
$$

On the set $G_{T}$,

$$
\left|\left(\frac{\tilde{\theta}_{m, n, T}}{\theta}\right)^{1 / 2}-1\right| \leq C T^{-1 / 2}(\log T)^{1 / 2} .
$$

Hence, upon choosing $\epsilon=C T^{-1 / 2}(\log T)^{1 / 2}, C$ large, we obtain

$$
\begin{aligned}
& \left|P\left\{\left(\frac{T}{-2 \tilde{\theta}_{m, n, T}}\right)^{1 / 2}\left(\tilde{\theta}_{m, n, T}-\theta\right) \leq x, G_{m, n, T}\right\}-\Phi(x)\right| \\
\leq & \left|P\left\{\left(\frac{T}{-2 \theta}\right)^{1 / 2}\left(\tilde{\theta}_{m, n, T}-\theta\right) \leq x, G_{m, n, T}\right\}\right|+P\left\{\left|\left(\frac{\tilde{\theta}_{m, n, T}}{\theta}\right)^{1 / 2}-1\right|>\epsilon, G_{m, n, T}\right\}+\epsilon
\end{aligned}
$$

(by Lemma 1.1(b))

$$
\leq C \max \left(T^{-1 / 2}(\log T)^{1 / 2}, \frac{T^{4}}{n^{2}}(\log T)^{-1}\right)
$$

(by Theorem 2.1(a)).

In the following theorem, we improve the bound on the error of normal approximation using a mixture of random and nonrandom normings. Thus asymptotic normality of the AMCEs need 
$T \rightarrow \infty$ and $\frac{T}{n^{2 / 3}} \rightarrow 0$ which are sharper than the bound in Theorem 2.1.

Theorem 3.3.2

$$
\sup _{x \in \mathbb{R}}\left|P\left\{I_{m, n, T}\left(-\frac{2 \theta}{T} \frac{2}{\lambda_{1}}\right)^{1 / 2}\left(\tilde{\theta}_{m, n, T}-\theta\right) \leq x\right\}-\Phi(x)\right|=O\left(\max \left(T^{-1 / 2},\left(\frac{T^{3}}{n^{2}}\right)^{1 / 3}\right)\right) .
$$

Proof From (2.2), we have

$$
I_{m, n, T}\left(-\frac{2 \theta}{T}\right)^{1 / 2}\left(\tilde{\theta}_{m, n, T}-\theta\right)=\left(-\frac{2 \theta}{T} \frac{2}{\lambda_{1}}\right)^{1 / 2} Y_{n, T}+\theta\left(-\frac{2 \theta}{T}\right)^{1 / 2}\left(I_{n, T}-I_{m, n, T}\right) .
$$

Hence, by Lemma 2.1-2.3

$$
\begin{aligned}
& \sup _{x \in \mathbb{R}}\left|P\left\{I_{m, n, T}\left(-\frac{2 \theta}{T} \frac{2}{\lambda_{1}}\right)^{1 / 2}\left(\tilde{\theta}_{m, n, T}-\theta\right) \leq x\right\}-\Phi(x)\right| \\
= & \sup _{x \in \mathbb{R}}\left|P\left\{\left(-\frac{2 \theta}{T} \frac{2}{\lambda_{1}}\right)^{1 / 2} Y_{n, T}+\theta\left(-\frac{2 \theta}{T}\right)^{1 / 2}\left(I_{n, T}-I_{m, n, T}\right) \leq x\right\}-\Phi(x)\right| \\
\leq & \sup _{x \in R}\left|P\left\{\left(-\frac{2 \theta}{T}\right)^{1 / 2} Y_{n, T} \leq x\right\}-\Phi(x)\right|+P\left\{\left|\theta\left(-\frac{2 \theta}{T} \frac{2}{\lambda_{1}}\right)^{1 / 2}\left(I_{n, T}-I_{m, n, T}\right)\right|>\epsilon\right\}+\epsilon \\
\leq & C T^{-1 / 2}+C \frac{E\left|I_{n, T}-I_{m, n, T}\right|^{2}}{T \epsilon^{2}}+\epsilon \leq C T^{-1 / 2}+C \frac{T^{3}}{n^{2} \epsilon^{2}}+\epsilon .
\end{aligned}
$$

Choosing $\epsilon=\left(\frac{T^{3}}{n^{2}}\right)^{1 / 3}$, the theorem follows.

The following theorem gives stochastic bound on the error of approximation of the continuous MCE by AMCEs.

Theorem 3.3.3

$$
\begin{aligned}
& \text { (a) }\left|\tilde{\theta}_{m, n, T}-\tilde{\theta}_{n, T}\right|=O_{P}\left(\frac{T}{n}\right)^{1 / 2} . \\
& \text { (b) }\left|\tilde{\theta}_{m, n, T, 1}-\tilde{\theta}_{n, T}\right|=O_{P}\left(\frac{T^{2}}{n^{2}}\right)^{1 / 2} .
\end{aligned}
$$

Proof From (1.9) and (1.14), we have

$$
\tilde{\theta}_{n, T}=-\frac{T}{2 I_{n, T}}, \quad \tilde{\theta}_{m, n, T}=-\frac{T}{2 I_{m, n, T}} .
$$

Hence, applying Lemma 1.2 with the aid of Lemma 2.3(a) and noting that $\left|\frac{I_{m, n, T}}{T}\right|=O_{P}(1)$ and $\left|\frac{I_{n, T}}{T}\right|=O_{P}(1)$ the part (a) of theorem follows.

From (1.9) and (1.16), we have

$$
\tilde{\theta}_{n, T}=-\frac{T}{2 I_{n, T}}, \quad \tilde{\theta}_{m, n, T, 1}=-\frac{T}{I_{m, n, T}+J_{m, n, T}} .
$$

Applying Lemma 1.2 with the aid of Lemma 2.3(b) and noting that $\left|\frac{J_{m, n, T}}{T}\right|=O_{P}(1)$ and $\left|\frac{I_{n, T}}{T}\right|=O_{P}(1)$ the part (b) of theorem follows. 


\subsection{First Order Asymptotic Theory}

As a consequence of the above three sub-sections we obtain the consistency and asymptotic normality of the AMLE, AMLE1, AMCE and AMCE1:

\section{Theorem 3.4.1}

a) $\hat{\theta}_{m, n, T} \rightarrow \theta$ in probability as $n \rightarrow \infty$ and $m \rightarrow \infty$ such that $n / m^{2} \rightarrow 0$.

b) $n\left(\hat{\theta}_{m, n, T}-\theta\right) \rightarrow^{\mathcal{D}} \eta$ as $n \rightarrow \infty$ and $m \rightarrow \infty$ such that $n^{3} / m^{2} \rightarrow 0$

where $\eta$ is a normal random variable with mean zero and variance $\frac{2 \theta(\alpha-\gamma+1)^{2}}{\lambda_{1} T(\alpha-\gamma+1 / 2)}$.

\section{Theorem 3.4.2}

a) $\hat{\theta}_{m, n, T, 1} \rightarrow \theta$ in probability as $n \rightarrow \infty$ and $m \rightarrow \infty$ such that $n / m^{2} \rightarrow 0$.

b) $n\left(\hat{\theta}_{m, n, T, 1}-\theta\right) \rightarrow^{\mathcal{D}} \eta$ as $n \rightarrow \infty$ and $m \rightarrow \infty$ such that $n^{3} / m^{2} \rightarrow 0$ where $\eta$ is a normal random variable with mean zero and variance $\frac{2 \theta(\alpha-\gamma+1)^{2}}{\lambda_{1} T(\alpha-\gamma+1 / 2)}$.

\section{Theorem 3.4.3}

a) $\tilde{\theta}_{m, n, T} \rightarrow \theta$ in probability as $n \rightarrow \infty$ and $m \rightarrow \infty$ such that $n / m^{2} \rightarrow 0$.

b) $n\left(\tilde{\theta}_{m, n, T}-\theta\right) \rightarrow^{\mathcal{D}} \eta$ as $n \rightarrow \infty$ and $m \rightarrow \infty$ such that $n^{3} / m^{2} \rightarrow 0$ where $\eta$ is a normal random variable with mean zero and variance $\frac{2 \theta(\alpha-\gamma+1)^{2}}{\lambda_{1} T(\alpha-\gamma+1 / 2)}$.

\section{Theorem 3.4.4}

a) $\tilde{\theta}_{m, n, T, 1} \rightarrow \theta$ in probability as $n \rightarrow \infty$ and $m \rightarrow \infty$ such that $n / m^{2} \rightarrow 0$.

b) $n\left(\tilde{\theta}_{m, n, T, 1}-\theta\right) \rightarrow^{\mathcal{D}} \eta$ as $n \rightarrow \infty$ and $m \rightarrow \infty$ such that $n^{3} / m^{2} \rightarrow 0$ where $\eta$ is a normal random variable with mean zero and variance $\frac{2 \theta(\alpha-\gamma+1)^{2}}{\lambda_{1} T(\alpha-\gamma+1 / 2)}$.

\section{Hypothesis Testing from Continuous Observation}

We estimate and control the Type I and Type II errors of a simple hypothesis testing problem of the viscosity coefficient $\theta$ of the SNS equation (1.1). We study a class of rejection regions and provide thresholds that guarantee that the statistical errors are smaller than the given upper bound. The tests are of likelihood ratio type. The proofs are based on the large deviation bounds.

The problem is to test the null hypothesis $\mathcal{H}_{0}: \theta=\theta_{0}$ versus the alternative hypothesis $\mathcal{H}_{1}$ : $\theta=\theta_{1}$ based on continuous observation $U_{n}^{T}$ where $\theta_{0} \neq \theta_{1}$. 
Without loss of generality we will assume that $\theta_{1}>\theta_{0}$. We fix the level of significance $\alpha \in(0,1)$. Suppose $\left.R \in \mathcal{B}\left(C[0, T] ; \mathbb{R}^{n}\right)\right)$ is a rejection region for the test, i.e, if $U_{n}^{T} \in R$, we reject the null hypothesis and accept the alternative hypothesis. The quantity $P_{\theta_{0}}^{n, T}(R)$ is called the Type I error of the test $R$ and $1-P_{\theta_{1}}^{n, T}(R)$ is called the Type II error. Naturally we seek rejection region with Type I error $P_{\theta_{0}}^{n, T}(R)$ smaller than the significance level $\alpha$ and thus we consider the following class of rejection regions:

$$
\left.\mathcal{K}_{\alpha}:=\left\{R \in \mathcal{B}\left(C[0, T] ; \mathbb{R}^{n}\right)\right): P_{\theta_{0}}^{n, T}(R) \leq \alpha\right\} .
$$

Consider the test of the form

$$
R_{n}=\left\{U_{n}^{T}: \ln L\left(\theta_{0}, \theta_{1}, U_{T}^{n}\right) \geq \zeta N\right\}
$$

where $\zeta$ is some number depending on $n$ and $T$ and $N:=\sum_{k=1}^{n} \lambda_{k}^{2 \beta}$.

Let

$$
\begin{gathered}
R_{n}^{\#}=\left\{U_{T}^{n}: L\left(\theta_{0}, \theta_{1}, U_{T}^{n}\right) \geq \tilde{c}_{\alpha}(n)\right\}, \\
\left.\tilde{\mathcal{K}}_{\alpha}^{\#}:=\left\{R \in \mathcal{B}\left(C[0, T] ; \mathbb{R}^{n}\right)\right): \lim \sup _{n \rightarrow \infty} \sqrt{N}\left(P_{\theta_{0}}^{n, T}(R)-\alpha\right) \leq \tilde{\alpha}_{1}\right\} .
\end{gathered}
$$

where $\tilde{c}_{\alpha}(n)$ is a constant depending on $n$ and $\alpha$ only and $\tilde{\alpha}_{1}$ is a constant depending on $\alpha$. The probability $P_{\theta_{1}}^{n, T}(R)$ of the true decision under $\mathcal{H}_{1}$ is called the power of the test and the goal is to find the most powerful rejection region $R^{*} \in \mathcal{K}_{\alpha}$ for the observation $U_{n}^{T}$.

We say that the rejection region $R^{*} \in \mathcal{K}_{\alpha}$ is the most powerful (has the smallest Type II error) in the class $\mathcal{K}_{\alpha}$ if

$$
P_{\theta_{1}}^{n, T}(R) \leq P_{\theta_{1}}^{n, T}\left(R^{*}\right), \text { for all } R \in \mathcal{K}_{\alpha} .
$$

The corresponding test is called the most powerful test.

Our main results of this section are the following:

Theorem 4.1 For sufficiently large $n$, under the null hypothesis $\mathcal{H}_{0}$, we have

$$
P_{\theta_{0}}^{n, T}\left(R_{n}^{\#}\right)=\alpha+\alpha_{1} n^{-1}+O\left(n^{-2}\right)
$$

For large enough n, we have

$$
\left|P_{\theta_{0}}^{n, T}\left(R_{n}^{\#}\right)-\alpha\right| \leq C n^{-1}
$$

where $C$ is a constant independent of $n$.

We show that the Type I Error has the following upper bound estimate:

\section{Theorem 4.2}

$$
P_{\theta_{0}}^{n, T}\left(R_{n}^{0}\right) \leq(1+\rho) \alpha
$$

where $\rho$ denotes a given threshold of error tolerance.

We show that the Type II Error has the following upper bound estimate: 


\section{Theorem 4.3}

$$
1-P_{\theta_{1}}^{n, T}\left(R_{n}^{0}\right) \leq(1+\rho) \exp \left(-\frac{\left(\theta_{1}-\theta_{0}\right)^{2}}{16 \theta_{0}} N T\right) .
$$

Let us denote by the rejection region (likelihood ratio test) of the form

$$
R_{n}^{*}=\left\{U_{T}^{n}: L\left(\theta_{0}, \theta_{1}, U_{T}^{n}\right) \geq c_{\alpha}\right\}
$$

where $c_{\alpha} \in \mathbb{R}$ such that $P_{\theta_{1}}^{n, T}\left(L\left(\theta_{0}, \theta_{1}, U_{T}^{n}\right) \geq c_{\alpha}\right)=\alpha$.

As one may expect, once we have an MLE for the parameter of interest $\theta$ as well as its consistency, a Neyman-Pearson type lemma should give an answer to a hypothesis testing problem.

\section{Theorem 4.4 (Neyman-Pearson Lemma)}

Let $c_{\alpha}$ be a real number such that

$$
P_{\theta_{0}}^{n, T}\left(L\left(\theta_{0}, \theta_{1}, U_{T}^{n}\right) \geq c_{\alpha}\right)=\alpha
$$

Then,

$$
R_{n}^{*}=\left\{U_{T}^{n}: L\left(\theta_{0}, \theta_{1}, U_{T}^{n}\right) \geq c_{\alpha}\right\}
$$

is the most powerful rejection region in the class $\mathcal{K}_{\alpha}$.

For a fixed $n \in \mathbb{N}$, let $\mathcal{K}$ be a generic set of rejection regions

$$
\left.\mathcal{K} \subset\left\{\left(R_{n}\right)_{n \in \mathbb{N}}: R_{n} \in \mathcal{B}\left(C[0, T] ; \mathbb{R}^{n}\right)\right)\right\} .
$$

We say that the rejection region $R_{n}^{*}$ is asymptotically most powerful, in the class $\mathcal{K}$, as $n \rightarrow \infty$ if

$$
\liminf _{n \rightarrow \infty} \frac{1-P_{\theta_{1}}^{n, T}\left(R_{n}\right)}{1-P_{\theta_{1}}^{n, T}\left(R_{n}^{*}\right)} \geq 1
$$

for all $R_{n} \in \mathcal{K}$.

We will consider classes of rejection regions that have asymptotically a Type I error close to the significance level $\alpha$. On the other hand, one would like to consider rejection regions such that $P_{\theta_{1}}^{n, T}\left(R_{n}\right) \rightarrow 1$ as $n \rightarrow \infty$. The concept of asymptotically most powerful test depicts to those tests, within the considered class of tests, that have the fastest rate of convergence of their powers to one. $P_{\theta_{1}}^{n, T}\left(R_{n}^{*}\right)$ has the fastest rate of convergence to 1 as $n \rightarrow \infty$

Under $\mathcal{H}_{1}$, the $\mathrm{OU}$ process is

$$
d u_{k}(t)=-\theta_{1} \lambda_{k}^{2 \beta} u_{k}(t) d t+\sigma \lambda_{k}^{-\gamma} d W_{k}(t), u_{k}(0)=0 .
$$

The proof uses the following results: 
Proposition 4.1 Let $\mathbf{u}=\left(u_{1}, u_{2}, \cdots, u_{n}\right) \in \mathbb{R}^{n}$ and $a>0$, we set

$$
\begin{gathered}
r(\mathbf{u}, t):=\frac{a\left(\theta_{1}^{2}-\theta_{0}^{2}\right)}{2 \sigma^{2}} \sum_{k=1}^{n} \lambda_{k}^{4 \beta+2 \gamma} u_{k}^{2}(t), \\
F(\mathbf{u}):=\exp \left(-\frac{a\left(\theta_{1}-\theta_{0}\right)}{2 \sigma^{2}} \sum_{k=1}^{n} \lambda_{k}^{2 \beta+2 \gamma} u_{k}^{2}+\frac{a\left(\theta_{1}-\theta_{0}\right)}{2} N T\right)
\end{gathered}
$$

By the Feynman-Kac formula, the function

$$
f(\mathbf{u}, t)=E_{\theta_{1}}\left[\exp \left(-\int_{t}^{T} r\left(U_{n}^{s}, s\right) d s\right) F\left(U_{n}^{T}\right) \mid U_{n}^{t}=\mathbf{u}\right]
$$

is the only solution to the PDE

$$
f_{t}+\frac{\sigma^{2}}{2} \sum_{k=1}^{n} \lambda_{k}^{-2 \gamma} f_{u_{k} u_{k}}-\theta_{1} \sum_{k=1}^{n} \lambda_{k}^{2 \beta} u_{k} f_{u_{k}}=r(\mathbf{u}, t) f, \quad f(\mathbf{u}, T)=F(\mathbf{u}) .
$$

The solution of this PDE is

$$
\begin{aligned}
f(\mathbf{u}, t)=\exp \left(\sum_{k=1}^{n} \frac{\alpha_{k} u_{k}^{2}\left[\sinh \left(\gamma_{k} t\right)+\beta_{k} \cosh \left(\gamma_{k} t\right)\right]}{\cosh \left(\gamma_{k} t\right)+\beta_{k} \sinh \left(\gamma_{k} t\right)}+\frac{1}{2} \sum_{k=1}^{n} \ln \left|\frac{\cosh \left(\gamma_{k} T\right)+\beta_{k} \sinh \left(\gamma_{k} T\right)}{\cosh \left(\gamma_{k} t\right)+\beta_{k} \sinh \left(\gamma_{k} t\right)}\right|\right. \\
\left.+\frac{\theta_{1}}{2 \sigma^{2}} \sum_{k=1}^{n} \lambda_{k}^{2 \beta+2 \gamma} u_{k}^{2}+\frac{a\left(\theta_{1}-\theta_{0}\right)+\theta_{1}}{2} N T-\frac{\theta_{1}}{2} N t\right)
\end{aligned}
$$

where

$$
\begin{gathered}
\alpha_{k}=-\frac{1}{2 \sigma^{2}}\left(a\left(\theta_{1}^{2}-\theta_{0}^{2}\right)+\theta_{1}^{2}\right)^{1 / 2} \lambda_{k}^{2 \beta+2 \gamma}, \beta_{k}=\frac{p \cosh \left(\gamma_{k} T\right)-\sinh \left(\gamma_{k} T\right)}{\cosh \left(\gamma_{k} T\right)-p \sinh \left(\gamma_{k} T\right)} \\
\gamma_{k}=-\left(a\left(\theta_{1}^{2}-\theta_{0}^{2}\right)+\theta_{1}^{2}\right)^{1 / 2} \lambda_{k}^{2 \beta}, p=\frac{a\left(\theta_{1}-\theta_{0}\right)+\theta_{1}}{\left(a\left(\theta_{1}^{2}-\theta_{0}^{2}\right)+\theta_{1}^{2}\right)^{1 / 2}} .
\end{gathered}
$$

By taking $\mathbf{u}=t=0$, we obtain

$$
\begin{gathered}
g_{T}(a)=E\left[\exp \left(a \ln L\left(\theta_{0}, \theta_{1}, U_{n}^{T}\right)\right)\right]=f(0,0) \\
=\exp \left[-\frac{1}{2} \sum_{k=1}^{n} \ln \left(\cosh \left(\gamma_{k} T\right)-p \sinh \left(\gamma_{k} T\right)\right)+\frac{a\left(\theta_{1}-\theta_{0}\right)+\theta_{1}}{2} N T\right] .
\end{gathered}
$$

Note that

$$
c(a):=\lim _{T \rightarrow \infty} \ln g_{T}(a)=\left[-\left(a\left(\theta_{1}^{2}-\theta_{0}^{2}\right)+\theta_{1}^{2}\right)^{1 / 2}+a\left(\theta_{1}-\theta_{0}\right)+\theta_{1}\right] \frac{N}{2} .
$$

Let the cumulant generating function of the log-likelihood ratio be given by

$$
g_{T}(a):=E\left[\exp \left(a \ln L\left(\theta_{1}, \theta_{2}, U_{n}^{T}\right)\right)\right] .
$$

By Itô's formula, the log-likelihood ratio is given by

$$
\left.\ln L\left(\theta_{1}, \theta_{2}, U_{n}^{T}\right)\right)=-\frac{\theta_{1}-\theta_{0}}{2 \sigma^{2}} \sum_{k=1}^{n} \lambda_{k}^{2 \beta+2 \gamma} u_{k}^{2}(T)+\frac{\theta_{1}-\theta_{0}}{2} N T-\frac{\theta_{1}^{2}-\theta_{0}^{2}}{2 \sigma^{2}} \sum_{k=1}^{n} \int_{0}^{T} \lambda_{k}^{4 \beta+2 \gamma} u_{k}^{2}(t) d t \text {. }
$$

We prove large deviations as $n \rightarrow \infty$. 
Let

$$
\mathcal{L}_{n}(a):=\frac{1}{N} \ln E\left[\exp \left(a \ln L\left(\theta_{0}, \theta_{1}, U_{n}^{T}\right)\right)\right]
$$

\section{Proposition 4.2}

The log-likelihood function satisfies

$$
\begin{gathered}
\lim _{n \rightarrow \infty} \frac{1}{n^{2}} \ln P\left[\left(n^{-2} \ln L\left(\theta_{0}, \theta_{1}, U_{n}^{T}\right)\right) \geq \eta\right]=-I(\eta), \quad \eta \in\left(\frac{\left(\theta_{1}-\theta_{0}\right)^{2}}{4 \theta_{1}} N, \frac{\theta_{1}-\theta_{0}}{2} N\right), \\
\lim _{n \rightarrow \infty} \frac{1}{n^{2}} \ln P\left[\left(n^{-2} \ln L\left(\theta_{0}, \theta_{1}, U_{n}^{T}\right)\right) \leq \eta\right]=-I(\eta), \quad \eta \in\left(-\infty, \frac{\left(\theta_{1}-\theta_{0}\right)^{2}}{4 \theta_{1}} N\right)
\end{gathered}
$$

where I is the Legender-Fenchel transform

$$
\begin{gathered}
I(\eta):=\sup _{a>a_{-}}(a \eta-c(a))=-\frac{\left(4 \theta_{1} \eta-\left(\theta_{1}-\theta_{0}\right)^{2} N\right)^{2}}{8\left(2 \eta-\left(\theta_{1}-\theta_{0}\right) N\right)\left(\theta_{1}^{2}-\theta_{0}^{2}\right)}, \quad \eta<\frac{\theta_{1}-\theta_{0}}{2} N, \\
I(\eta)=+\infty, \quad \eta \geq \frac{\theta_{1}-\theta_{0}}{2} N
\end{gathered}
$$

with

$$
c(a):=\lim _{n \rightarrow \infty} \frac{1}{n} \ln g_{T}(a), \quad a_{-}=-\frac{\theta_{1}^{2}}{\theta_{1}^{2}-\theta_{0}^{2}}, \quad a_{+}=+\infty .
$$

$c(a)$ is proper and convex with

$$
\eta_{-}=-\infty, \eta_{0}=\frac{\left(\theta_{1}-\theta_{0}\right)^{2}}{4 \theta_{1}} N, \eta_{+}=\frac{\left(\theta_{1}-\theta_{0}\right)}{2} N .
$$

Using the above results, we obtain the exponential rate of convergence of the power of the test.

Theorem 4.5

$$
P_{\theta_{1}}^{n, T}\left(R_{n}^{\#}\right)=1-\exp \left(-\frac{\left(\theta_{1}-\theta_{0}\right)^{2}}{4 \theta_{0}} N T+o(T)\right)
$$

\section{CLT for the Log-Likelihood Ratio Process (LLRP)}

\section{Lemma 4.1}

$$
\frac{1}{N} \frac{\ln L\left(\theta_{0}, \theta_{1}, U_{n}^{T}\right)-\eta N-\mathcal{H}^{\prime}\left(a_{\eta}\right) n}{\zeta_{\eta}} \rightarrow^{\mathcal{D}} \mathcal{N}(0,1) \text { as } n \rightarrow \infty
$$

where

$$
\eta<\frac{\left(\theta_{1}-\theta_{0}\right)^{2} T}{4 \theta_{1}}
$$

and

$$
\zeta_{\eta}^{2}=\frac{\left(-2 \eta+\left(\theta_{1}-\theta_{0}\right) T\right)^{3}}{\left(\theta_{1}^{2}-\theta_{0}^{2}\right) T^{2}}, \quad \mathcal{H}^{\prime}\left(a_{\eta}\right)=-\frac{1}{2} \ln \left(\frac{1}{2}+\frac{1}{2} D(a)\right), \quad D(a)=\frac{\theta_{0}+\left(\theta_{1}-\theta_{0}\right) a}{\sqrt{\theta_{0}^{2}+\left(\theta_{1}^{2}-\theta_{0}^{2}\right) a}} .
$$


Recall that $u_{k}(T), k \geq 1$ are independent. We have the following CLT for stochastic integrals by using CLT for martingales:

\section{Lemma 4.2}

$$
\frac{1}{\sqrt{N}} \sum_{k=1}^{n} \lambda_{k}^{2 \beta+\gamma} \int_{0}^{T} u_{k}(t) d W_{k}(t) \rightarrow^{\mathcal{D}} \mathcal{N}\left(0, \frac{\sigma^{2} T}{2 \theta_{0}}\right) \text { as } n \rightarrow \infty
$$

where $N=\sum_{k=1}^{n} \lambda_{k}^{2 \beta}$.

Let

$$
\xi_{k}:=\lambda_{k}^{2 \beta+\gamma} \int_{0}^{T} u_{k}(t) d W_{k}(t)
$$

Then $E\left(\xi_{k}\right)=0$ and

$$
\operatorname{Var}\left(\xi_{k}\right)=E \xi_{k}^{2}=\lambda_{k}^{4 \beta+2 \gamma} \int_{0}^{T} E u_{k}^{2}(t) d t=\frac{\sigma^{2}}{2 \theta}\left[T \lambda_{k}^{2 \beta}-\frac{1}{2 \theta}\left(1-e^{-2 \theta \lambda_{k}^{2 \beta}} T\right)\right] \sim k^{2 \beta / d}
$$

hence using Lemma 1.2,

$$
\frac{\frac{1}{\sqrt{N}} \sum_{k=1}^{n} \lambda_{k}^{2 \beta+\gamma} \int_{0}^{T} u_{k}(t) d W_{k}(t)}{\left(\frac{1}{N} \sum_{k=1}^{n} \lambda_{k}^{4 \beta+2 \gamma} \int_{0}^{T} E u_{k}^{2}(t) d t\right)^{1 / 2}} \rightarrow^{D} N(0,1) \text { as } n \rightarrow \infty
$$

By basic calculus, we have

$$
\frac{1}{N} \sum_{k=1}^{n} \lambda_{k}^{4 \beta+2 \gamma} \int_{0}^{T} E u_{k}^{2}(t) d t \rightarrow \frac{\sigma^{2} T}{2 \theta_{0}} \text { as } n \rightarrow \infty
$$

Hence the CLT result follows.

We have the following CLT for quadratic variations:

Lemma 4.3

$$
\sqrt{n}\left(\frac{1}{n} \sum_{k=1}^{n} \chi_{k}^{2}-1\right) \rightarrow \mathcal{N}(0,2) \text { as } n \rightarrow \infty
$$

where

$$
\chi_{k}^{2}:=\frac{2 \theta_{0}}{\sigma^{2}\left(1-e^{-2 \theta_{0} \lambda_{k}^{2 \beta} T}\right)} \lambda_{k}^{2 \beta+2 \gamma} u_{k}^{2}(T)
$$

for $k \geq 1$.

Since $u_{k}(T) \sim^{d} \mathcal{N}\left(0, \sigma_{k}^{2}\right)$ where

$$
\sigma_{k}^{-2}=\frac{2 \theta_{0}}{\sigma^{2}\left(1-e^{-2 \theta_{0} \lambda_{k}^{2 \beta} T}\right)} \lambda_{k}^{2 \beta+2 \gamma}
$$

we have $\chi_{k}^{2} \sim^{d} \chi^{2}(1)$ for $k \geq 1$. Since $u_{k}(T)$ are independent, the CLT gives the result. 


\section{Hypothesis Testing from Discrete Observations}

We partition the time interval $[0, T]$ into $n$ equally spaced time intervals $0=t_{0}<t_{1}<\ldots<t_{m}=$ $T$ with $\Delta T=t_{i}-t_{i-1}=T / m$. For each Fourier mode is observed at $m$ time points. We observe the process $U_{T}^{n}$ discretely at $m$ time points. The $n$ Fourier modes are observed at the times points $t_{i}, i=1,2, \ldots, m$. Thus $u_{i, k}, i=1,2, \ldots, m ; k=1,2, \ldots, n$ are the observations. We denote these data by $U_{T}^{m, n}$. Recall that $u_{i, k}:=u_{k}\left(t_{i}\right)$.

The problem is to test the null hypothesis $\mathcal{H}_{0}: \theta=\theta_{0}$ versus the alternative hypothesis $\mathcal{H}_{1}$ : $\theta=\theta_{1}$ based on discrete observations $u_{i, k}, i=1,2, \ldots, m ; k=1,2, \ldots, n$ where $\theta_{0} \neq \theta_{1}$.

Without loss of generality, we will assume that $\theta_{1}>\theta_{0}$. We fix the level of significance $\alpha \in(0,1)$. Suppose that $\left.R \in \mathcal{B}\left(C[0, T] ; \mathbb{R}^{n}\right)\right)$ is a rejection region for the test, i.e, if $U_{T}^{m, n} \in R$, we reject the null hypothesis and accept the alternative hypothesis. Naturally, we seek rejection region with Type I error $P_{\theta_{0}}^{m, n, T}(R)$ smaller than the significance level $\alpha$ and thus we consider the following class of rejection regions:

$$
\left.\mathcal{K}_{\alpha}:=\left\{R \in \mathcal{B}\left(C[0, T] ; \mathbb{R}^{n}\right)\right): P_{\theta_{0}}^{m, n, T}(R) \leq \alpha\right\} .
$$

Consider the test of the form

$$
R_{m, n, T}^{0}=\left\{U_{T}^{m, n}: \ln L\left(\theta_{0}, \theta_{1}, U_{T}^{m, n}\right) \geq \zeta N\right\}
$$

where $\zeta$ is some number depending on $m, n$ and $T$ and $N:=\sum_{k=1}^{n} \lambda_{k}^{2 \beta}$.

The probability $P_{\theta_{1}}^{m, n, T}(R)$ of the true decision under $\mathcal{H}_{1}$ is called the power of the test and the goal is to find the most powerful rejection region $R^{*} \in \mathcal{K}_{\alpha}$ for the observation $U_{n}^{T}$.

We say that the rejection region $R^{*} \in \mathcal{K}_{\alpha}$ is the most powerful (has the smallest Type II error) in the class $\mathcal{K}_{\alpha}$ if

$$
P_{\theta_{1}}^{m, n, T}(R) \leq P_{\theta_{1}}^{m, n, T}\left(R^{*}\right) \text { for all } R \in \mathcal{K}_{\alpha}
$$

Our main results of this section are the following:

\section{Theorem 5.1}

$$
\left|P_{\theta_{0}}^{m, n, T}\left(R_{m, n, T}^{0}\right)-\alpha\right| \leq C n^{-1} m^{-1 / 2}
$$

where $C$ is a constant independent of $n$.

We show that the Type I Error has the following upper bound estimate:

\section{Theorem 5.2}

$$
P_{\theta_{0}}^{m, n, T}\left(R_{m, n, T}^{0}\right) \leq(1+\rho) \alpha .
$$

The Type II Error has the following upper bound estimate: 


\section{Theorem 5.3}

$$
1-P_{\theta_{1}}^{m, n, T}\left(R_{m, n, T}^{0}\right) \leq(1+\rho) \exp \left(-\frac{\left(\theta_{1}-\theta_{0}\right)^{2}}{16 \theta_{0}} N T\right) .
$$

Let us denote by the rejection region (likelihood ratio test) of the form

$$
R_{m, n, T}^{*}=\left\{U_{T}^{m, n}: L\left(\theta_{0}, \theta_{1}, U_{T}^{m, n}\right) \geq c_{\alpha}\right\}
$$

where $c_{\alpha} \in \mathbb{R}$ such that $P_{\theta_{1}}^{m, n, T}\left(L\left(\theta_{0}, \theta_{1}, U_{T}^{m, n}\right) \geq c_{\alpha}\right)=\alpha$.

As one may expect, once we have an AMLE for the parameter of interest $\theta$ as well as its consistency, a Neyman-Pearson type lemma should give an answer to a hypothesis testing problem.

\section{Theorem 5.4 (Neyman-Pearson Lemma)}

Let $c_{\alpha}$ be a real number such that

$$
P_{\theta_{0}}^{m, n, T}\left(L\left(\theta_{0}, \theta_{1}, U_{T}^{m, n}\right) \geq c_{\alpha}\right)=\alpha .
$$

Then

$$
R_{m, n, T}^{*}=\left\{U_{T}^{m, n}: L\left(\theta_{0}, \theta_{1}, U_{T}^{m, n}\right) \geq c_{\alpha}\right\}
$$

is the most powerful rejection region in the class $\mathcal{K}_{\alpha}$.

For fixed $m, n \in \mathbb{N}$, let $\mathcal{K}$ be a generic set of rejection regions

$$
\left.\mathcal{K} \subset\left\{\left(R_{m, n, T}\right)_{m \in \mathbb{N}, n \in \mathbb{N}}: R_{m, n, T} \in \mathcal{B}\left(C[0, T] ; \mathbb{R}^{n}\right)\right)\right\} .
$$

We say that the rejection region $R_{m, n, T}^{*}$ is asymptotically most powerful, in the class $\mathcal{K}$, as $m \rightarrow$ $\infty, n \rightarrow \infty$ if

$$
\liminf _{m \rightarrow \infty, n \rightarrow \infty} \frac{1-P_{\theta_{1}}^{m, n, T}\left(R_{m, n, T}\right)}{1-P_{\theta_{1}}^{m, n, T}\left(R_{m, n, T}^{*}\right)} \geq 1
$$

for all $R_{m, n, T} \in \mathcal{K}$.

We will consider classes of rejection regions that have asymptotically a Type I error close to the significance level $\alpha$. On the other hand, one would like to consider rejection regions such that $P_{\theta_{1}}^{m, n, T}\left(R_{m, n, T}\right) \rightarrow 1$ as $m \rightarrow \infty, n \rightarrow \infty$. The concept of asymptotically most powerful test depicts to those tests, within the considered class of tests, that have the fastest rate of convergence of their powers to one. The probability $P_{\theta_{1}}^{m, n, T}\left(R_{m, n, T}^{*}\right)$ has the fastest rate of convergence to 1 as $m \rightarrow \infty$ and $n \rightarrow \infty$.

Under $\mathcal{H}_{1}$, the OU process is

$$
d u_{k}(t)=-\theta_{1} \lambda_{k}^{2 \beta} u_{k}(t) d t+\sigma \lambda_{k}^{-\gamma} d W_{k}(t), u_{k}(0)=0, \quad k \geq 1 .
$$

Given the tools of proofs of Section 4, the proofs of Section 5 depend on the approximations of integrals including stochastic integrals of Section 3. 


\section{Monte Carlo Method}

In order to simulate the trajectories of the solution (Fourier modes), we discretize the SPDE. Let the Euler-Maruyama scheme of the solution be

$\tilde{u}_{k}^{j}\left(t_{i}\right)=\tilde{u}_{k}^{j}\left(t_{i-1}\right)-\theta \lambda_{k}^{2} \tilde{u}_{k}^{j}\left(t_{i-1}\right) \Delta T+\sigma \lambda_{k}^{-\alpha} \xi_{k, i}^{j}, u_{k}^{j}\left(t_{i}\right)=u_{k}(0), 1 \leq j \leq l, \quad 1 \leq i \leq m \quad 1 \leq k \leq n$

where $\xi_{k, i}^{j}$ are iid Gaussian random variables with zero mean and variance $\Delta T=T / m=t_{i}-$ $t_{i-1}, 1 \leq i \leq m$ and / denotes the number of trials in the Monte Carlo experiment for each Fourier mode. Hence $\tilde{u}_{k}^{j}\left(t_{i}\right)$ is the approximation of $u_{k}^{j}\left(t_{i}\right)$ which is the true value of the $k$-th Fourier mode at time $t_{i}$ of the $j$-th trial in the Monte Carlo Simulation. In what follows, we will investigate how to approximate the Type I and Type II errors of $R^{0}$ test using $\tilde{u}_{k}^{j}\left(t_{i}\right)$ and how the numerical errors are related to $m, I, T$ and $n$. We obtain error estimates of the corresponding Monte Carlo experiments associated with the Euler scheme. We consider $\beta=1$ and $d=1$ with the random forcing term being space time white noise $\gamma=0, \sigma=1$. We assume that $G=[0, \pi]$ and the initial value $U_{0}=0$. In this case $\lambda_{k}=k$. Using the likelihood ratio and Itô formula, we get

$$
\begin{aligned}
& P_{\theta_{0}}^{n, T}\left(R_{T}^{0}\right)=P_{\theta_{0}}^{n, T}\left(\ln L\left(\theta_{0}, \theta_{1}, U_{n}^{T}\right) \geq \eta T\right) \\
& =P_{\theta_{0}}^{n, T}\left(-\sum_{k=1}^{n} \lambda_{k}^{2+2 \alpha}\left(\int_{0}^{T} u_{k}(t) d u_{k}(t)+\frac{\theta_{1}+\theta_{0}}{2 \theta_{0}} \int_{0}^{T} u_{k}(t)\left(\sigma \lambda_{k}^{\gamma} d W_{k}(t)-d u_{k}\right)\right) \geq \frac{\sigma^{2} \eta T}{\theta_{1}-\theta_{0}}\right) \\
& \left.=P_{\theta_{0}}^{n, T}\left(\sum_{k=1}^{n} \lambda_{k}^{2+2 \alpha}\left(\frac{\theta_{1}-\theta_{0}}{2} \int_{0}^{T} u_{k}^{2}(T)-\sigma^{2} \lambda_{k}^{-2 \gamma} T\right)+\left(\theta_{1}+\theta_{0}\right) \int_{0}^{T} u_{k}(t)\left(\sigma \lambda_{k}^{\gamma} d W_{k}(t)\right)\right) \geq \frac{2 \theta_{0} \sigma^{2} \eta T}{\theta_{1}-\theta_{0}}\right) \\
& =P_{\theta_{0}}^{n, T}\left(\frac{\left(\theta_{1}-\theta_{0}\right)}{2 \sigma\left(\theta_{1}+\theta_{0}\right) \sqrt{T}} X_{T}-Y_{T} / \sqrt{T} \geq \frac{2 \theta_{0} \sigma \Delta \eta \sqrt{T}}{\theta_{1}^{2}-\theta_{0}^{2}}\right)
\end{aligned}
$$

where

$$
\begin{gathered}
\eta:=-\frac{\left(\theta_{1}-\theta_{0}\right)^{2}}{4 \theta_{0}} N+\frac{\left(\theta_{1}-\theta_{0}\right)^{2}}{2 \theta_{0}^{2} T}+\frac{\left(\theta_{1}-\theta_{0}\right)^{2}}{2 \theta_{0}^{2}} \sqrt{-\theta_{0} N T^{-1} \ln \alpha+T^{-2} \ln ^{2} \alpha}, \\
\Delta \eta:=\eta+\frac{\left(\theta_{1}-\theta_{0}\right)^{2}}{4 \theta_{0}} N, \quad X_{T}:=\sum_{k=1}^{n} \lambda_{k}^{2+2 \alpha} u_{k}^{2}(T), \quad Y_{T}:=\sum_{k=1}^{n} \lambda_{k}^{2+\alpha} \int_{0}^{T} u_{k}(t) d W_{k}(t) .
\end{gathered}
$$

We approximate $X_{T}$ and $Y_{T}$ by

$$
\tilde{X}_{n, T}^{j}:=\sum_{k=1}^{n} \lambda_{k}^{2+2 \alpha} \tilde{u}_{k}^{j}\left(t_{n}\right)^{2}, \quad \tilde{Y}_{n, T}^{j}:=\sum_{k=1}^{n} \lambda_{k}^{2+\alpha} \sum_{i=1}^{m} \tilde{u}_{k}^{j}\left(t_{i-1}\right) \xi_{k, i}^{j}
$$

respectively. Define

$$
\tilde{R}_{m, T}^{0, j}:=\left\{\frac{\theta_{1}-\theta_{0}}{2 \sigma\left(\theta_{1}+\theta_{0}\right) \sqrt{N}} \tilde{X}_{m, T}^{j}-\tilde{Y}_{m, T}^{j} / \sqrt{N} \geq \frac{2 \theta_{0} \sigma \Delta \eta}{\theta_{1}^{2}-\theta_{0}^{2}} \sqrt{N}\right\} .
$$

The approximation of $P_{\theta_{0}}^{n, T}\left(R_{n, T}^{0}\right)$ is given by

$$
\tilde{P}_{\theta_{0}}^{l, m, n, T}\left(R_{T}^{0}\right)=\frac{1}{l} \sum_{j=1}^{l} I_{\tilde{R}_{m, T}^{0, j}} .
$$


The following theorem gives error estimate on the Monte Carlo simulations:

\section{Theorem 6.1}

$$
\left|\widetilde{P}_{\theta_{0}}^{l, m, n, T}\left(R_{l, n, T}^{0}\right)-P_{\theta_{0}}^{n, T}\left(R_{T}^{0}\right)\right| \leq C n^{\nu / 3} m^{-1 / 3}+C I^{-1 / 2} .
$$

Proof. From Bishwal (2008, Chapter 8), we have

$$
E\left|\left(\tilde{Y}_{m, T}^{j}-Y_{T}\right) / \sqrt{T}\right|^{2}=O(\Delta T), \quad E\left|\tilde{X}_{m, T}^{j}-X_{T}\right|=O(\Delta T) .
$$

For the large number of Fourier coefficients case, for some $\nu \geq 0$, we have

$$
E\left|\left(\tilde{Y}_{m, T}^{j}-Y_{T}\right) / \sqrt{N}\right|^{2}=O\left(n^{\nu} / m\right), \quad E\left|\tilde{X}_{m, T}^{j}-X_{T}\right|=O\left(n^{\nu} / m\right) .
$$

Consequently, for any $\epsilon>0$, we have

$$
\begin{gathered}
P_{\theta_{0}}^{n, T}\left(\tilde{R}_{n, T}^{0, j}\right) \leq P_{\theta_{0}}^{n, T}\left(\frac{\left(\theta_{1}-\theta_{0}\right)}{2 \sigma\left(\theta_{1}+\theta_{0}\right) \sqrt{T}} X_{T}-Y_{T} / \sqrt{T} \geq \frac{2 \theta_{0} \sigma \Delta \eta \sqrt{T}}{\theta_{1}^{2}-\theta_{0}^{2}}-\epsilon\right) \\
\left.\left.+P_{\theta_{0}}^{n, T}\left|\tilde{Y}_{m, T}^{j}-Y_{T}\right| / \sqrt{T}\right) \geq \epsilon / 2\right)+P_{\theta_{0}}^{n, T}\left(\frac{\left(\theta_{1}-\theta_{0}\right)}{2 \sigma\left(\theta_{1}+\theta_{0}\right) \sqrt{T}}\left|\tilde{X}_{m, T}^{j}-X_{T}\right| \geq \epsilon / 2\right) . \\
P_{\theta_{0}}^{n, T}\left(\frac{\left(\theta_{1}-\theta_{0}\right)}{2 \sigma\left(\theta_{1}+\theta_{0}\right) \sqrt{T}} X_{T}-Y_{T} / \sqrt{T} \geq \frac{2 \theta_{0} \sigma \Delta \eta \sqrt{T}}{\theta_{1}^{2}-\theta_{0}^{2}}-\epsilon\right) \leq P_{\theta_{0}}^{n, T}\left(R_{T}^{0}\right)(1+C \epsilon) .
\end{gathered}
$$

From the above results and Chebyshev inequality, we conclude that

$$
P_{\theta_{0}}^{n, T}\left(\tilde{R}_{n, T}^{0, j}\right) \leq P_{\theta_{0}}^{n, T}\left(\left(R_{T}^{0}\right)(1+C \epsilon)+C \epsilon^{-1} E\left|\tilde{X}_{m, T}^{j}-X_{T}\right| / \sqrt{T}+E\left|\left(\tilde{Y}_{m, T}^{j}-Y_{T}\right) / \sqrt{T}\right|^{2}\right.
$$

Similarly, we have

$$
P_{\theta_{0}}^{n, T}\left(\tilde{R}_{n, T}^{0, j}\right) \geq P_{\theta_{0}}^{n, T}\left(\left(R_{T}^{0}\right)(1-C \epsilon)-C \epsilon^{-1} E\left|\tilde{X}_{m, T}^{j}-X_{T}\right| / \sqrt{T}-E\left|\left(\tilde{Y}_{m, T}^{j}-Y_{T}\right) / \sqrt{T}\right|^{2}\right.
$$

Combining the above two inequalities we obtain

$$
\left|P_{\theta_{0}}^{n, T}\left(\tilde{R}_{n, T}^{0}\right)-P_{\theta_{0}}^{n, T}\left(R_{n, T}^{0}\right)\right| \leq C \epsilon P_{\theta_{0}}^{n, T}\left(R_{T}^{0}\right)+C \epsilon^{-1} E\left|\tilde{X}_{m, T}^{j}-X_{T}\right| / \sqrt{T}+E\left|\left(\tilde{Y}_{m, T}^{j}-Y_{T}\right) / \sqrt{T}\right|^{2}
$$

This implies that

$$
\begin{aligned}
& \left|P_{\theta_{0}}^{n, T}\left(\tilde{R}_{n, T}^{0}\right)-P_{\theta_{0}}^{n, T}\left(R_{n, T}^{0}\right)\right| \leq C(\Delta T)^{1 / 3} . \\
& \operatorname{Var}\left(\frac{\left(\theta_{1}-\theta_{0}\right)}{2 \sigma\left(\theta_{1}+\theta_{0}\right) \sqrt{T}} X_{T}-Y_{T} / \sqrt{T}\right) \leq C
\end{aligned}
$$

From here one can show that

$$
\operatorname{Var}\left(\frac{\left(\theta_{1}-\theta_{0}\right)}{2 \sigma\left(\theta_{1}+\theta_{0}\right) \sqrt{T}} \tilde{X}_{m, T}^{j}-\tilde{Y}_{m, T}^{j} / \sqrt{T}\right)=\operatorname{Var}\left(\frac{\left(\theta_{1}-\theta_{0}\right)}{2 \sigma\left(\theta_{1}+\theta_{0}\right) \sqrt{T}} X_{T}-Y_{T} / \sqrt{T}\right)+O(\Delta T) .
$$

This implies that the Monte Carlo error simulations can be controlled by $I^{-1 / 2}$ uniformly with respect to $T$ and $n$. Therefore we have the following error estimate

$$
\left|\tilde{P}_{\theta_{0}}^{\prime, m, n, T}\left(R_{T}^{0}\right)-P_{\theta_{0}}^{n, T}\left(R_{T}^{0}\right)\right| \leq C(\Delta T)^{1 / 3}+C I^{-1 / 2}
$$


which holds true with high probability (confidence interval for the Monte Carlo estimate) where I is the number of trials of Monte Carlo simulations.

\section{RefERENCES}

[1] R. Adler, P. Muller, B.L. Rozovskii, Stochastic Modeling in Physical Oceonography, Birkhauser, Boston - Berlin. (1996).

[2] S.I. Aihara, Regularized maximum likelihood estimate for an infinite dimensional parameter in stochastic parabolic systems, SIAM J. Cont. Optim. 30 (1992), 745-764.

[3] S.I. Aihara, Maximum likelihood estimate for discontinuous parameter in stochastic hyperbolic systems, Acta Appl. Math. 35 (1994), 131-151.

[4] S.I. Aihara, Identification of a discontinuous parameter in stochastic hyperbolic systems, IFAC Proc.: Syst. Identification, 27 (8) (1994), 191-196.

[5] S.I. Aihara, Identification of a discontinuous parameter in stochastic parabolic systems, Appl. Math. Optim. 37 (1998), 43-69.

[6] S.I. Aihara, A. Bagchi, Parameter identification for stochastic diffusion equations with unknown boundary conditions, Appl. Math. Optim. 17 (1988), 15-36.

[7] S.I. Aihara, A. Bagchi, Infinite dimensional parameter identification for stochastic parabolic systems, Stat. Probab. Lett. 8 (1989), 279-287.

[8] S.I. Aihara, A. Bagchi, Parameter identification for hyperbolic stochastic systems, J. Math. Anal. Appl. 160 (1991), 485-499.

[9] R. Altmayer, T. Bretschneider, J. Janak, M. Reiss, Parameter estimation in a SPDE model for cell repolarization, arXiv:2010.06340 [math.ST]. (2020).

[10] R. Altmayer, I. Cialenko, G. Paseman, Parameter estimation for semilinear SPDes from local measurements, arXiv:2004.14728 [math.ST]. (2020).

[11] K.I. Babenko, On the asymptotic behavior of eigenvelues of linearlized Navier-Stokes equations, Dokl. Akad, Nauk. SSSR, 263 (3) (1982), 521-525.

[12] J.P.N. Bishwal, Bayes and sequential estimation in Hilbert space valued stochastic differential equations, J. Korean Stat. Soc. 28 (1) (1999), 96-108.

[13] J.P.N. Bishwal, The Bernstein-von Mises theorem and spectral asymptotics of Bayes estimators for parabolic SPDEs, J. Aust. Math. Soc. 72 (2) (2002), 289-300.

[14] J.P.N. Bishwal, Rates of weak convergence of the approximate minimum contrast estimators for the discretely observed Ornstein-Uhlenbeck process, Stat. Probab. Lett. 76 (13) (2006), 1397-1409.

[15] J.P.N. Bishwal, Parameter Estimation in Stochastic Differential Equations, Lecture Notes in Mathematics, 1923, Springer-Verlag. (2008a).

[16] J.P.N. Bishwal, Large deviations in testing fractional Ornstein-Uhlenbeck models, Stat. Probab. Lett. 78 (8) (2008b), 953-962.

[17] J.P.N. Bishwal, Uniform rate of weak convergence for the minimum contrast estimator in the Ornstein-Uhlenbeck process, Methodol. Comput. Appl. Probab. 12 (3) (2010), 323-334.

[18] J.P.N. Bishwal, Sieve estimator for fractional stochastic partial differential equation, Ann. Constantin Brancusi-Eng. Ser. 5 (1) (2011a), 9-18.

[19] J.P.N. Bishwal, Minimum contrast estimation in fractional Ornstein-Uhlenbeck process: continuous and discrete sampling, Fract. Calc. Appl. Anal. 14 (3) (2011b), 375-410.

[20] J.P.N. Bishwal, Hypothesis testing for fractional stochastic partial differential equations with applications to neurophysiology and finance, Asian Res. J. Math. 4 (1) (2017), 1-24. 
[21] J.P.N. Bishwal, Benstein-von Mises theorem and small noise Bayesian asymptotics for parabolic stochastic partial differential equations Theory Stoch. Proc. 23 (1) (2018), 6-17.

[22] J.P.N. Bishwal, A. Bose, Rates of convergence of approximate maximum likelihood estimators in the OrnsteinUhlenbeck process, Computers Math. Appl. 42 (1-2) (2001), 23-48.

[23] Borkar, V. and Bagchi, A. (1984)Parameter identification in infinite dimensional linear systems, Stochastics 12, 201-213.

[24] R. Carmona, B.L. Rozovskii, Stochastic Partial Differential Equations: Six Perspectives, American mathematical Society, Rhode-Island. (1999).

[25] I. Cialenko, N. Glatt-Holtz, Parameter estimation for the stochastic Navier-Stokes equation, Stoch. Process. Appl. 121 (2011), 701-724.

[26] I. Cialenco, S. Lototsky, Pospisil, Asymptotic properties of the maximum likelihood estimator for stochastic parabolic equations with assitive fractional Brownian montion, Stoch. Dyn. 9 (2009), 169-185.

[27] I. Cialenko, L. Xu, A note on error estimation for hypothesis testing problems for some linear SPDEs, Stoch. PDE: Anal. Comput. 2 (2014), 408-431.

[28] I. Cialenko, L. Xu, Hypothesis testing for stochastic PDEs driven by additive noise, Stoch. Process. Appl. 125 (2015), 819-866.

[29] I. Cialenko, F. Delgado-Vences, H.J. Kim, Drift estimation for discretely sampled SPDEs, Stoch. PDEs: Anal. Comput. 8 (2020), 895-920.

[30] P. Constantin, C. Foias, Navier-Stokes Equations, Chicago Lectures in Mathematics, University of Chicago Press, Chicago, IL. (1988).

[31] G. Da Prato, J. Zabczyk, Stochastic Equations in Infinite Dimensions, Cambridge University Press, Cambridge, (1992).

[32] F. Frandoli, An Introduction to 3D Stochastic Fluid Dynamics, Lecture Notes in Mathematics 1942, Springer. (2008).

[33] H. Holden, B. ksendal, J. Ube, T. Zhang, Stochastic Partial Differential Equations, Birkhäuser, Boston. (1996).

[34] M. Huebner, A characterization of asymptotic behaviour of maximum likelihood estimators for stochastic PDE's, Math. Meth. Stat. 6 (1997), 395-415.

[35] M. Huebner, Asymptotic properties of the maximum likelihood estimator for stochastic PDEs disturbed by small noise, Stat. Inference Stoch. Proc. 2 (1999), 57-68.

[36] M. Huebner, R.Z. Khasminskii, B.L. Rozovskii, Two examples of parameter estimation for stochastic partial differential equations, InS. Cambanis, J.K. Ghosh, R.L. Karandikar, P.K. Sen (Eds.) Stochastic Processes, Freschrift in honour of G. Kallianpur, Springer-Verlag, Berlin, (1992), 149-160.

[37] M. Huebner, B.L. Rozovskii, On the asymptotic properties of maximum likelihood estimators for parabolic stochastic PDEs, Prob. Theor. Rel. Fields 103 (1995), 143-163.

[38] K. Itô, Foundations of Stochastic Differential Equations in Infinite Dimensional Spaces, CBMS-NSF Regional Conference Series in Applied Mathematics, 49, SIAM, Philadelphia, Pennsylvania. (1984).

[39] G. Kallianpur, R.S. Selukar, Estimation of Hilbert space valued parameter by the method of sieves, In :Statistics and Probability, A R.R.Bahadur Festschrift (Eds.) J.K.Ghosh, S.K.Mitra, K.R.Parthasarathy and B.L.S.Prakasa Rao, Wiley Eastern, (1993), 325-347.

[40] G. Kallianpur, X. Xiong, Infinite Dimensional Stochastic Differential Equations, Lecture Notes-Monograph Series, 26, Institute of Mathematical Statistics, California. (1995).

[41] Y.T. Kim, Parameter estimation for an infinite dimensional stochastic differential equations, J. Korean Stat. Soc. 25 (2) (1996), 161-173.

[42] T. Koski, W. Loges, Asymptotic statistical inference for a stochastic heat flow problem, Stat. Probab. Lett. 3 (1985), 185-189. 
[43] T. Koski, W. Loges, On minimum contrast estimation for Hilbert space valued stochastic differential equations, Stochastics 16, 217-225. functions, Scand. J. Stat. 21 (1986), 421-431.

[44] Yu. N. Linkov, Asymptotic Methods of Statistics for Stochastic Processes, Naukova, Dumka (In Russian). (1993).

[45] R.S. Liptser, A.N. Shiryayev, Theory of Martingales, Kluwer, Dordrecht. (1989).

[46] W. Loges, Girsanov theorem in Hilbert space and an application to the statistics of Hilbert space-valued stochastic differential equations, Stoch. Proc. Appl. 17 (1984), 243-263.

[47] S.V. Lototsky, B. L. Rozovskii, Spectral asymptotics of some functionals arising in statistical inference for SPDEs, Stoch. Process. Appl. 79 (1999), 69-94.

[48] G. Metivier, Valeurs propres d'operateurs definis par la restriction de systemes variationnels a des sous-espaces, J. Math. Pures Appl. 57 (2) (1978), 133-156.

[49] R. Mikulevicius, B.L. Rozovskii, Stochastic Navier-Stokes equations for turbulent flows, SIAM J. Math. Anal. 35 (5) (2004), 1250-1310.

[50] J. Mohapl, Maximum likelihood estimation in linear infinite dimensional models, Commun. Statist. - Stoch. Models 10 (1994), 781-794.

[51] J. Mohapl, On estimation in planar Ornstein-Uhlenbeck process, Comm. Statist.- Stoch. Models 13 (1997), $433-455$.

[52] G. Paseman, W. Stannat, Drift estimation for stochastic reaction diffusion system, Electron. J. Stat. 14 (2020), 547-579.

[53] L. Piterbarg, B.L. Rozovskii, On asymptotic problems of parameter estimation in stochastic PDEsdiscrete time sampling, Math. Methods Stat. 6 (2) (1996), 200-223.

[54] D. Schnoerr, R. Grima, G. Sanguinetti, Cox process representation and inference for stochastic reaction diffusion equation, Nature Commun. 7 (2016), 11729.

[55] K. Tanaka, Distributions of the maximum likelihood and minimum contrast estimators associated with the fractional Ornstein-Uhlenbeck process, Stat. Inference Stoch. Proc. 16 (2013), 173-192.

[56] R. Temam, Navier-Stokes Equations and Nonlinear Functional Analysis, 2nd Ed., In: CMMS-NSF Regional Conference Series in Applied Mathematics, Vol. 66, Society for Industrial and Applied Mathematics (SIAM), Philadelphia, PA. (1995).

[57] R. Temam, Navier-Stokes Equations: Theory and Numerical Analysis, AMS Chelsea Publishing, Providence, RI. Reprint of 1984 Edition. (2001).

[58] A.N. Shiryayev, Probability, Springer-Verlag, New-York. (1996).

[59] J.B. Walsh, An introduction to stochastic partial differential equations, Lecture Notes in Mathematics 1180, 265-439, Springer, Berlin. (1986). 